TOWARD SPATIAL AND SPECTRAL CONTROL OF WAVEGUIDED HIGH-H ARMONIC GENERATION 


\section{Toward spatial and spectral control of waveguided high-harmonic generation}

by

Siew Jean Goh 


\section{Ph.D. graduation committee}

\section{Chairman \& secretary:}

Prof. dr. Ir. J.W.M. Hilgenkamp University of Twente

\section{Promotor:}

Prof. dr. K.-J. Boller

University of Twente

Co-promotor:

Dr. Ing. H.M. J. Bastiaens

University of Twente

Members:

Prof. dr. H. Zacharias

University of Münster

Prof. dr. C. Fallnich

University of Münster \& University of Twente

Prof. dr. J. L. Herek

University of Twente

Prof. dr. F. Bijkerk

University of Twente

Copyright (C) Siew Jean Goh (2015)

Toward spatial and spectral control of waveguided high-harmonic generation

Ph.D. Thesis, Laser Physics and Nonlinear Optics group,

University of Twente, Enschede, The Netherlands

ISBN: 978-90-365-3990-6

DOI: http://dx.doi.org/10.3990/1.9789036539906 


\section{TOWARD SPATIAL AND SPECTRAL CONTROL OF WAVEGUIDED HIGH-HARMONIC GENERATION}

\section{DISSERTATION}

to obtain

the degree of doctor at the University of Twente, on the authority of the rector magnificus,

Prof.dr. H. Brinksma,

on account of the decision of the graduation committee, to be publicly defended

on Wednesday $28^{\text {th }}$ October, 2015 at 1645

by

Siew Jean Goh

born on 1 December 1983

in Kuala Lumpur, Malaysia 
This dissertation has been approved by:

Supervisor: Prof. dr. K.-J.Boller

Co-Supervisor: Dr. Ing. H.M. J. Bastiaens

This research is supported by the Dutch Technology Foundation STW, which is part of the Netherlands Organisation for Scientific Research (NWO) and partly funded by the Ministry of Economic Affairs (project number 10759). 


\section{Contents}

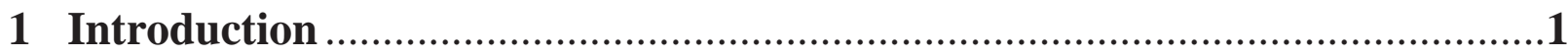

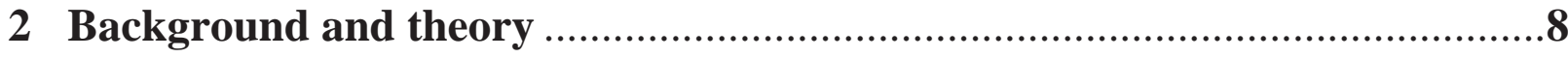

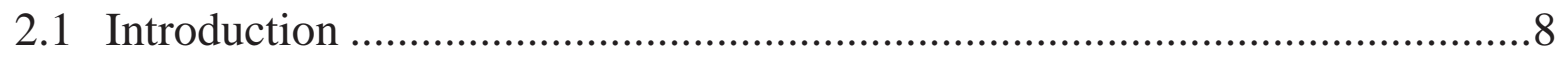

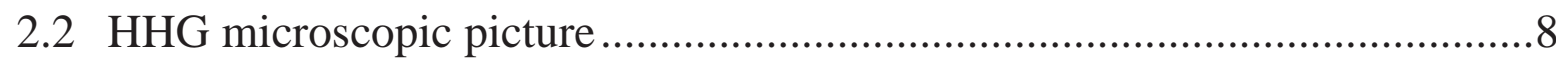

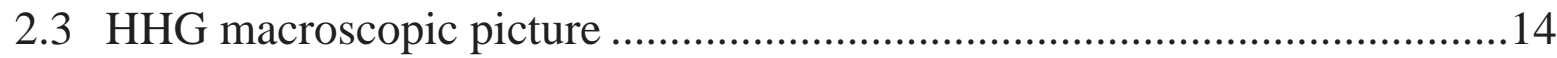

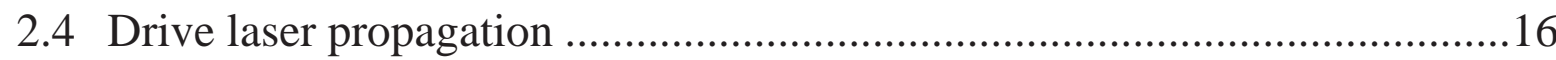

2.4.1 Waveguide propagation.....................................................................16

2.4.2 Nonlinear Kerr effects ....................................................................18

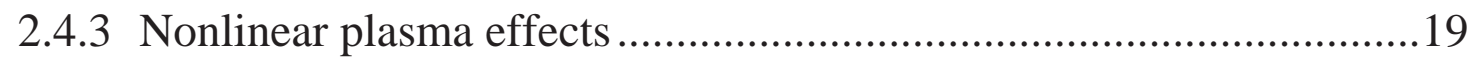

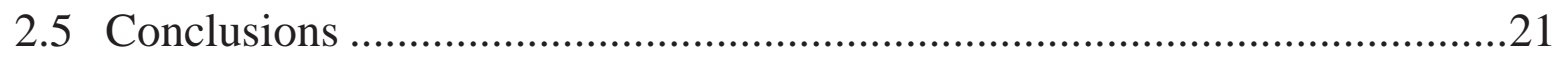

3 Single-shot fluctuations of waveguided high-harmonic generation ...............22

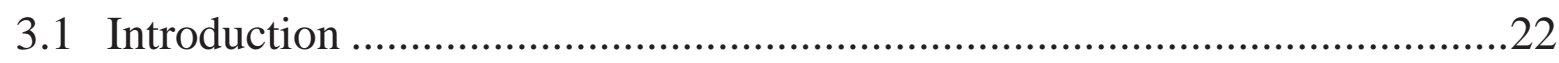

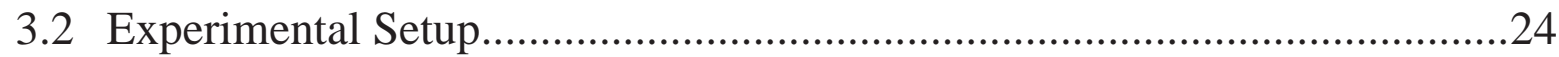

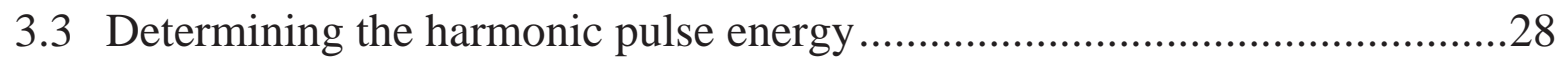

3.4 High-harmonic beam profile, pulse energy and energy jitter ........................29

3.5 Measurement of harmonic beam divergence and pointing stability ..............32

3.6 Correlation measurement of beam parameters between driver laser and $\mathrm{HH}$

3.7 Summary and Conclusions ………………..................................................39

4 Upscaling drive energy and waveguide dimensions for enhanced high

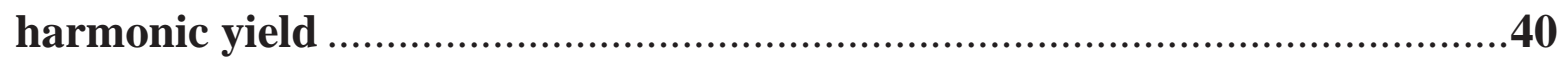

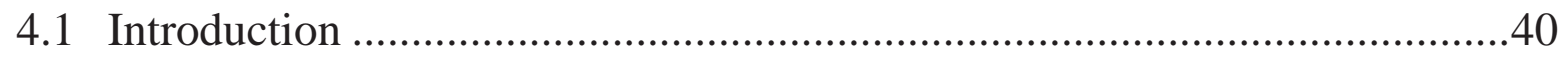

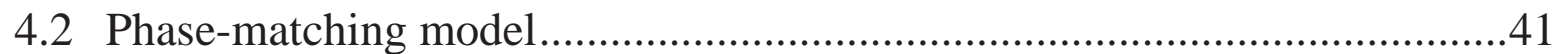

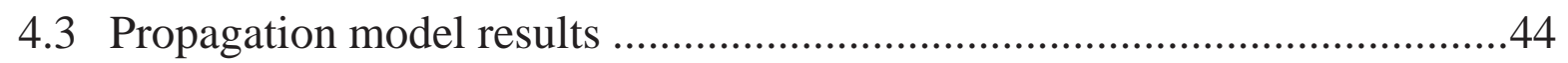

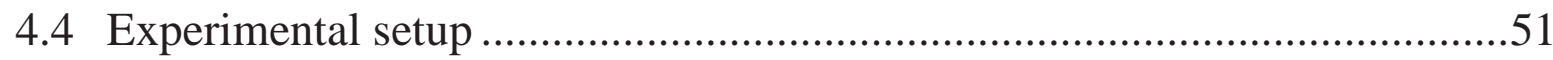




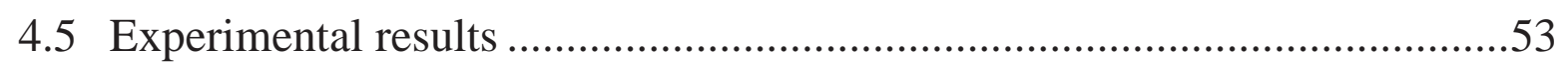

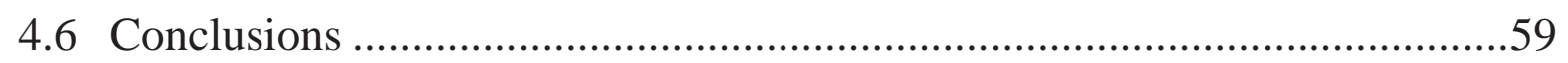

5 Spectral control of high-harmonic generation in a wide-diameter

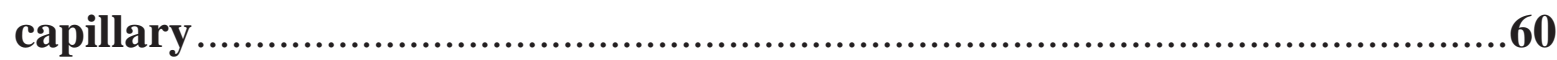

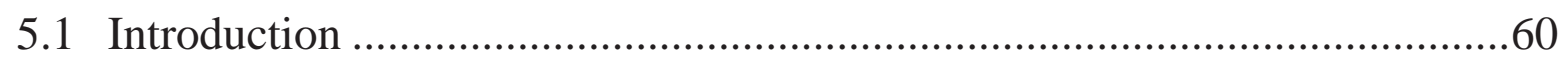

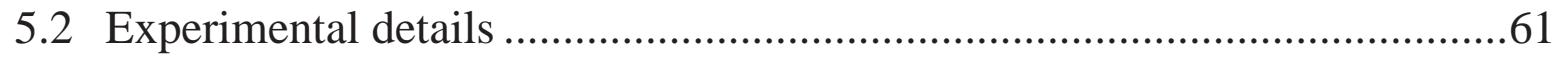

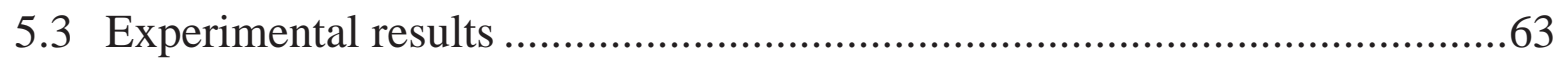

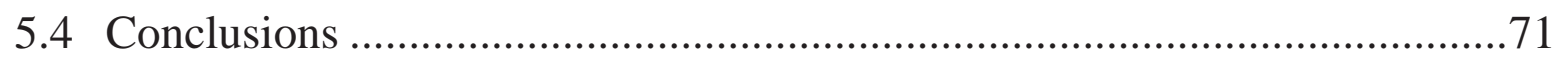

6 Fabrication and characterization of XUV transmission grating....................72

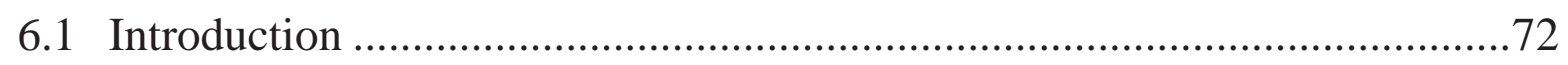

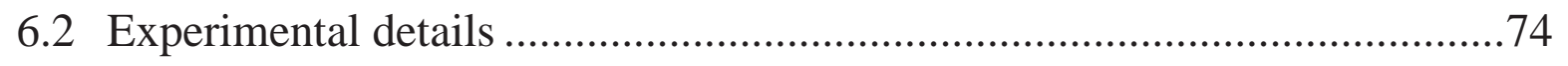

6.2.1 Transmission grating spectrometer .....................................................74

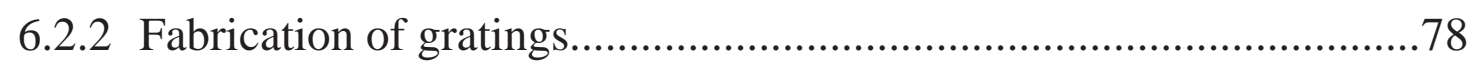

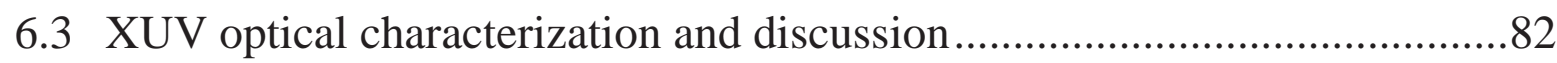

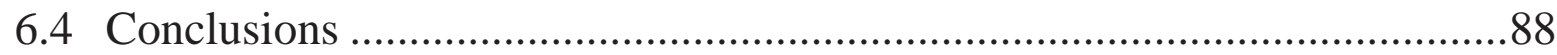

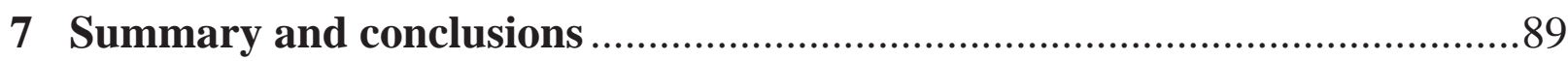

$\begin{array}{lr}\text { Appendix A } & 94\end{array}$

$\begin{array}{lr}\text { References } & 97\end{array}$

$\begin{array}{ll}\text { List of publications } & 106\end{array}$

$\begin{array}{ll}\text { Acknowledgments } & 108\end{array}$ 


\section{1 \\ Introduction}

Since the invention of laser in 1960 [1], the development of lasers covered a huge spectral range including the far-infrared [2, 3], infrared, visible, UV and X-rays [4]. Yet, achieving intense radiation in the wavelength band of extreme ultraviolet (XUV, 100 to $10 \mathrm{~nm}$ ) is still a challenging goal of scientific research for two main reasons. First is the difficulty of lasing at short wavelength as the pump power is inversely proportional to the fourth power of the wavelength [5]. Second is the lack of material suitable for cavity mirrors in the XUV band; therefore amplification is only viable through single pass. There are a number of fundamentally different approaches to generate radiation in the XUV band. For a comparison, Fig.1.1 summarizes the peak spectral brightness (brilliance) [6] as a function of photon energy (wavelength), for the laser-produced plasma sources (LPP [7]), synchrotrons (ALS [8] and ESRF [9]), free-electron lasers (FLASH [10], XFEL[11] and LCLS [12]) and via nonlinear optical generation, i.e., high-order harmonic generation. From Fig.1.1, it can be seen that the free electron lasers deliver the highest brightness values compared to other sources with wavelength ranging from 124 to $0.124 \mathrm{~nm}$. Note that (peak) brightness and available wavelength range alone do not determine the suitability as a light source for a particular application, also other properties such as bandwidths, spatial and temporal coherence, pulse stability and repetition rates play a role.

Laser-produced plasma sources employ a pulsed laser beam with focused intensities of $10^{16}$ to $10^{18} \mathrm{~W} / \mathrm{cm}^{2}$ onto a solid target. The solid target is evaporated and ionized which generates plasma. The excited ions then decay and emit bright XUV radiation either via bremsstrahlung (free-free transition) [13, 14], radiative recombination (bound-free transition) [15] or line emission (bound-bound transition) from population inversion on particular transitions $[14,16]$. The LPP emission can have broad spectral background due to the thermal emission and can sometimes exhibit characteristic lines as well, i.e., strong LPP emission lines at $13 \mathrm{~nm}$ from tin droplets [17]. The LPP using tin droplets is considered as a suitable candidate for XUV lithography because overall setup can be realized with a compact format and appreciable average powers with repetition rates up to several tens of $\mathrm{kHz}$ [17]. The LPP radiation is spontaneous and emitted in $2 \pi$ solid angle where collecting the radiation is difficult and optics can be contaminated with ablated material. However many promising efforts have been undertaken to solve these issues [18-21]. 
Synchrotron sources are based on circular electron storage rings where accelerated electrons are injected with relativistic speed and spontaneous light is produced by electrons passing through bending magnets [22]. Using undulators (wigglers) enhanced spontaneous radiation is obtained in certain spectral regions, while the relativistic velocity of the electrons gives rise to a strongly collimated radiation into the forward direction. The central wavelength of these spectral regions is set by the wiggler period and tuning is obtained by varying the gap between the magnets. Although undulators and wigglers produce light with a strongly reduced bandwidth (compared to light from bending magnets), in most cases a monochromator is used to spectrally narrow the light even further. Based on the short duration of the generated optical pulse caused by the electron beam traveling in the form of short bunches, typically with durations shorter than a nanosecond, and due to high average brightness, synchrotron radiation is well-suited for timeresolved imaging $[23,24]$ and spectroscopy [24].

Wavelength $(\mathrm{nm})$

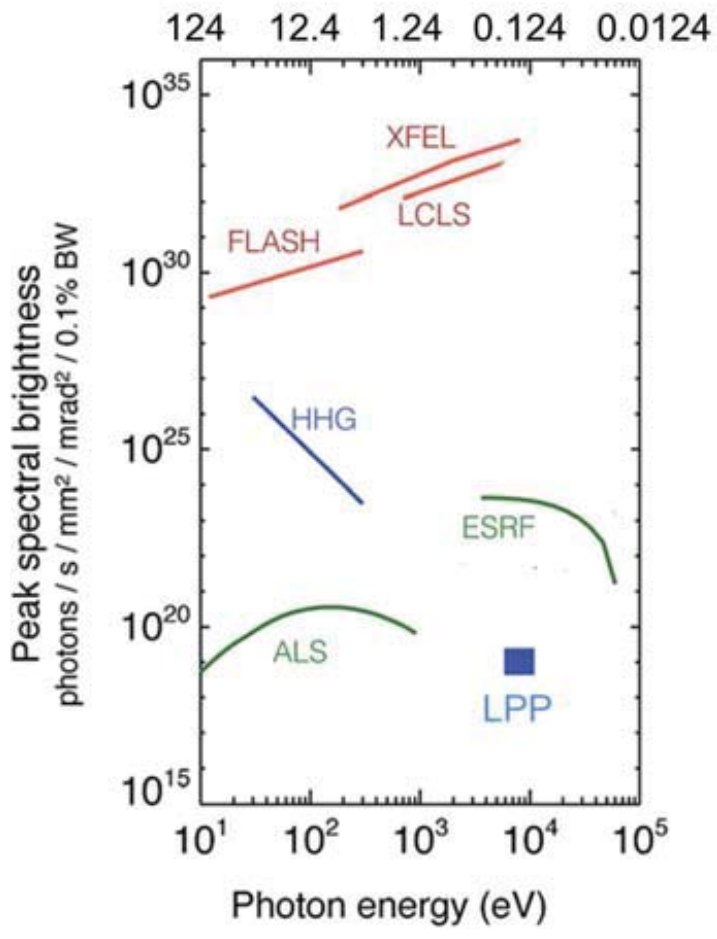

Figure 1.1: Comparison of the peak spectral brightness as a function of photon energy (wavelength) of XUV sources employing various fundamentally different approaches for generation of the radiation. (figure adapted from [6].)

Free-electron lasers (FEL) are typically linear-accelerator based sources, again based on a beam of relativistic electrons. Within the electron pulse, electrons will bunch together under influence of the magnetic field of the undulator and their own radiation, leading to spatially and spectrally coherent output. The gain medium 
consists of free electrons and FEL radiation can be generated with extreme brightness. The frequency of the FEL radiation can be continuously tuned via the electron velocity and gap of the undulator (i.e., the on-axis strength of the periodic magnetic field). FEL radiation can be generated from microwaves to the $\mathrm{x}$-ray regime, though in the XUV and X-ray region, where mirrors are not readily available, high peak currents and long undulators are required to saturate the laser in a single pass. The latter process is known as Self Amplified Spontaneous Emission (SASE). The peak brilliance is currently ten orders of magnitude greater than that of synchrotrons and the pulse duration is much shorter as well, from a few to $100 \mathrm{fs}$.

The high brilliance along with temporal and spatial resolution at atomic time and length scales have opened up for new types of time-resolved experiments such as coherent diffractive imaging [25], nonlinear atomic physics [26], extreme nonlinear optics [27] and gas diffraction [28] experiments carried out at LCLS. However, since the electron bunches form spontaneously in SASE based FELs, the output beam has limited temporal coherence. Specifically, the output pulses vary noticeably in their spectral content and temporal structure, also showing undesired fluctuations of the peak power. To solve this issue, a widely pursued approach is to use injection seeding [29]. Here, radiation is injected that is much weaker but is coherent shot-toshot and the FEL is used to amplify the injected input or a higher harmonic of it [29, 30]. For such injection, the FEL amplification is highest if the wavelength of both the seed and the FEL are the same. High-harmonic $(\mathrm{HH})$ generation as discussed next, being the subject of the investigation in this thesis is currently the most promising way for direct seeding at XUV wavelengths, provided that the generated $\mathrm{HH}$ themselves is sufficiently stable from shot to shot and can be tuned for overlapping the gain profile and match the temporal amplification window of an FEL to be injection seeded. Even when the FEL lases at a harmonic of the seed, using $\mathrm{HH}$ may be beneficial as it would allow the generation of shorter wavelengths.

High-harmonic generation (HHG) described in this thesis uses a pulsed laser beam with an extremely high peak intensity of $10^{14} \mathrm{~W} / \mathrm{cm}^{2}$ which is typically focused into a gaseous target as shown in Fig.1.2. The gas medium then generates light with frequencies that are integer multiples of the drive laser frequency, thereby reaching the XUV regime. HHG was first reported by McPherson et.al [31] in 1987 and then by Ferry et.al [32] in 1988 where they both observe what is called a spectral plateau that consists of equally intense harmonics of high order followed by a sharp cutoff at some maximum light frequency. The observed spectral distribution with a plateau and cutoff cannot be explained with standard perturbation theory because in that approximation the harmonic intensity decreases with increasing harmonic order [33]. 


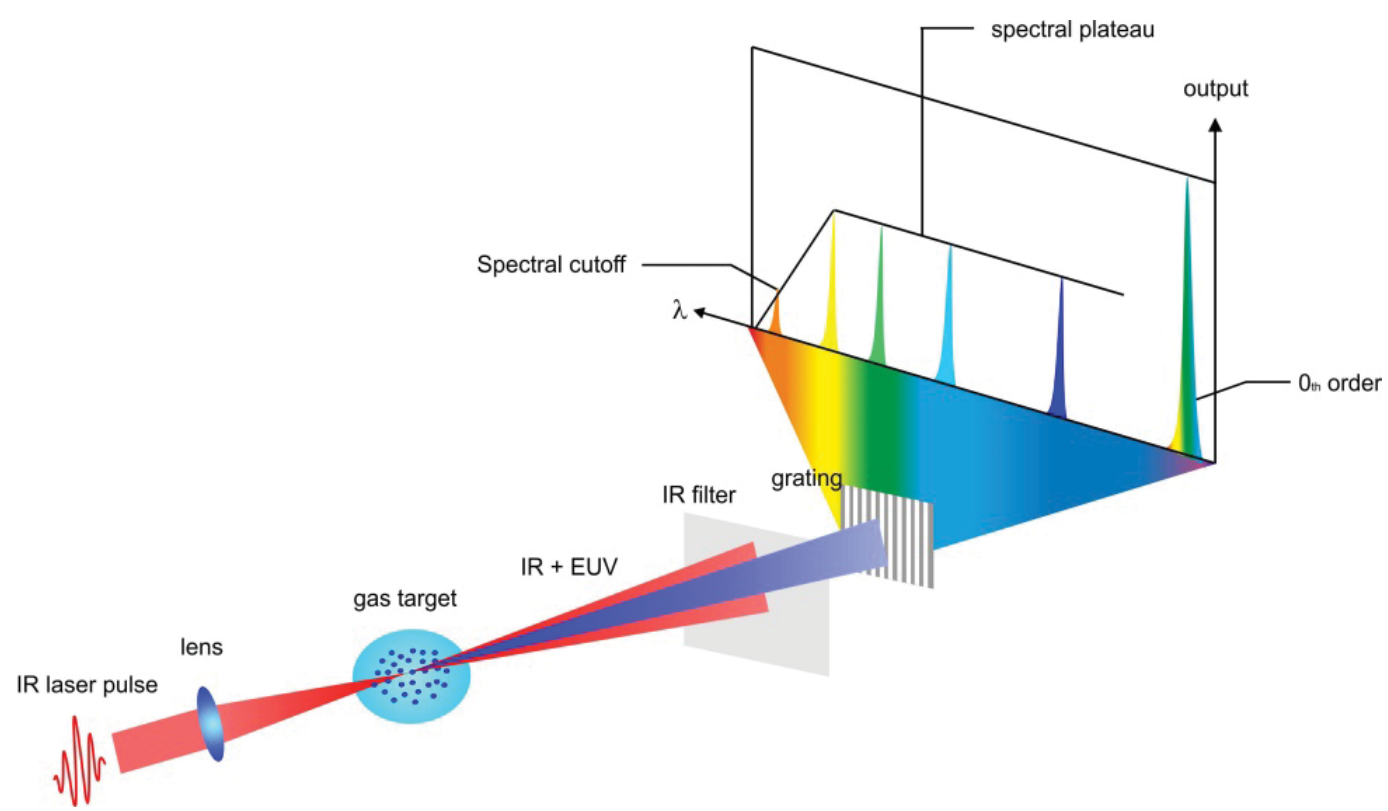

Figure 1.2: Illustration of a table-top setup for high-harmonic generation based on a gaseous target where the generated XUV radiation is spectrally resolved via grating.

It wasn't until 1993 that Corkum et. al present a semi-classical theory [34] which predicts the plateau and cutoff observed in the experiments. When looking at a single atom, their semi-classical theory approximates HHG processes based on three separate steps as shown in Fig.1.3. The first is tunnel ionization of an electron under the influence of the strong applied field of the drive laser. The second step is acceleration in the laser field, away from the parent ion first and later when the laser field changes sign, the electron accelerated back to the parent ion, where the electron arrives with significantly kinetic energy. The third step is the collision (interaction) with the parent ion leading, with a certain probability, to recombination. In this step, a burst of XUV radiation is emitted that carries away the total energy of the electron as XUV photons.
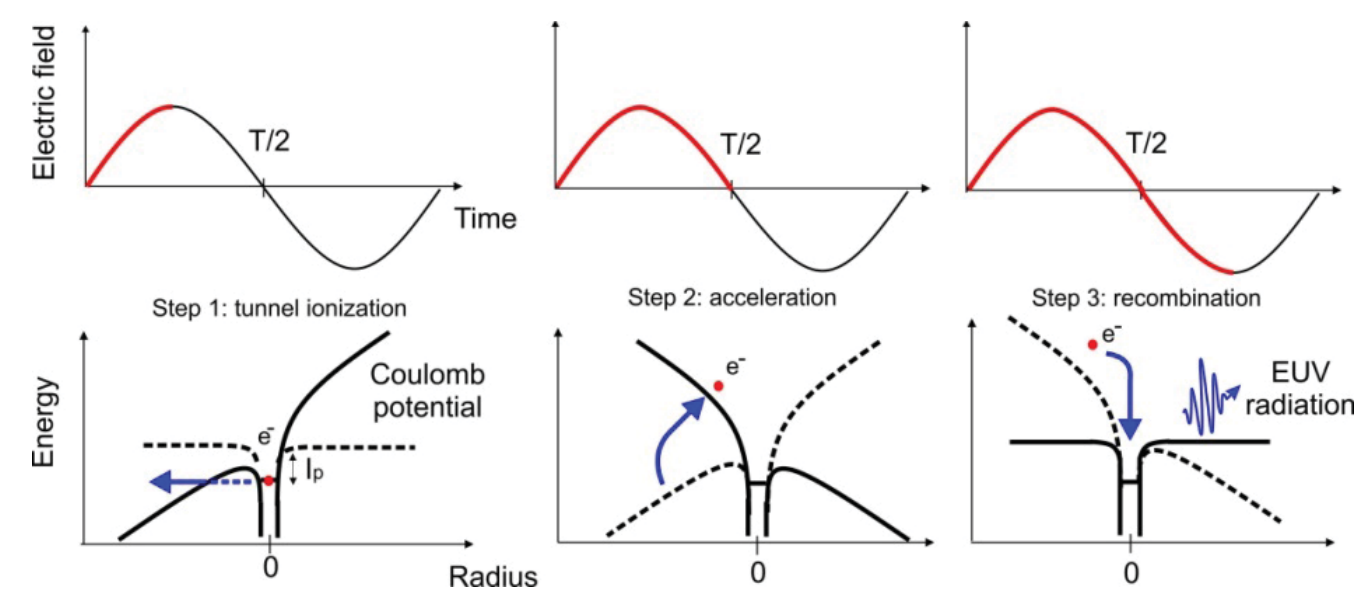

Figure 1.3: Illustration of the high-harmonic generation based on the three-step model. The blue arrows illustrate the trajectories of the ionized electron. 
Already for a single atom the described dynamics in the three-step model is of significant complexity, making predictions of the absolute output rather difficult. A second type of complexity arises because the process typically involves an active volume of the gas medium, such that the mutual coherence of the atomic emitters and superposition of their contributions have to be taken into account via phase matching considerations. Finally, the drive laser pulse propagating through the gas may undergo modifications via associated nonlinear optical processes, rendering pulse-to-pulse fluctuating excitation conditions. In view of these conditions a comprehensive modeling of $\mathrm{HHG}$ is required for an accurate description of this process. For a qualitative description, focused only on certain parts of the overall process, simplified models can be used to obtain an improved understanding of the HHG process. More details on the three-step model will be discussed later in this thesis.

When looking at the temporal structure of the generated harmonics, the HHG radiation consists of a burst or regular train of attosecond pulse depending on whether there is a proper phasing between the harmonics [35, 36]. This unprecedented temporal resolution has enabled new research fields such as attosecond science [37]. Examples are the generation of isolated attosecond pulse via few cycles drive laser pulse [38] or polarization gating [39]. Attoscience has opened up or is currently exploring many new applications, e.g., probing electron dynamics [40] and molecular dynamics [41].

In comparison with SASE FELs which generate coherent beams only in the spatial domain, HHG gives fully coherent beams in both the temporal and spatial domains [42, 43]. HHG can comprise of a table-top setup which allows for experiments such as coherent diffraction imaging [44], HHG spectroscopy [45, 46], HHG interferometry [47] to be carried out in a standard optical laboratory. This is of importance for unrestricted access, other than in large-scale facilities. The excellent coherence in both spatial and temporal domain and the possibility of wavelength tunability $[48,49]$ make the HHG source a promising candidate for direct seeding of XUV FELs in order to achieve fully coherent FEL output [50].

Nevertheless, there are also fundamental restrictions to HHG. A known issue with $\mathrm{HHG}$ is the relatively low conversion efficiency, typically lower than $10^{-7}$ and record-values reaching at most the $10^{-5}$ level. The typical type of laser used for driving HHG is a Titanium Sapphire oscillator amplifier system with 1 to $10 \mathrm{Watt}$ average output power, which leads to a pulse energy of up to $10 \mathrm{~mJ}$ in a system with $1 \mathrm{kHz}$ repetition rate. To increase the average photon flux in $\mathrm{HHG}$, lasers with higher repetition have been employed making use of generation in a drive laser resonant cavity scheme [51] which benefits applications such as HHG spectroscopy with improved signal to noise ratio. However, for applications such as single-shot diffractive imaging [52] or seeding of FELs, an increase of pulse energy is required instead of an increased average photon flux. With this goal, one approach to scale up the pulse energy while maintaining phase matching is to use loose focusing which 
requires, however, relatively high pulse energies, in the order of $50 \mathrm{~mJ}[52,53]$. Another approach is make use of waveguiding of the drive laser pulses in a capillary that is filled with a gas to provide a longer interaction length [54]. The optimization of the phase-matching condition for such systems has been investigated extensively $[55,56]$. Due to a large set of experimental parameters, even today, the optimization conditions are still being researched [57].

In view of the described open questions and associated potential, the goal of this thesis is to investigate and characterize the spatial and spectral properties of XUV radiation based on high-harmonic generation in a gas-filled capillary waveguides as a function of multiple experimental parameters. In the next chapter we recall the essentials of the theoretical background required for understanding high-harmonic generation as investigated here.

In geometries based on free propagation of the drive laser beam, i.e., in gas cells and gas jets, fluctuations in HHG have been characterized extensively. For applications of HHG it is of central importance to characterize these fluctuations and distortions, such as for increasing the measurement precision for absolute, nonlinear ionization cross sections [58, 59] and injection seeding at free-electron laser facilities for improving the coherence and the shot-to-shot stability of the laser output [60-62]. In contrast to free propagation, one might expect a lower degree of output fluctuations and a higher degree of spatial coherence, when controlling the drive laser propagation through the gas sample via waveguiding. To our knowledge, there is no characterization or quantification of shot-to-shot (single-pulse) fluctuations or shot-to-shot output beam deformations in waveguided HHG. Changes of the beam direction and shape have only been addressed by averaging over a large number of pulses [63]. In Chapter 3, we present the first shot-to-shot fluctuation of high-harmonic beams generated in a capillary, having a typical diameter of $150 \mu \mathrm{m}$ and typical drive laser pulse energies of hundreds $\mu \mathrm{J}$.

As mentioned before, a known issue with HHG is the relatively low conversion efficiency. For increasing the output, it seems straightforward to apply drive pulses with higher energy. However, HHG in capillaries suffers from limitations such as ionization-induced phase mismatching and the drive laser propagation becomes subject to complicated nonlinear propagation effects. A promising way to circumvent such limitations would be HHG in a capillary with significantly increased cross section, such that mJ-levels of drive laser pulse energies can be applied. In Chapter 4, we theoretically and experimentally investigate the scale up of the high-harmonic output based on this approach. First we introduce a simple theoretical model which potentially can predict the build-up of high-harmonic energy in an Ar-filled capillary over a wider range of parameters, specifically the drive laser pulse energy, the gas pressure, the capillary diameter and the interaction length. Later on, we characterize the high-harmonics generated in a wide-diameter capillary $(508 \mu \mathrm{m})$ using elevated drive energies in the range of several $\mathrm{mJ}$ which is beyond what can be applied to standard (150 $\mu \mathrm{m}$ diameter) capillaries. 
In a wide capillary where the waveguiding induced dispersion is weaker, phase matching occurs at lower gas pressure. This might impose limitations on the spectral control via drive laser shaping because such technique involves a critical timing and sizing of ionization-induced blue shift of the drive laser wavelength [48]. These mechanisms imply that with a wide capillary a higher harmonic output might be obtained while it remains open to what an extent the harmonic output can be tuned via drive laser pulse shaping. It is important to perform an experimental investigation of the spectral control of $\mathrm{HHG}$ in wide-diameter capillaries for identifying the effectiveness of drive laser pulse shaping. Therefore, in Chapter 5, we investigate the wavelength tuning of the HHG in a wide capillary as a function of the gas pressure and the chirp of the drive laser pulses.

In Chapter 6, we demonstrate the advantage of HHG for the characterization of nanostructures. We apply the high-harmonic radiation for characterization of freestanding, high-line-density gratings with up to 10,000 lines per $\mathrm{mm}$ (100 nm grating period) utilized for an XUV spectrometer. We quantify the relative strength of the second order diffraction which depends on imperfections in fabrication that might lead to, e.g., an asymmetric space-to-period ratio. We evaluate the spectral resolution that can be achieved from the grating utilizing the unique features of XUV sources based on HHG, namely, the well-separated odd harmonics and the narrow-bandwidth of the individual harmonic order. Finally, we summarize and conclude in Chapter 7 the major experimental results and findings. 


\section{2 \\ Background and theory}

\subsection{Introduction}

This chapter reviews the background and theory of High-harmonic Generation $(H H G)$ essential to understand the characteristics of the coherent soft-X-ray source presented in this thesis. $H H G$ is a highly nonlinear optical process driven by an intense laser pulse (infrared) that interacts with matter, most commonly with a gaseous medium. This process generates light with frequencies that are integer multiples of the drive laser frequency, thereby reaching the soft-x-ray regime.

We begin in Section 2.2 with the microscopic picture of HHG, described by the three-step model which considers the effects of an intense laser pulse on a single atom. With the model, some features of the HHG spectrum can be well explained. However, in practice, HHG takes place in a macroscopic medium made of many atoms where certain additional conditions have to be fulfilled for observing the output in the form of a beam. This is why we look further into the macroscopic picture of HHG in Section 2.3, where phase-matching plays a central role. More specifically, the HHG flux depends strongly on phase-matching, a condition where the harmonics of all atoms are emitted with proper phasing as the drive laser propagates through the gaseous medium. When phase-matching is fulfilled, the harmonics of all the driven atoms interfere constructively and the HHG flux can build up along the interaction length. Here, we discuss the major contributions to phase-mismatch between the $\mathrm{HH}$ and the drive laser. Lastly in Section 2.4, we will discuss the propagation effects of the drive laser within a capillary filled with gas. Drive laser can propagate in different waveguide modes inside a capillary depending on the focusing condition. Also, depending on the intensity and gas pressure, the drive laser propagation within the capillary can become dependent on nonlinear effects, as described in Section 2.4.

\subsection{HHG microscopic picture}

The microscopic picture of HHG process can be described by a semi-classical model developed by Corkum et.al [34], generally referred as the three-step model. This model illustrates the single atom response to a strong electric field via three steps, I. Ionization of an electron, II. Propagation of the ionized electron and III. 
Recombination of the ionized electron with its parent ion, upon which highharmonic radiation is emitted.

\section{Ionization}

When an ultrashort laser pulse interacts with an atom, a bound electron can be set free from the atom and this process is called ionization. As a function of electric field strength or intensity, one can classify the ionization into different regimes which are named (i) multiphoton ionization, (ii) tunneling ionization and (iii) barrier suppression ionization. An appropriate way to express the accordingly required intensities is via the so-called Keldysh parameter [64], which is defined as

$$
\Gamma=\sqrt{\frac{\mathrm{I}_{\mathrm{p}}}{2 \mathrm{U}_{\mathrm{p}}}},
$$

where $I_{p}$ is the ionization potential of the atom and $U_{p}$ is the ponderomotive energy. The ponderomotive energy, $U_{p}$, is the cycle average quiver energy (kinetic energy) of an electron in an oscillating field, which is given by

$$
\mathrm{U}_{\mathrm{p}}[\mathrm{eV}]=\frac{\mathrm{e}^{2} \mathrm{E}_{0}^{2}}{4 \mathrm{~m}_{\mathrm{e}} \omega^{2}} \approx 9.3 \times 10^{-14} \mathrm{I}\left[\frac{\mathrm{W}}{\mathrm{cm}^{2}}\right] \lambda^{2}[\mu \mathrm{m}],
$$

where $e$ is the electron charge, $E_{0}$ is the drive laser electric field amplitude, $\omega$ is the frequency of the drive laser and $m_{e}$ is the electron mass. From Eq. 2.2, it can see that $U_{p}$ is proportional to the laser intensity and to the square of the laser wavelength. In the regime of comparably lower laser intensities, where $\Gamma>>1$, multiphotonionization (MPI) dominates and the absorption of multiple photons by the atom is required for the electron to bridge the gap between the ground state and the ionization potential. In this regime, perturbation theory can be used to illustrate that the intensity of the generated harmonics decrease with increasing harmonic order. When the laser intensity is higher such that $\Gamma<<1$, the ionization enters a regime where tunneling is dominating. In this regime, the electric field is strong enough to distort the atomic Coulomb potential somewhat and a valence electron may tunnelionize. The laser intensity required is in the range of $10^{14} \mathrm{~W} / \mathrm{cm}^{2}$ for tunnel ionization of a neutral gas atom. If the HHG process takes place in this regime, the emissions no longer follow the laws of perturbation theory, instead a plateau [65, 66] is observed as shown in Fig. 2.1. In the plateau, the intensities of the emission remain constant for increasing harmonic orders until the so-called cutoff where the intensities drop significantly. 


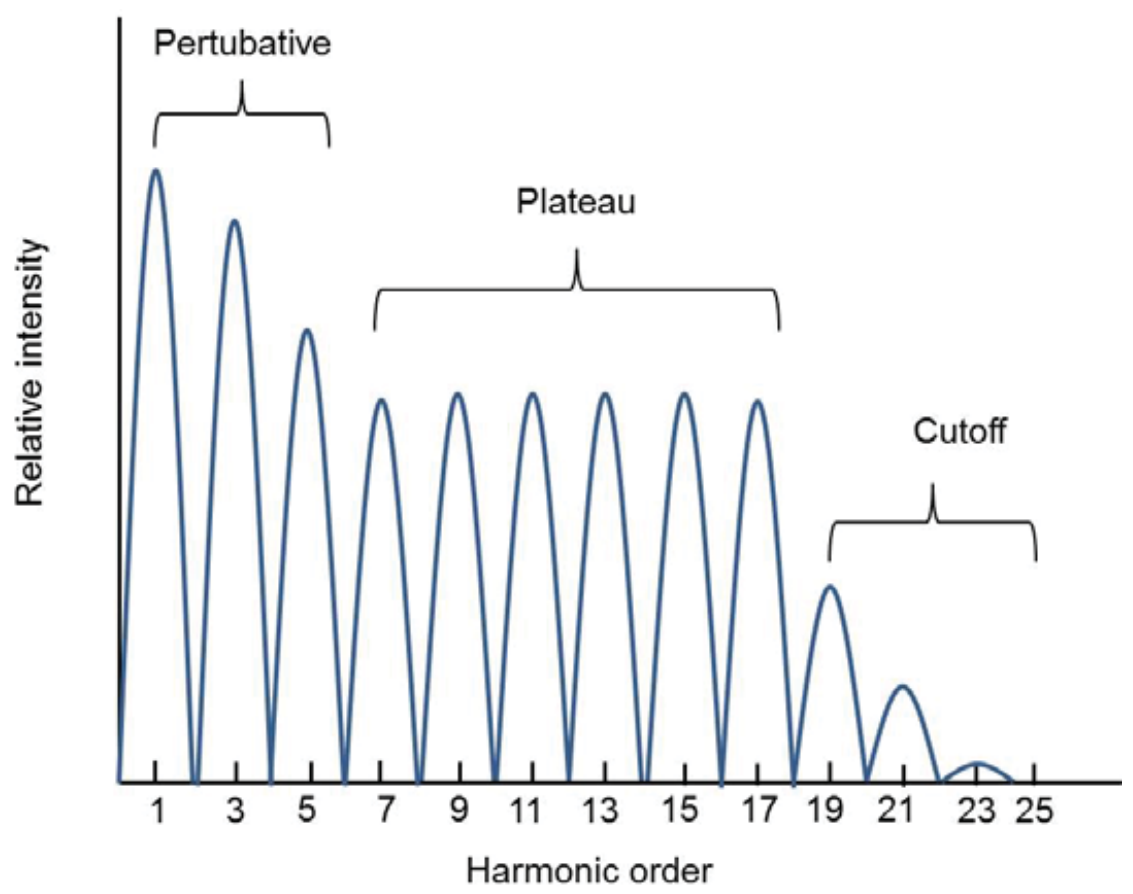

Figure 2.1: Typical spectrum of HHG which can be divided into three regimes: the perturbative region at low orders, the plateau at intermediate orders and cut-off at the highest order.

For the HHG experiments described this thesis, the drive peak intensities are well within this regime. Hence, tunnel ionization is the dominant mechanism. The tunnel ionization rates, $\psi_{A D K}(t)$, can be approximated by a model developed by Ammosov, Delone and Krainov (ADK model), [67], expressed as

$$
\psi_{\mathrm{ADK}}(\mathrm{t})=\omega_{\mathrm{p}}\left|\mathrm{C}_{\mathrm{n}} *\right|^{2}\left(4 \omega_{\mathrm{p}} / \omega_{\mathrm{t}}\right)^{2 \mathrm{n}^{*}-1} \exp \left(-\frac{4 \omega_{\mathrm{p}}}{3 \omega_{\mathrm{t}}}\right) .
$$

Where

$$
\begin{gathered}
\omega_{\mathrm{p}}=\mathrm{I}_{\mathrm{p}} / \hbar \\
\omega_{\mathrm{t}}=\mathrm{eE}_{\mathrm{d}}(\mathrm{t}) /\left(2 \mathrm{~m}_{\mathrm{e}} \mathrm{I}_{\mathrm{p}}\right)^{1 / 2} \\
\left|\mathrm{C}_{\mathrm{n}} *\right|^{2}=2^{2 \mathrm{n} *}\left[\mathrm{n}^{*} \Gamma\left(\mathrm{n}^{*}+1\right) \Gamma\left(\mathrm{n}^{*}\right)\right]^{-1} \\
\mathrm{n}^{*}=\mathrm{Z}\left(\mathrm{I}_{\mathrm{p} /} \mathrm{I}_{\mathrm{h}}\right)^{1 / 2}
\end{gathered}
$$

Here, $I_{p}$ is again the ionization potential of the considered atom or ion in the initial state, $I_{h}$ is the ionization potential of the hydrogen atom, $Z$ is the charge of the ion 
after ionization, $\Gamma$ is the Keldysh parameter and $E_{d}$ is the modulus of the instantaneous electric field of the drive laser pulse. Eq. 2.4 assumes ionization from the ground state of the atom. From the ionization rate, $\psi(t)$, the fraction of free electrons, $\eta(t)=N_{e} / N_{0}$, with $N_{0}$ the initial atom density and $N_{e}$ the electron density, can be calculated from integration of the ionization rate as

$$
\eta(\mathrm{t})=1-\exp \left(-\int_{-\infty}^{\mathrm{t}} \psi_{\mathrm{ADK}}\left(\mathrm{t}^{\prime}\right) \mathrm{dt}^{\prime}\right)
$$

Note that $1-\eta(\mathrm{t})$ is equal to the ionization probability which is important for phase matching considerations as presented in Section 2.3. Finally, when the laser intensity is even higher than in the tunnel regime, the field is strong enough to largely suppress the Coulomb potential barrier. When this happens, the electron can leave the Coulomb binding without tunneling. This process is termed as the barrier suppression ionization (BSI). The intensity threshold for BSI is given by [68]

$$
\mathrm{I}_{\mathrm{c}}=4 \times 10^{9}\left(\frac{\mathrm{I}_{\mathrm{p}}^{4}}{\mathrm{Z}^{2}}\right)
$$

where again $Z$ is the charge of the ion after ionization and $I_{p}$ is the ionization potential of the atom or ion in the initial state in $\mathrm{eV}$.

\section{Second step: Electron propagation}

The propagation of the ionized electron under the influence of a sinusoidal electric field, $E_{d}(t)=E_{0} \cos (\omega t)$, neglecting the potential of the ion core can be described in Newton law as

$$
\mathrm{m}_{\mathrm{e}} \mathrm{a}(\mathrm{t})=\mathrm{eE}_{\mathrm{d}}(\mathrm{t})=\mathrm{eE}_{0} \cos (\omega \mathrm{t})
$$

where $a$ is the acceleration, $E_{0}$ is the amplitude of the electric field and $\omega$ its angular frequency. The position of the electron after ionization can then derived from eq.2.10 as follows:

$$
x\left(t, t_{0}\right)=\iint_{t_{0}}^{t} e E_{0} a(t)=\frac{e E_{0}}{m_{e} \omega^{2}}\left[\cos \left(\omega t_{0}\right)-\cos (\omega t)-\left(\omega t-\omega t_{0}\right) \sin \left(\omega t_{0}\right)\right]
$$

where $t_{0}$ denotes the time of ionization. Because ionization is a quantum process, the exact time of ionization cannot be predicted but only the ensemble averaged value. The individual electron, depending on the actual time of ionization, $\mathrm{t}_{0}$, and the 
associated phase, $\phi_{0}=\omega t_{0}$, of the laser field at that time, can take different types of trajectories. This is shown in Figure 2.2 where the dashed line indicates the phase of the laser field, $\phi=\omega t$. Electron are most likely ionized around the peak of the electric field $\left(\phi=0^{\circ}\right)$ but they return to the ion with zero kinetic energy. Most electrons are produced at unfavorable laser phase $\left(-90^{\circ}<\phi<-0^{\circ}\right)$ because these trajectories never return to the ion. In the plateau regime of high-harmonic emission, typically two main contributions from the electron trajectories form the harmonic emission. One contribution is from the so-called short trajectories $\left(18^{\circ}<\phi<90^{\circ}\right)$ which correspond to a short return time. And the other contribution is from the long trajectories $\left(0^{\circ}<\phi\right.$ $<18^{\circ}$ ), which correspond to a long return time, in the course of one optical period. The long trajectory leads to a strong intensity dependence of the harmonic phase. The light generated at the shortest wavelengths near cutoff belongs to electron trajectories that have the highest velocity and highest kinetic energy upon return to the ion generated by electrons that are ionized near $\phi=18^{\circ}$.

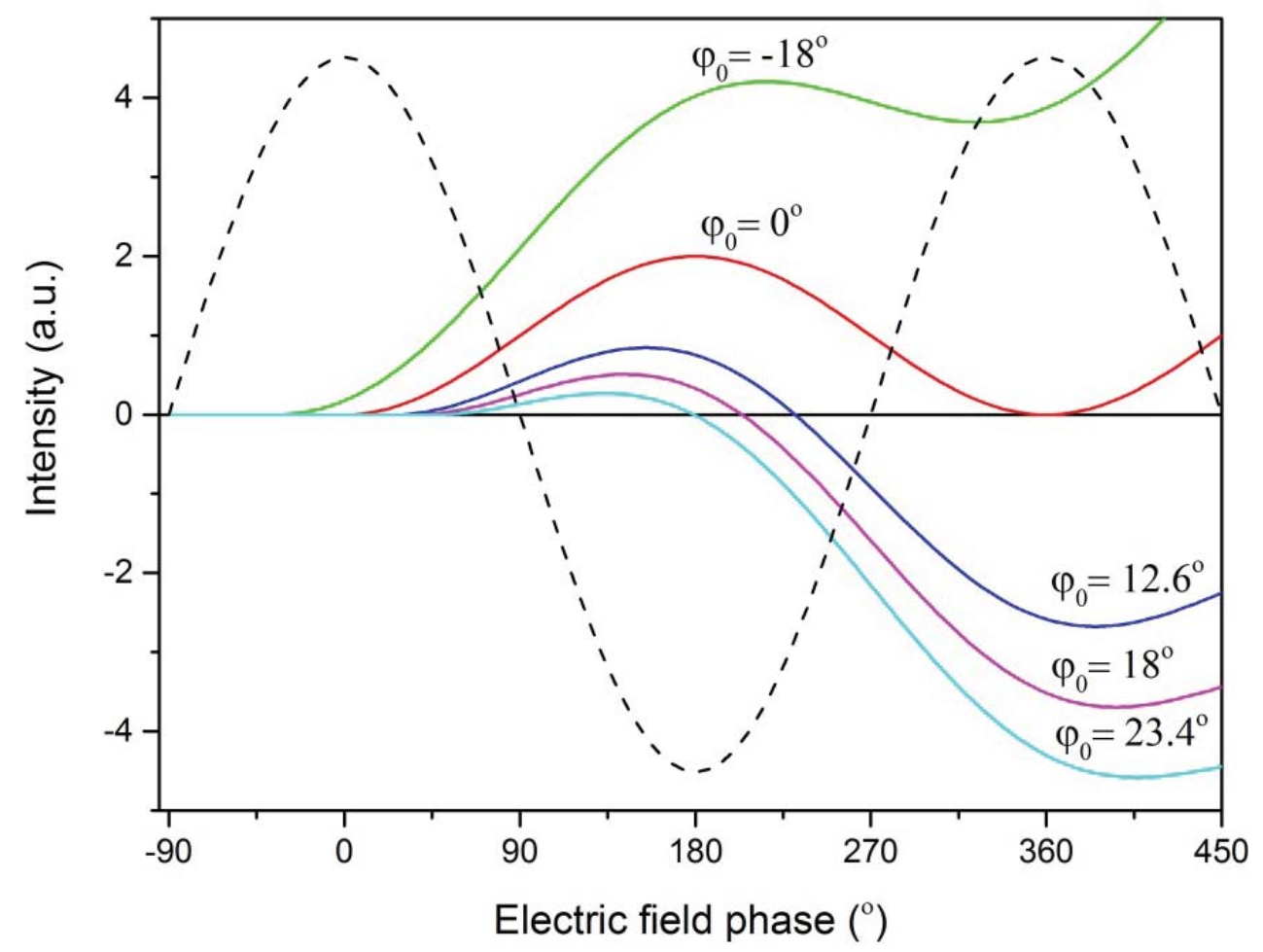

Figure 2.2: The position of an electron as a function of the phase of electrical field for different trajectories. These electron trajectories are represented by their own ionization phase, $\phi_{0}$, with respect to the electric field (black dashed line), indicating electron which never return to its ion (green), return with zero kinetic energy (red), return with the cutoff energy (magenta), return with the same energy via a short trajectory (light blue) and long trajectory (blue). 


\section{Third step: Recombination}

When the electron returns to its parent ion, it may recombine with the ion and transit back to its ground state which leads to a short burst of radiation. The maximum energy of the radiated photons can be calculated as the sum of the ionization potential and the additional kinetic energy gathered by the electron along the path from the turning point by the trajectory back to the atom. As was described before, the kinetic energy depends on the ionization phase, $\phi_{0}$ of the electron and can be calculated as a function of $\phi_{0}$ by solving Eq. 2.11 for $x\left(\phi_{0}, t\right)=0$. The maximum kinetic energy is obtained for a phase of 18 degree as shown in Fig. 2.3 and takes the value of $3.17 \mathrm{U}_{\mathrm{p}}$. The energy of the emitted photon is equal to sum of the kinetic energy and ionization potential. Therefore, the maximum photon energy corresponding to the so-called cutoff energy, $\mathrm{E}_{\mathrm{c}}$ can be expressed as

$$
\mathrm{E}_{\mathrm{c}}=3.17 \mathrm{U}_{\mathrm{p}}+\mathrm{I}_{\mathrm{p}}
$$

Previous experimental results $[69,70]$ match well with the theoretical cutoff energy based on Eq.2.12.

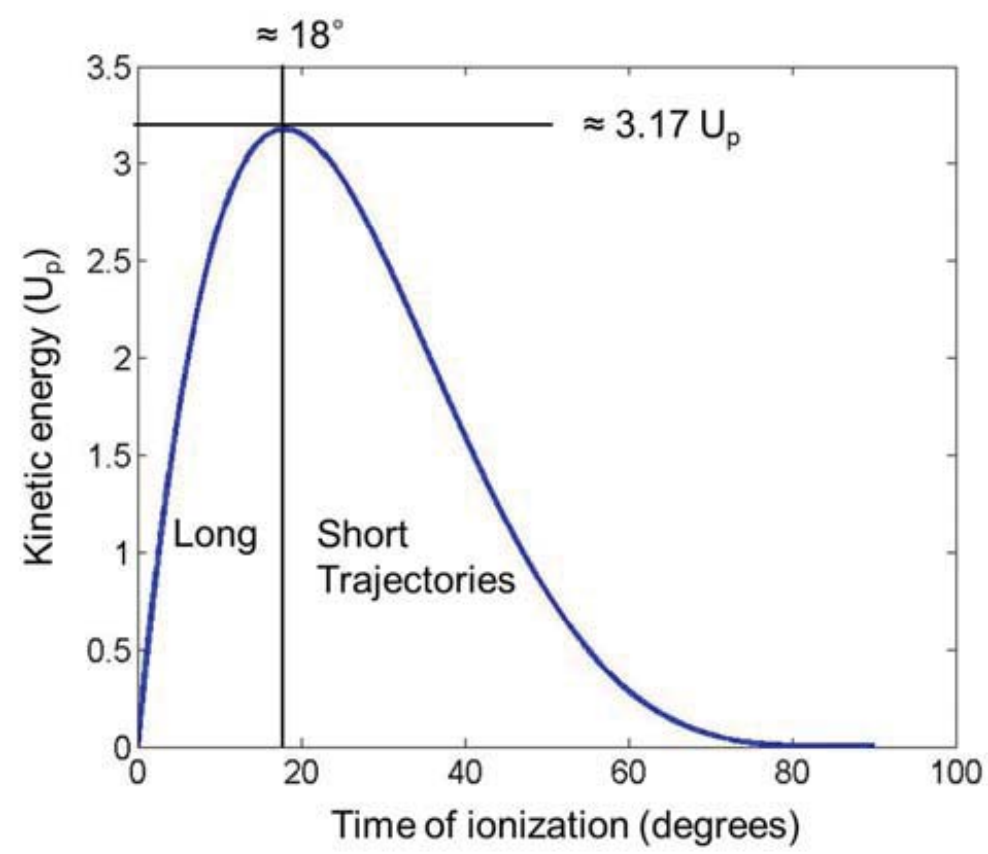

Figure 2.3: Return kinetic energy of an electron to its parent ion as a function of time of ionization, the latter expressed as phase of the drive laser at the instance of ionization.

The described process of ionization and recombination process repeats twice every optical cycle, and this doubling of the periodicity leads to doubling of the spectral spacing of the generated harmonics in the spectral domain. This is why only the odd harmonic orders appear in the HH spectrum while the even order harmonics undergo destructive interference. The three-step model implies that only linear 
polarized laser field is suitable for HHG because any slight ellipticity will direct the electron trajectory away from the parent ion, preventing the recombination of the electron with its parent ion. Previous experiment [71] has shown a decrease in harmonic field with elliptical polarized laser field.

From the above discussion, two of the electron trajectories contribute to majority of the harmonic emission. One is called the short trajectory where the return time is short compared to the cutoff timing, whereas the other has a longer return time, close to one optical period. The phase accumulated by the electron upon recombination with the ion, as it freely propagates following one of these trajectories, is called intrinsic phase and is given as

$$
\phi_{i}(\mathrm{r}, \mathrm{t})=\mathrm{a}_{i} \mathrm{I}(\mathrm{r}, \mathrm{t}) .
$$

Here, $a_{i}$ is the slope of the phase as a function of intensity for the corresponding electron trajectory, $i=$ long, short and $\mathrm{I}(\mathrm{r}, \mathrm{t})$ is the spatio-temporal dependent intensity of the drive laser, where $t$ is time and $r$ is the radial coordinate. The value for $\alpha_{\text {long }}$ is found to be much larger than that of $\alpha_{\text {short }}$ [72]. From Eq.2.13, we can see that the intensity variation can influence both the spatial and spectral characteristics of the harmonic emission. In spatial domain, the intensity variation leads to a curvature of the phase front, which make a strongly divergent harmonic emission for the long trajectory [73] and collimated harmonic emission for the short trajectory, since a short is approximately independent of the intensity [74]. In spectral domain, the intensity variation can result in a change in the instantaneous frequency, which gives rise to frequency shift, chirp and bandwidth broadening of the harmonic [75]. A more detailed analysis of the spectral control of the highharmonic will be discussed in chapter 5 .

Overall, the microscopic picture of HHG is helpful to understand some features of the HHG spectrum, such as the maximum achievable photon energy or the plateau-shape of the spectrum with odd-only harmonics. However, it does not provide any prediction on what an extended medium would emit; a macroscopic medium in which the emission from a large number of atoms is superimposed. This so-called macroscopic picture of HHG will be presented in the next section.

\subsection{HHG macroscopic picture}

For the high-harmonics from the single atoms to add up coherently upon propagation, the generated high-harmonics at different positions in the medium have to interfere constructively. This happens when the drive laser (and thereby also the induced wave of nonlinear polarization) and the emitted high-harmonic waves have the same phase velocity, which is a condition known as phase-matching. However, due to dispersion, which is a general property of all media, there will be a phase- 
mismatch between the drive laser and the high-harmonic waves. The mismatch in phase velocity, $v_{p h}$, between the two can be expressed as a mismatch in wave vectors, $\Delta k=\omega / \Delta v_{p h},[54]$

$$
\Delta \mathrm{k}=\mathrm{qk}_{\mathrm{IR}}-\mathrm{k}_{\mathrm{XUV}}=\Delta \mathrm{k}_{\mathrm{at}}-\Delta \mathrm{k}_{\mathrm{el}}-\Delta \mathrm{k}_{\mathrm{g}},
$$

and where $k_{I R}$ and $k_{X U V}$ are the wave vectors of drive laser and high-harmonic waves and where $q$ is the high-harmonic order. From Eq. 2.13, it can be seen that the wave vector mismatch, contains three contributions. The first contribution, $\Delta k_{a t}$, results from the atomic dispersion, i.e., from the difference in refractive index at the drive laser frequency, $\omega$, and the harmonic frequency, $q . \omega$, which is given as

$$
\Delta \mathrm{k}_{\mathrm{at}}=\frac{2 \pi \mathrm{q}}{\lambda}(1-\eta) \mathrm{P} \Delta \mathrm{n} .
$$

Here $\eta$ is the fraction of free electrons with respect to the neutral atoms, $q$ is the harmonic order, $P$ is the gas pressure in atmospheres, and $\Delta n$ is the difference between the refractive indices of the neutral gas for the drive laser $(n(\lambda))$ and harmonic $(n(\lambda / q))$ at atmospheric pressure. The second contribution, $\Delta k_{e l}$ comes from the dispersion due to the presence of free electrons which is given as

$$
\Delta \mathrm{k}_{\mathrm{el}}=-\mathrm{P \eta N} \mathrm{N}_{\mathrm{atm}} \mathrm{r}_{\mathrm{e}} \lambda\left[\frac{\mathrm{q}^{2}-1}{\mathrm{q}}\right],
$$

where $r_{e}$ is the electron radius and $\mathrm{N}_{\mathrm{atm}}$ is the atomic number density at atmospheric pressure. From Eq.2.14 and 2.15, it can be seen that both scale with pressure but here an opposite sign. The third contribution, $\Delta k_{g}$ comes from the geometry dispersion that is introduced via the geometry of the wave propagation, i.e. whether the drive is e.g. focused or waveguided, which is independent of the pressure. For instance, for HHG in a gas jet or gas cell, and using a Gaussian drive laser beam that is focused, the Gouy phase shift that occurs in a focus has to be taken into account and is given as

$$
\Delta \mathrm{k}_{\mathrm{g}}=(\mathrm{q}-1) \frac{2}{\mathrm{~b}}
$$

where $b$ is the confocal parameter which is defined as twice the Rayleigh length. For HHG in a gas-filled capillary, where the capillary acts as a waveguide for the drive laser, $\Delta k_{g}$ is given by the waveguide dispersion which can be expressed as 


$$
\Delta \mathrm{k}_{\mathrm{g}}=-\frac{\mu_{l m}^{2} \mathrm{c}}{2 \mathrm{a}^{2} \omega}\left[\frac{\mathrm{q}^{2}-1}{\mathrm{q}}\right]
$$

where $\mu_{l m}$ is $\mathrm{m}^{\text {th }}$ root of the $l^{\text {th }}$-order Bessel function while $a$ is the capillary radius. Eq. 2.13 shows that phase-matching $(\Delta k=0)$ is achieved when the contribution from the geometry and the free electrons are balanced by the atomic dispersion term. This can be achieved by choosing an according pressure which we call the phasematching pressure. From the phase matching condition, we also see that if we increase the drive intensity further, at some point we will reach a critical ionization, $\eta_{c}$, at which the phase-mismatch introduced by the free electrons can no longer be balanced by another contribution [54]. The intensity at which the critical ionization is reached is called the saturation intensity which limits the high-harmonic cutoff.

\subsection{Drive laser propagation}

When an ultrashort laser pulse propagates through a medium, high-harmonic might be generated. But, in addition, there are several processes that modify the spatial, temporal or spectra of the pulse, which can have a strong influence on the highharmonic generation process. For the experiments presented in this thesis, we have to consider the influence of waveguiding in a capillary, of various cross sections and lengths, whereas the medium in the capillary is a noble gas, and where plasma is generated by ionization of the gas. The waveguided drive laser can maintain relatively high intensity over a long interaction length; extending to several times the Rayleigh length and counteracting the ionization-induce defocusing. The next subsections describe several possible propagation effects which can influence the drive laser pulse during HHG.

\subsubsection{Waveguide propagation}

The spatial distribution of a high-quality laser beam in free space, i.e., where can often be described by the Hermite-Gaussian modes (so-called Gaussian beams); where the electric field does not have to fulfil any boundary conditions. When a laser beam travel through a capillary, the wall of the capillary sets boundary conditions such that, the electric field decays and reduces to zero beyond the wall, while any light reflected by the wall keeps travelling through the capillary.

The repeatedly reflected field satisfying this boundary condition are known as Bessel modes and were first reported by Marcatili et. al [76]. In an experiment, to make use of the waveguiding in HHG, the drive laser Gaussian beam are coupled into Bessel modes that are provided by the capillary. We consider here a linearly polarized drive laser, and therefore the pulse will only excite linearly polarized 
modes within the capillary. The modes are called hybrid $E H_{l m}$ modes and the electric field profile for these modes versus radius, $r$, is given by

$$
\mathrm{E}_{\mathrm{y}, 1 \mathrm{~m}}=\mathrm{J}_{0}\left(\mathrm{u}_{1 m} \frac{\mathrm{r}}{\mathrm{a}}\right) \text {. }
$$

Where $J_{0}$ is the $0^{\text {th }}$ order Bessel function, $\mathrm{u}_{1 \mathrm{~m}}$ is the $\mathrm{m}^{\text {th }}$ root of $J_{0}$, and $a$ is the inner radius of the capillary. The propagation of these modes can be expressed in terms of a complex wave vector as follow [76]:

$$
\gamma_{1 \mathrm{~m}}=\beta_{1 \mathrm{~m}}+\mathrm{i} \alpha_{1 \mathrm{~m}} .
$$

In this expression, $\beta_{1 \mathrm{~m}}$ is the propagation constant and $\alpha_{1 m}$ is the attenuation coefficient and are given as

$$
\begin{aligned}
& \beta_{1 \mathrm{~m}}=\mathrm{k}_{0}\left[1-\frac{1}{2}\left(\frac{\mathrm{u}_{1 \mathrm{~m}} \lambda}{2 \pi \mathrm{a}}\right)^{2}\right] \\
& \alpha_{1 \mathrm{~m}}=\left(\frac{\mathrm{u}_{1 \mathrm{~m}}}{2 \pi}\right)^{2}\left(\frac{\lambda^{2}}{\mathrm{a}^{3}}\right)\left(\frac{\frac{1}{2}\left(\mathrm{n}^{2}+1\right)}{\sqrt{\mathrm{n}^{2}-1}}\right),
\end{aligned}
$$

where $k_{0}$ is the propagation constant in vacuum and $n$ is the refractive index of the dielectric material of the capillary waveguide walls.

$$
A_{1 m}(z)=A_{1 m}(0) e^{\alpha_{1 m} z} .
$$

As the attenuation coefficient, $\alpha_{\mathrm{nm}}$, is proportional to the square of $u_{1 \mathrm{~m}}$, higher-order modes $(m>1)$ are more lossy than lower order modes where the amplitude of the modes as a function of distance, $z$ is given by Eq.2.2. The energetic coupling efficiency from a freely propagating, Gaussian drive laser beam into a guided Bessel mode can be determined using the overlap integral of a Gaussian with a Bessel function, that the Gaussian beam waist is placed at the entrance of the capillary [77]:

$$
\eta_{\mathrm{m}}=\frac{\left[\int_{0}^{\mathrm{a}} \exp \left(-\frac{\mathrm{r}^{2}}{\mathrm{w}^{2}}\right) \mathrm{J}_{0}\left(\mathrm{u}_{1 \mathrm{~m}} \frac{\mathrm{r}}{\mathrm{a}}\right) \mathrm{rdr}\right]^{2}}{\int_{0}^{\infty} \exp \left(-\frac{2 \mathrm{r}^{2}}{\mathrm{w}^{2}}\right) \mathrm{rdr} \int_{0}^{\mathrm{a}} \mathrm{J}_{0}^{2}\left(\mathrm{u}_{1 \mathrm{~m}} \frac{\mathrm{r}}{\mathrm{a}}\right) \mathrm{rdr}},
$$

where $w$ is the Gaussian beam radius at the beam waist (waist radius). The coupling efficiency for the first five $E H_{l m}$ modes is shown in Fig. 2.4 as a function of the ratio of waist radius to capillary radius $(w / a)$. From the plot, it shows that the lowest order mode $E H_{11}$ possesses a maximum coupling efficiency of $\eta_{1}=98.1 \%$, if the ratio is chosen as $w / a=0.64$. Therefore, it is important in choosing the correct 
waist size of the drive laser for optimal coupling into the capillary. However, we note that a real laser beam can often be non-Gaussian $\left(\mathrm{M}^{2}>1\right)$ or elliptical where the actual coupling efficiency to the $E H_{11}$ mode, $\eta_{1}$ become less than the theoretically predicted value of $98.1 \%$, even when we choose the ideal ratio for $w / a$.

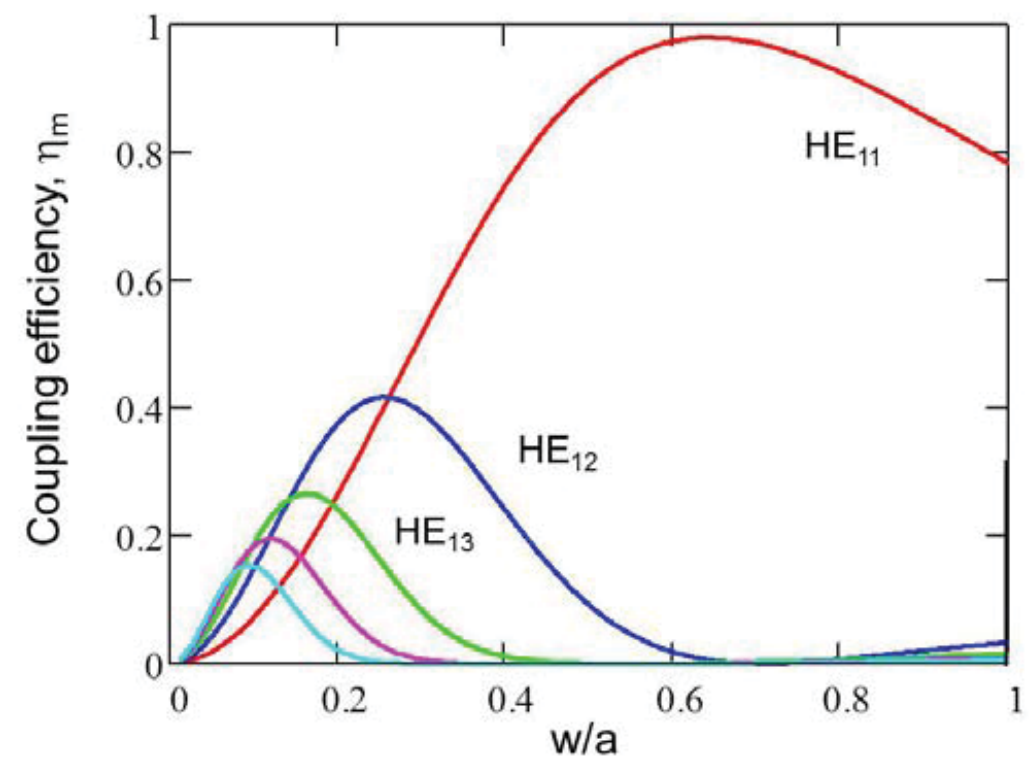

Figure 2.4: The coupling efficiency $\eta_{\mathrm{m}}$ for the first five $E H_{l m}$ modes as a function of the ratio of drive laser beam waist radius to capillary radius $(w / a)$.

\subsubsection{Nonlinear Kerr effects}

The optical Kerr effect is a linear change in refractive index of a material in response to the applied light intensity. This causes the refractive index of any material to become intensity-dependent, in an almost instantaneous fashion which can be approximated as

$$
\mathrm{n}(\mathrm{I}(\mathrm{r}, \mathrm{t}))=\mathrm{n}_{0}+\mathrm{n}_{2} \mathrm{I}(\mathrm{r}, \mathrm{t}) \quad\left(\mathrm{n}_{2}>0\right) .
$$

Where $n_{0}$ is the linear refractive index and $n_{2}$ is a small expansion coefficient called Kerr index which is proportional to the third-order nonlinear susceptibility. In most material, also in the gas used here for HHG, the Kerr index, $n_{2}$ is greater than zero and therefore the total refractive index will increase with increasing intensity. Since the drive intensity varies transversely in space and also temporally, refractive index varies spatially and temporally as well. The temporal variation of the refractive index gives rise to self-phase modulation while spatial variation leads to selffocusing. For quantitative consideration it is useful to introduce the self-phase modulation length, $\mathrm{L}_{\text {gas }}$, which is the length over which the maximum on-axis 
nonlinear phase shift, $\Delta \Phi=n_{2} \omega_{0} I_{0} L / c$, has become equal to unity. $\mathrm{L}_{\text {gas }}$ SPM is thus given as

$$
\mathrm{L}_{\mathrm{gas}}=\frac{\mathrm{c}}{\omega \mathrm{n}_{2} \mathrm{I}}
$$

For a Gaussian beam one obtains the self-focusing length, $\mathrm{L}_{\text {gas }}$ [5]

$$
\mathrm{L}_{\text {gas }_{\mathrm{SF}}}=\frac{2 \mathrm{n}_{0}}{0.61} \frac{\mathrm{w}_{0}^{2}}{\lambda} \frac{1}{\left(\mathrm{P} / \mathrm{P}_{\mathrm{cr}}\right)^{1 / 2}}
$$

where $w_{0}$ is the beam waist radius, $P$ is the drive laser power, and $P_{c r}=\lambda_{0}{ }^{2} / 8 \mathrm{n}_{0} \mathrm{n}_{2}$, with $\lambda_{0}$ the vacuum wavelength. Another nonlinear propagation effect that is caused by the intensity dependent index is self-steepening of the pulse. The self-steepening process reduces the group velocity with which the peak of the pulse propagates and thus leads to a steepening of the trailing part of the pulse. A quantitative expression can be obtained via the nonlinear group index defined as $n_{2}{ }^{(g)}=n_{2}+\omega\left(d n_{2} / d \omega\right)$ which yields an effective length over which self-steepening is significant is given by

$$
\mathrm{L}_{\text {gas }_{S S}}=\frac{c \mathrm{~T}}{\mathrm{n}_{2}^{(\mathrm{g})} \mathrm{I}}
$$

where $\mathrm{T}$ is the full width pulse duration measured at $1 / e$ intensity.

\subsubsection{Nonlinear plasma effects}

Next, the spatially and temporally varying index due to ionization during the pulse also affects the refractive index. The ionization increases the density of electrons (plasma) which corresponds to a lowering plasma index:

$$
n_{p}(r, t)=\sqrt{1-\frac{\omega_{p}^{2}(r, t)}{\omega^{2}}}
$$

where the plasma frequency, $\omega_{p}$, is defined as

$$
\omega_{p}(r, t)=\sqrt{\frac{N_{e}(r, t) e^{2}}{\varepsilon_{0} m_{e}}},
$$

where $N_{e}(r, t)$ is the electron density. Again, because the electron is a (fast rising) function of time the resulting temporal variation of refractive index gives rise to 
self-phase modulation of the drive laser. The typical length over which the plasma index will significantly change the spectrum via ionization-induced self-phase modulation can be calculated as

$$
\mathrm{L}_{\text {Plasma_SPM }}=\frac{\mathrm{c}}{\omega \mathrm{n}_{\mathrm{p}}} .
$$

Due to self-phase modulation, another effect called the ionization induced blue shift can be observed. From Eq.2.28 it can be seen that an increasing electron density gives rise to a lowering of $n_{p}$. Therefore, a decreasing $n_{p}$ will shift the spectrum towards the blue. A red shift in the spectrum due to a rising $n_{p}$ from a decrease of the free electron will usually not be observed in experiment with femtosecond-pulses because plasma recombination happens at a longer timescales $(\sim \mathrm{ns})$ in which the ultrashort ( fs) drive pulse has passed. The frequency shift of the drive laser in weakly ionized gases can thus be expressed as

$$
\Delta \omega=\frac{-\omega_{0}}{c} \int_{0}^{L} \frac{\delta n_{p}}{d t} d z .
$$

Where $\omega_{0}$ is the drive laser frequency, $z$ is the axis where the drive laser propagates along and $L$ is the interaction length in the gas medium. Lastly, we consider the spatial variation of the refractive index provided by the spatial distribution of the electron density. On axis of the laser beam, the electron density will be higher; accordingly (Eq.2.28) there will be a lowering of the index on axis. When the electron density variation is strong enough, this gives rise to defocusing, which can be expressed via certain defocusing length for the propagating pulse. The defocusing length, $\mathrm{L}_{\mathrm{def}}$, where its divergence doubles can be calculated as

$$
\mathrm{L}_{\mathrm{def}}=\frac{\lambda}{2} \frac{\varepsilon_{0} \mathrm{~m}_{\mathrm{e}} \omega^{2}}{\mathrm{~N}_{\mathrm{e}} \mathrm{e}^{2}} .
$$

Defocusing is usually an undesired effect in nonlinear optical conversion, because it lower the drive laser intensity and thereby the conversion efficiency. However, when using a capillary, waveguiding of the beam keep the beam focused and the drive intensity can be maintained over a longer interaction length. Lastly also, the plasma reduced index variation might induce a self-steepening, and its effective length is given by

$$
\mathrm{L}_{\text {Plasma_Ss }}=\frac{\mathrm{cT}}{\mathrm{n}_{\mathrm{p}}} \quad \text {. }
$$


From the above equations, it can be seen that the effective length of the nonlinear Kerr and plasma effects becomes shorter for higher plasma density, which can occur when the drive intensity is increased, or when the gas density (gas pressure) is increased. As long as the interaction length in the gas medium is shorter than the effective length for the described processes, the drive laser pulse will not be significantly reshaped temporally or spatially via nonlinear propagation effects, such that its propagation can be described solely via linear effects, such as dispersion, absorption, focusing and waveguiding.

\subsection{Conclusions}

In this chapter, we have looked at some general aspect of high-harmonic generation (HHG) which is the underlying process for the coherent soft-X-ray source presented in this thesis. The harmonic radiation comes not only from a single atom response to the driving electric field, but also from the sum of all the harmonics radiated as the drive laser propagate through an ensemble of atoms which is dependent on phasematching. We have also looked into the possible mechanisms which can lead to a change in drive laser propagation within a capillary waveguide. This is important because drive laser propagation can influence the single atom response as well as the phase-matching in the HHG process. 


\section{3}

\section{Single-shot fluctuations in waveguided high-harmonic generation}

\subsection{Introduction}

High-harmonic generation (HHG) is a nonlinear optical process that provides coherent radiation in the form of ultra-short pulses, covering a broad spectrum including the extreme ultraviolet (XUV). The process is typically driven by femtosecond pulses focused to high intensities (in the order of $10^{14} \mathrm{~W} / \mathrm{cm}^{2}[45,78]$ ) in samples of noble gas, which are usually supplied in the form of gas-jets $[35,45]$, gas-cells [53, 79, 80] or in thin, gas-filled capillaries [54, 81]. Conversion efficiencies reach values of up to $10^{-5}$ to $10^{-6}$, for instance using Argon [57, 82-86].

A fundamental property of HHG associated with the inherent high nonlinearity of the process is that all parameters of the output radiation fluctuate from pulse to pulse, and that the spatial coherence becomes reduced via distortions of the output beam cross section and phase fronts. For applications of HHG it is of central importance to characterize these fluctuations and distortions, such as for increasing the measurement precision for absolute, nonlinear ionization cross sections [58, 59]. Other examples requiring a characterization of fluctuations and distortions include lens-less diffractive imaging with maximum resolution and optimum utilization of the dynamical range [44], or injection seeding at free-electron laser facilities for improving the coherence and the shot-to-shot stability of the laser output [60-62].

In geometries based on free propagation of the drive laser beam, i.e., in gas cells and gas jets, fluctuations in HHG have been characterized extensively, via recording the directional fluctuations [50, 87], fluctuations of the pulse energy [87, 88], and also spectral fluctuations [89]. In contrast to free propagation, one might expect a lower degree of output fluctuations and a higher degree of spatial coherence, when controlling the drive laser propagation through the gas sample via waveguiding. Such control is achieved when providing the gas sample inside a thin capillary, also called hollow core fiber. Here the propagation of the input pulse produced by the drive laser is confined in the form of waveguide modes [90]. Indeed, there are experimental signatures that $\mathrm{HHG}$ in capillary waveguides can be spectrally controlled, in the form of dedicated suppression or enhancement of high-harmonic orders [81, 91, 92]. Similarly, expecting reduced beam pointing fluctuations by 
waveguiding of the input pulse, it has been suggested to use capillary based HHG for injection seeding instead of free propagation of the input pulse [93].

On the other hand, one may also argue that waveguiding enhances nonlinear effects that modify the input pulse propagation to an undesired degree and, correspondingly, also enhances fluctuations in the $\mathrm{HH}$ output. For instance, via ionization-induced spectral broadening and plasma-induced refraction the drive pulses can undergo self-compression [57, 94]. Similarly, intensity dependent index changes can distort the wave fronts of the driving laser pulse, thereby exciting propagation in multiple waveguide modes (higher-order modes), the superposition of which creates spatio-temporal hot spots that further distort the drive laser propagation [57, 95]. Eventually, there might also be simple, direct effects. For instance, pointing fluctuations might be turned into intensity fluctuations of the driving laser pulse inside the capillary because a capillary waveguide acts as a spatial mode filter for the incident beam. The signature of such effects should be a correlation of the fluctuations in the $\mathrm{HH}$ output with drive laser beam pointing fluctuations.

Surprisingly, to our knowledge, there is no characterization or quantification of shot-to-shot (single-pulse) fluctuations or shot-to-shot output beam deformations in waveguided HHG. Changes of the beam direction and shape have only been addressed by averaging over a large number of pulses [63]. What is rather required is to record the output parameters of single output pulses, for instance the XUV pulse energy, the divergence, or the direction of emission. Then, using series of such single-pulse recordings, one can provide a statistical analysis of pulse-to pulse (shotto shot) fluctuations, for instance in the form of standard deviations of single parameters, or in the form of a correlation of fluctuations in pairs of output parameters. To enable a comparison with HHG from jets and cells, it appears important to provide a first, basic characterization of the type and size of fluctuations that are present in capillary based HHG.

We begin in Section 3.2 with the description of a capillary based HHG setup. Then we describe the calibrations steps required to determine an absolute value for the total pulse energy in the high-harmonic beam in Section 3.3. Following that, we present the first shot-to-shot characterization of high-harmonic generation in a waveguiding geometry using Argon, where we first characterize the fluctuations of the HH pulse energy (energy jitter) in Section 3.4 followed by the directional fluctuations (beam pointing stability) and fluctuations in beam divergence of the high-harmonic $(\mathrm{HH})$ output in Section 3.5. To enable a better tracing of possible reasons for fluctuations, we characterize the correlations between various input and output parameters in Section 3.6. Finally, we conclude in Section 3.7 the major experimental results and findings. 


\subsection{Experimental setup}

The experimental setup used for HHG is schematically shown in Fig. 3.1. To generate the drive laser pulses we employ a Ti:Sapphire infrared (IR) laser system (Legend Elite Duo HP USP, Coherent Inc.) with a repetition rate of $1 \mathrm{kHz}$ and a center wavelength of $795 \mathrm{~nm}$. The laser provided almost Fourier-limited pulses with $40 \mathrm{fs}$ duration and a time bandwidth product (TBP) of 0.5 as measured with a homebuilt Grenouille [96]. The nominal maximum pulse energy of the laser is $8 \mathrm{~mJ}$ but, in order to avoid any major self-phase modulation along the path into the gas-filled capillary, we keep the pulse energy below or equal to $1.1 \mathrm{~mJ}$. A rotatable half-wave plate followed by a polarizing beam splitter is used as variable attenuator. For the maximum pulse energy we calculate [97] a maximum increase of the TBP by a factor of 1.3 at the entrance to the gas capillary, in which we included the beam path of nearly three meters through air, the half-wave plate, the focusing lens and the entrance window for transmitting the beam into the vacuum vessel that contains the capillary.

The beam is focused with a lens of $75 \mathrm{~cm}$ focal length into a $67 \mathrm{~mm}$ long capillary having an inner radius of $a=75 \mu \mathrm{m}$. This focal length is chosen to match the beam waist radius to the radius of the lowest-order waveguide mode of the capillary [77]. The capillary is mounted in the vacuum vessel at a distance of $50 \mathrm{~cm}$ from the entrance window, with the entrance of the capillary in the focal plane of the lens. For filling the capillary with gas in a controlled manner, the capillary is equipped with six $0.4 \mathrm{~mm}$ wide slits as shown in the inset of Fig. 3.1. Two of the slits, located at 5 and $42 \mathrm{~mm}$ from the entrance of the capillary, respectively, are used as gas inlets with a continuous flow and define an interaction length, $L_{m}=37$ $\mathrm{mm}$ with a constant pressure profile. The remaining four slits are evenly distributed between $45 \mathrm{~mm}$ ( $3 \mathrm{~mm}$ downstream the second slit for gas inlet) and $53.4 \mathrm{~mm}$ from the entrance of the capillary. These four slits are used for differential pumping. The capillary ends $13.6 \mathrm{~mm}$ behind the last pumping slit. The pressure is measured near the capillary inlet and should correspond to the pressure inside the capillary due to the negligible pressure drop between the point of measurement and the inside of the capillary. The interaction length in this capillary configuration provides wave guiding for the drive laser pulses over about four Rayleigh lengths of the focused beam $\left(z_{R}=9 \mathrm{~mm}\right)$. For alignment, each end of the capillary is mounted on a twoaxis translation stage. The alignment comprises maximizing the drive laser throughput and simultaneously restricting the drive laser output to lowest-order mode. We measure a maximum capillary throughput of $50 \%$. This is less than the theoretical limit of $96 \%$ but is comparable with previously reported values [56, 90]. We attribute the deviation from maximum theoretical throughput to several experimental imperfections, including a slightly elliptical beam profile (1:2 aspect ratio), a slightly off-unity beam parameter $\left(M_{x}^{2}=1.3 ; M_{y}^{2}=1.1\right)$, and that part of 
the guided light is scattered at the various slits in the capillary. Another indicator for the alignment was a visual inspection of fluorescence emitted transversely from the gas in the capillary. This gives a qualitative impression on how homogeneously the drive laser intensity is distributed along the capillary. At lower gas pressures, a homogeneous distribution of fluorescence coincided with a maximum transmission of the drive laser through the capillary.

With the minimum drive laser pulse energy of $0.6 \mathrm{~mJ}$ used and an estimate of 30 $\%$ losses due to incoupling and scattered light at the first slit, a peak intensity of up to $1.8 \times 10^{14} \mathrm{~W} / \mathrm{cm}^{2}$ can be launched into the interaction length in the waveguide. Behind the capillary, for reducing the drive laser intensity by diffraction, we let the $\mathrm{HH}$ and drive laser beams co-propagate over a distance of $1.5 \mathrm{~m}$. The drive laser beam is then blocked by a set of two $200 \mathrm{~nm}$ thick Aluminum (Al) filters placed in series. The filters act as a band pass for XUV radiation, transmitting approximately half of the $\mathrm{HH}$ radiation in the wavelength range of $17 \mathrm{~nm}$ to $80 \mathrm{~nm}$. The transmitted $\mathrm{HH}$ beam is detected with an XUV CCD camera (Andor, DO420-BN) placed $20 \mathrm{~cm}$ behind the filters. This camera position is in the far-field of the $\mathrm{HH}$ beam, as can be seen from the small Fresnel number, $N_{f}=a^{2} / L \lambda$ between 0.04 and 0.2 , calculated for the transmitted wavelength range, where $a=75 \mu \mathrm{m}$ is the radius of the capillary and $L=1.7 \mathrm{~m}$ is the distance from the capillary exit to the XUV camera. Due to the low Fresnel number, the divergence of the HH beam and the direction of emission can be straightforwardly obtained from beam profile measurements. Fig. 3.2(a) shows a typical single-shot measurement of the beam intensity profile. Comparison with a fit curve shows this profile to be near-Gaussian in both transverse directions.

For characterizing the size of shot-to-shot fluctuations in the high-harmonic output, we record series of 100 single shots. The sample size is set on one hand to obtain an error of $10 \%$ or less at $95 \%$ confidence interval for the statistically determined parameters and on the order hand measure subsequent series of shots under equal conditions. These single-shot measurements are performed at a rate of 3 $\mathrm{Hz}$, by blocking about 332 drive laser pulses per third of a second with a triggered combination of an optical chopper and a magnetic shutter. For measurements of the $\mathrm{HH}$ spectrum, a high-line-density, home-made transmission grating $(10,000$ lines $/ \mathrm{mm}$ ) [98] is moved into the $\mathrm{HH}$ beam path at $1.7 \mathrm{~cm}$ distance in front of the XUV camera. Measurements of average high-harmonic spectra are obtained by letting the camera integrate over 1000 subsequent shots. Fig. 3.2(b) shows a typical $\mathrm{HH}$ spectrum with four harmonic orders ranging from the $17^{\text {th }}$ up to the $23^{\text {rd }}$ order, recorded with an input pulse energy of $0.6 \mathrm{~mJ}$ and a gas pressure of 53 mbar. The spectrum is limited on the short-wavelength side to about $34 \mathrm{~nm}\left(23^{\text {rd }}\right.$ order). This wavelength agrees well with the calculated cut-off wavelength assuming that $50 \%$ of the drive laser energy has reached the end of the capillary which coincides with our measurement of $50 \%$ throughput efficiency for the drive laser. On the long- 
wavelength side the spectrum is limited to about $47 \mathrm{~nm}\left(17^{\text {th }}\right.$ order $)$ by strong reabsorption of the generated XUV radiation in Ar [54].

In order to devise an appropriate measurement setup for fluctuations of the $\mathrm{HH}$ pulse energy, we considered that such fluctuations may be caused via two different mechanisms. The first is an energy jitter and beam pointing fluctuation of the drive laser [87], which can be called an external effect, letting the amount of pulse energy that is coupled into the waveguide fluctuate. The second mechanism may be called intrinsic, i.e., when fluctuations are rather based on highly nonlinear effects in the capillary such as associated with spatio-temporal reshaping of the drive laser pulse inside the capillary. It has recently been observed in an identical waveguiding capillary that such reshaping is related to beating of drive laser light propagating simultaneously in the fundamental and higher-order modes that are excited through ionization-induced scattering of the driving laser pulse [57, 95]. Furthermore, the modeling in [95] showed that nonlinear optical effects like self-phase modulation or self-steepening do not significantly modify the pulse propagation in such a capillary. This is confirmed by calculating the characteristic propagation length required for these processes to become dominant [94]. We find that these are $0.1 \mathrm{~m}$ and $8.5 \mathrm{~m}$ for self-phase modulation and self-steepening, respectively. These are much longer than the capillary length of $3.7 \mathrm{~cm}$. Measurements on the spectrum of the drive laser pulse behind the capillary show no spectral broadening. This substantiates the conclusion from the modeling mentioned above and therefore we ignore the influence of these nonlinear optical processes on the drive laser pulse propagation in the remainder of this paper.

To possibly discriminate between the two mechanisms that may cause fluctuations in the HH pulse, we capture with a CMOS camera single shots of the drive laser beam profile while, at the same time, the XUV camera captures single shots of the HH beam profile. The CMOS camera is positioned in the residually transmitted drive beam behind the last folding mirror $\left(\mathrm{M}_{\mathrm{f}}\right.$ in Fig. 3.1) and is triggered for synchronous recording with the XUV camera. Based on single-shot pairs of XUV and CMOS camera images it becomes possible to search for correlations between the drive laser and HH beam energy and pointing fluctuations. 


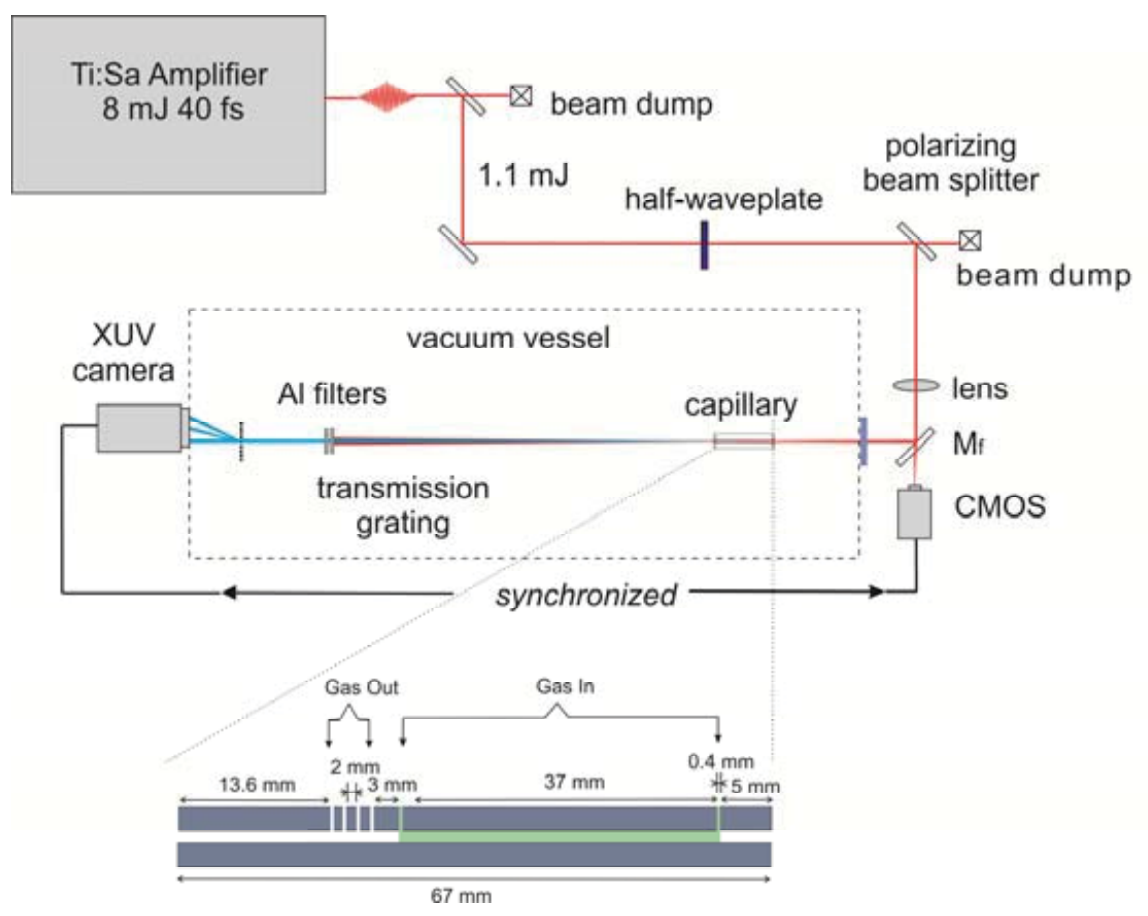

Figure 3.1: Experimental setup for high-harmonic generation (HHG) with a waveguiding, gas-filled capillary.
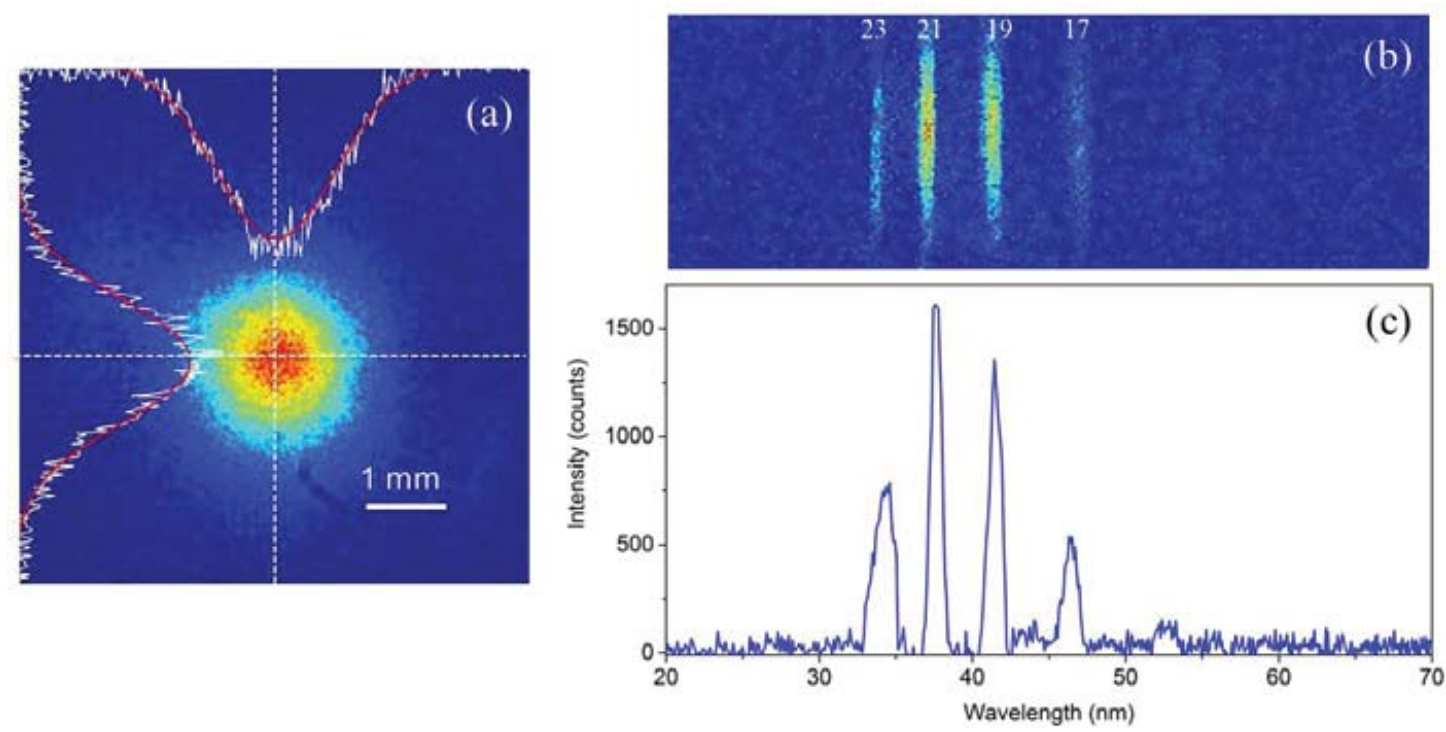

Figure 3.2: (a) Single-shot XUV camera image of the far-field high-harmonic beam cross section showing a near-Gaussian profile: the measured beam profiles (white traces) taken along the respective axes (white dotted lines) matches well with Gaussian fit curves (red). (b) Raw CCD data of highharmonic spectrum (integrated over 1000 shots) with color representing counts, horizontal and vertical axes are pixels. (c) Processed high-harmonic spectrum of (b) where intensity is plotted versus calibrated wavelength. Both measurements are taken with a drive laser pulse energy of $0.6 \mathrm{~mJ}$ (peak intensity $=1.8 \times 10^{14} \mathrm{~W} / \mathrm{cm}^{2}$ ) and an Ar pressure of 53 mbar. 
For choosing appropriate settings of the drive pulse energy and the gas pressure in the capillary, we recall that the HH output can be maximized by adjusting the gas pressure to optimize phase-matching [54], while keeping the drive pulse energy constant. The HH output can also be increased, and its spectrum can be extended towards a shorter cut-off wavelength, by increasing the pulse energy of the driving laser pulse (while readjusting the pressure) [34]. Here, we follow a heuristic approach in which we record the high-harmonic output at four different drive pulse energies (between 0.6 and $1.1 \mathrm{~mJ}$ ) and at six different gas pressures (between 40 and 160 mbar), which are chosen to include the maximum HH output.

\subsection{Determining the harmonic pulse energy}

To provide an absolute value for the total pulse energy in the high-harmonic beam, we combine the data from the measured fluence (beam profile) and spectrum. The background-corrected fluence is summed over the transverse plane to obtain the measured counts, $S$, from the corresponding CCD signal, which is a measure for the total energy in the high-harmonic beam. In order to convert $S$ into energy, we need to know the relative contribution of each harmonic, as the conversion from count to energy is wavelength dependent. The spectrum (e.g., see Fig. 3.2b) is used to determine the relative magnitude, $f_{q}$, for each harmonic and we assume that the same relative distribution of harmonics is present in the fluence measurement. The contribution, $f_{q} S$, of each harmonic can now be converted to energy using a wavelength dependent calibration factor, $c(\lambda)$, that takes into account the transmission spectrum of the $\mathrm{Al}$ filters and the responsivity of the CCD camera. The energy in a particular harmonic, $E_{q}$, can be written as

$$
E_{q}=c\left(\lambda_{q}\right) \cdot f_{q} \cdot S,
$$

and the total energy in the high-harmonic beam is obtained by summing over all harmonics. The energy calibration factor in our detection (in $\mathrm{J} /$ count) is given by $c(\lambda)=E_{e h} \sigma /\left(\eta_{Q E}(\lambda) T(\lambda)\right)$, where $E_{e h}$ is the energy required to generate an electron-hole pair in the silicon detector chip of the CCD camera $\left(E_{e h}=5.84 \times 10^{-19} \mathrm{~J}\right.$ $\cong 3.65 \mathrm{eV}), \sigma$ is the sensitivity of the CCD camera (10 electrons/count), $\eta_{Q E}(\lambda)$ is the specified quantum efficiency of the XUV camera [99], and $T(\lambda)$ is the transmission spectrum of the pair of Al filters. This spectrum is calculated from the CXRO database [100], taking into account the XUV transmission spectrum of aluminum as well as that of thin surface layers of aluminum oxide that are known to form upon contact with oxygen in air. For the calculation of the filter transmission, we assume a layer thickness of $3 \mathrm{~nm}$ (on either side) that is typical for filters stored in an oxygen free environment to minimize further oxidation $[50,82]$. We note that 
the aluminum oxide layers reduce the transmission by a factor of 2 to 2.5 across the wavelength range from 30 to $50 \mathrm{~nm}$ and therefore have to be taken into account to avoid underestimating the harmonic pulse energy.

Figure 3 summarizes the spectral variation of the quantum efficiency of the XUV camera, $\eta_{Q E}(\lambda)$, the filter transmission, $T(\lambda)$, and the calibration factor, $c(\lambda)$. It can be seen that for a wavelength of about $\lambda_{23}=34 \mathrm{~nm}$ where the strongest harmonic order is found in the measured spectra (the $23^{\text {rd }}$ ),$c\left(\lambda_{23}\right) \approx 1.2 \times 10^{-16}$ $\mathrm{J} /$ count. Alternatively expressed, an XUV pulse energy of $1 \mathrm{~nJ}$ corresponds to about $8 \times 10^{6}$ counts for the $23^{\text {rd }}$ harmonic.

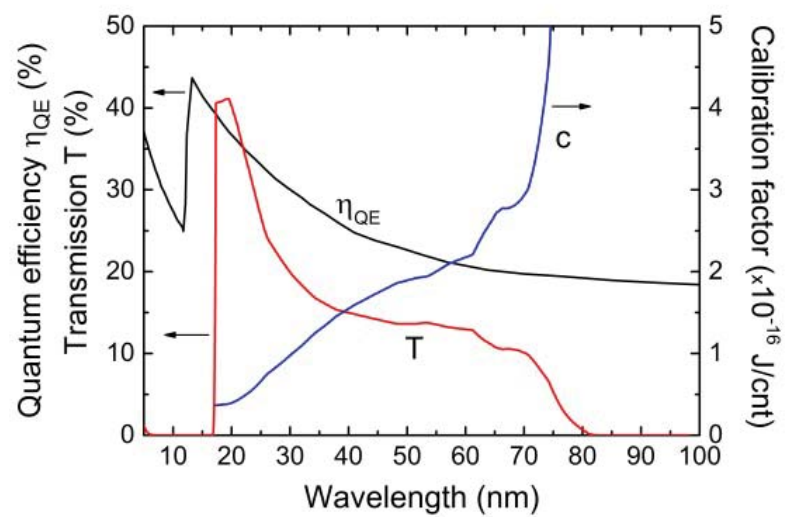

Figure 3.3: Typical quantum efficiency $\left(\eta_{Q E}\right)$ of the XUV CCD camera, the transmission $(T)$ of the filter set of two Al filters (each $200 \mathrm{~nm}$ thick) with an oxide layer on each side ( $3 \mathrm{~nm}$ thick), and the calibration factor $(c)$ versus wavelength.

\subsection{High-harmonic beam profile, pulse energy and energy jitter}

For a first qualitative overview we present in Fig. 3.4 four typical single-shot CCD images of high-harmonic beam profiles (fluence), recorded with four different drive pulse energies $(0.6,0.8,1.0$, and $1.1 \mathrm{~mJ})$ at the same pressure $(53 \mathrm{mbar})$. This particular pressure was selected because it yielded the highest HH pulse energy. For the lowest drive laser energy of $0.6 \mathrm{~mJ}$ we observe that in all shots the $\mathrm{HH}$ output exhibits a round, near Gaussian profile such as in Fig. 3.4a. At higher pulse energies $(0.8 \mathrm{~mJ}$ and most obvious at 1.0 and $1.1 \mathrm{~mJ})$ the beam profile of each shot shows shot-to-shot fluctuations and becomes increasingly distorted in a complex manner that deviates noticeably from a Gaussian profile. Especially at the two higher pulse energies, the beam profiles vary strongly from shot to shot.

We address this transition from a round and stable profile to a deformed and fluctuating profile to the onset of significant ionization of the Argon gas at drive energies above $0.6 \mathrm{~mJ}$. The spatial variation in ionization (i.e., refractive index) 
leads to scattering of the input pulse in higher-order modes, while the fast variation of the ionization with time leads to broadening of the spectrum of the input pulse. The spectral broadening and nonlinear mode mixing change the spatiotemporal profile of the driving laser pulse [57]. Note that this is strongly related to ionizationinduced defocusing dynamics that can be observed in HHG using free-space propagation of the driving laser pulse [101]. The threshold electron density $n_{t h}$ for multi-mode propagation of the driving laser pulse is given by [102]

$$
n_{t h}=\frac{m c_{0}{ }^{2}}{4 \pi e^{2}} \frac{u_{12}^{2}-u_{11}^{2}}{a^{2}} \cong \frac{7}{a^{2}} \times 10^{12} \mathrm{~cm}^{-3}
$$

where $m$ is the electron mass, $c_{0}$ is the speed of light in vacuum, $e$ is the electron charge, $u_{11}$ and $u_{22}$ are the first and second zeros of the zeroth-order Bessel function and $a$ is the radius of the capillary (expressed in $\mathrm{cm}$ in the numerically evaluated form). The ionization induced electron density can be calculated via the Ammosov-Delone-Krainov (ADK) model [67], showing that the electron density grows with the gas density (gas pressure) and the drive pulse intensity. For the parameters of Fig. 3.4, Eq. 3.2 predicts a threshold for the electron density of $n_{\text {th }}=$ $1.3 \times 10^{17} \mathrm{~cm}^{-3}$ and from the ADK model we obtain an intra-capillary pulse energy of $0.5 \mathrm{~mJ}$ to reach this threshold electron density. Due to in-coupling losses at the entrance of the capillary and scatter losses at the slits, we find that for the lowest input pulse energy (at the entrance of the capillary) of $0.6 \mathrm{~mJ}$ the electron density will remain below the threshold, at $0.8 \mathrm{~mJ}$ the electron density will be above but near the threshold, and the other two pulse energies are well above the threshold. As stated, we expect that the shape of the IR guided mode field becomes significantly affected in the latter regime [54,57], and that the phase matching conditions based on the excitation of single fundamental mode propagation [103] will be severely degraded [57]. This should cause the beam profile of the generated $\mathrm{HH}$ to deviate noticeably from a round and near-Gaussian shape. At the same time, the strong, optically nonlinear coupling of drive laser light travelling in a number of excited waveguide modes should amplify any small fluctuations of other experimental parameters that would otherwise have only negligible influence. From our experimental observations that $\mathrm{HH}$ beam deformation and fluctuations set in where multimode propagation of the driving laser pulse is expected, we conclude that the nonlinear dynamics of the driving laser pulse described in [54, 57, 103] and [94] is also the reason for our observation of shot-to-shot fluctuations. Inducing such dynamics deliberately might increase the HH output significantly [14] but it is less preferred if high-quality XUV beams with low fluctuations are required. 

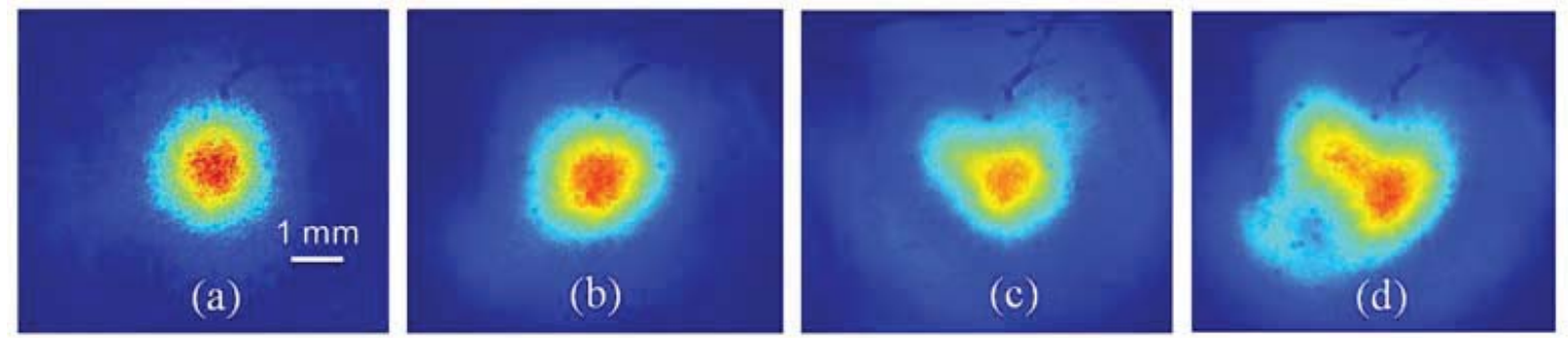

Figure 3.4: Typical single-shot images of the high-harmonic beam profile (fluence) $v s$. increasing drive laser pulse energy at a gas pressure of 53 mbar: (a) $0.6 \mathrm{~mJ}$ (b) $0.8 \mathrm{~mJ}$ (c) $1.0 \mathrm{~mJ}$ (d) $1.1 \mathrm{~mJ}$, measured with an XUV CCD at a distance of $1.7 \mathrm{~m}$ from waveguide capillary.

To identify the experimental parameters that yield a maximum $\mathrm{HH}$ output we record the output for a number of different gas pressures and drive laser pulse energies. Figure 5a shows the total (spectrally integrated) HH output pulse energy averaged over 100 single shots versus pressure for different drive laser energies. It can be seen that for all four values of the drive pulse energy, the maximum average pulse energy is obtained at around 53 mbar of pressure, which we address to optimum phase matching at this pressure. For the $0.6 \mathrm{~mJ}$ input pulse energy, where single-mode propagation of the driving laser pulse is expected, we find that the observed pressure of 53 mbar for optimum phase matching agrees well with the value predicted by the single-mode model of Constant et al. [104] for our parameters.
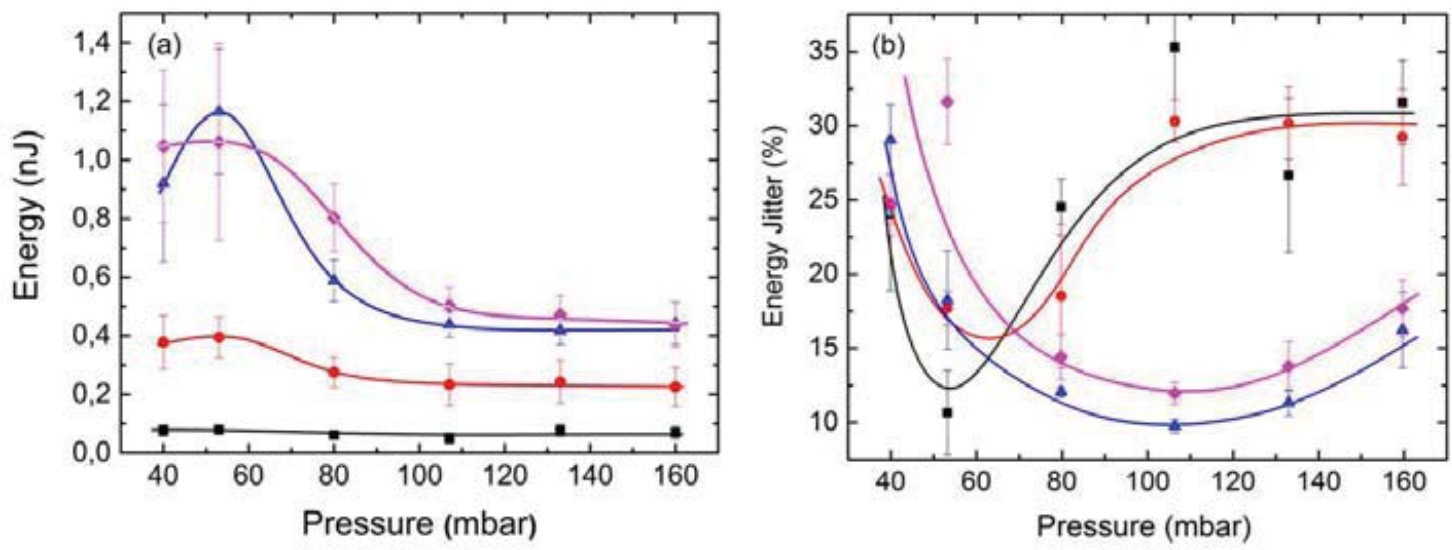

Figure 3.5: (a) Total (spectrally integrated) pulse energy of the high-harmonic beam measured at the XUV camera as a function of Argon pressure for different drive laser energies at $0.6 \mathrm{~mJ}$ (black square), $0.8 \mathrm{~mJ}$ (red circles), $1.0 \mathrm{~mJ}$ (blue triangles), and $1.1 \mathrm{~mJ}$ (purple diamonds). Each data point is obtained via measuring the single-shot pulse energy for 100 shots and subsequently calculating the average value and standard deviation (error bars). (b) Relative energy jitter, defined as the standard deviation normalized to the average pulse energy.

For quantifying the fluctuations of the output energy (energy jitter), we plot in Fig. $3.5 \mathrm{~b}$ the standard deviation of the measured pulse energy normalized to its average. This provides the relative energy jitter of high-harmonic generation as a function of gas pressure. It can be seen that, for the two lower drive laser pulse 
energies $(0.6$ and $0.8 \mathrm{~mJ})$, the jitter is lowest at $53 \mathrm{mbar}(12 \%$ and $20 \%$, respectively), where also the average output is highest. As discussed before, this is where phase matching with the fundamental waveguide mode occurs. A possible explanation for lowest fluctuations at best phase matching is as follows. At phase matching, to first order and within the phase-matching bandwidth, the $\mathrm{HH}$ output does not vary with any parameter that enters into the wave-vector balance. For instance, if there are shot-to-shot fluctuations in the degree of ionization, phase matching would remain fulfilled to first order and the $\mathrm{HH}$ output would not fluctuate as strongly. When the pressure is tuned away from the optimal value for phase matching we observe a simultaneous decrease in $\mathrm{HH}$ yield and increase in relative fluctuations. Due to the higher pressure, the electron density surpassed the threshold, even at the lowest input energy. We therefore expect multimode propagation for the driving laser pulse at these pressures and therefore a higher relative fluctuation as the HHG process becomes more susceptible to small perturbations.

The two higher input pulse energies $(1.0$ and $1.1 \mathrm{~mJ})$ show a different behavior. For these input pulse energies high fluctuations are found near the maximum HHG output at 53 mbar, while the fluctuations are strongly reduced for higher pressures. High fluctuations are expected for multi-modal propagation of the driving laser pulse, and this observation is consistent with the observed distortions in the beam profiles at a pressure of 53 mbar (see Figs. $3.4 \mathrm{~b}$ and c). For both pulse energies, the lowest fluctuations occur around a pressure of 106 mbar (see Fig. 3.5b) where the $\mathrm{HH}$ yield is lower. It can also be seen that the range of low fluctuation is rather wide, reaching almost 100-mbar width for 1-mJ input pulse. At the same time we observe that in this range the beam profile is very similar to that shown in Figs. 3.4a and $b$ and stable from shot-to-shot. This suggests that under these conditions, i.e., high pressure and high pulse energy, the complex, ionization-induced nonlinear dynamics creates a single propagating mode for the driving laser pulse [95]. Although this mode will be very different from the fundamental (empty) waveguide mode, it will produce stable and reproducible XUV output in a similar way.

Our results shows that, depending on the pressure and drive laser intensity, there is a best compromise between achieving the highest pulse energy and the lowest energy jitter. This should be taken into consideration when designing a $\mathrm{HH}$ source for specific application.

\subsection{Measurement of harmonic beam divergence and pointing stability}

To characterize the spatial beam fluctuations, we first measure the average divergence of the $\mathrm{HH}$ output versus pressure and drive laser pulse energy. We define the half-angle divergence as the ratio of the beam radius to the axial distance $(L)$ of 
the XUV CCD camera $(L=1.7 \mathrm{~m})$. The beam radius is taken as $2 \sigma_{x}\left(2 \sigma_{y}\right)$, where $\sigma_{x}\left(\sigma_{y}\right)$ is the standard deviation of the background-corrected fluence distribution in the $x(y)$-direction. The divergence is calculated for 100 shots separately and then both the average and standard deviation are calculated for each set of 100 shots. In Fig. 3.6 we plot the average and the standard deviation of the divergence in both the horizontal (Fig. 3.6(a)) and vertical (Fig. 3.6(b)) direction versus pressure for different input pulse energies. The error bars represent the standard deviation. It can be seen that the horizontal beam divergence lies in the range of 1.2 to $1.6 \mathrm{mrad}$ and shows only a weak increase with pressure and with pulse energy. The vertical beam divergence shows a stronger variation with pressure and is found to be in the range of 1.3 to $2.1 \mathrm{mrad}$ (Fig. 3.6(b)). Finally, it can also be seen that the horizontal beam divergence is generally less than the vertical divergence for the collection of input pulse energies and Ar gas pressures investigated.

A dependency on the energy of the driving laser pulse is to be expected as, in first-order approximation, the induced single-atom dipole phase is proportional to the instantaneous intensity of the driving laser pulse. The intensity of the driving pulse inside the capillary is itself a result of a highly nonlinear dynamical process $[7,12,14,29,42]$, as described above. It is therefore surprising that the observed divergence only shows a weak dependence on input pulse energy and gas pressure. However, using a modified multimode generalized nonlinear Schrödinger equation, Anderson et al. numerically showed that the size of the most intense part of the driving laser pulse and its intensity only weakly depend on beam energy and gas pressure for most of the parameter range we investigated [95], which is consistent with the weak dependence of the measured divergence on input pulse energy and gas pressure. The half-width at half of the maximum intensity of the driving laser pulse is predicted by their model to be around $10 \mu \mathrm{m}$, which agrees well with the apparent source radius $r_{a} \approx 8 \mu \mathrm{m}$ calculated from $r_{a}=\frac{\lambda}{\pi \theta}$, where $\lambda=38 \mathrm{~nm}$ is the wavelength of the strongest harmonic and $\theta \approx 1.5 \mathrm{mrad}$ is an average value for the measured far field divergence (cf., Fig. 3.6). The difference in horizontal and vertical divergence may be due to the slight elliptical shape of the input beam, leading to a slight asymmetry in the nonlinear dynamics for the driving laser pulse inside the capillary and therefore a slightly elliptical apparent source for the XUV radiation. 

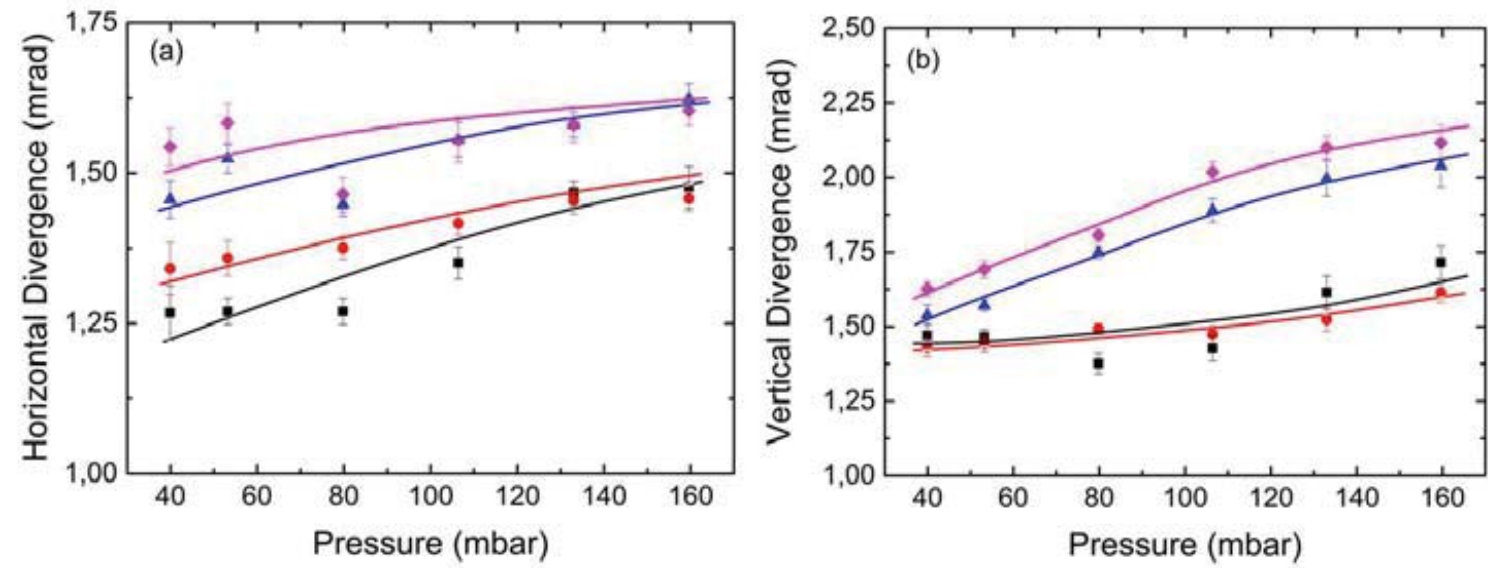

Figure 3.6: Average beam divergence of the high-harmonic output measured as a function of gas pressure for different drive laser intensities at $0.6 \mathrm{~mJ}$ (black squares), $0.8 \mathrm{~mJ}$ (red circle), $1.0 \mathrm{~mJ}$ (blue triangles) and $1.1 \mathrm{~mJ}$ (purple diamonds): (a) for the horizontal direction and (b) for the vertical direction. The error bars represent the standard deviation.

For quantifying the first-order spatial fluctuation of the HH beam, we define the relative beam pointing fluctuation in the horizontal (vertical) direction, $b_{x}\left(b_{y}\right)$, as the standard deviation of the center-of-gravity, $s_{x}$, of the fluence distribution in the $x(y)$ direction, as measured from 100 shots, normalized to the average horizontal(vertical) beam radius, $\overline{2 \sigma_{x(y)}}$,

$$
b_{x(y)}=\frac{s_{x(y)}}{2 \sigma_{x(y)}} .
$$

In Fig.3.7, we plot the measured horizontal (Fig.3.7a) and vertical (Fig.3.7b) pointing fluctuation (pointing instability) versus the pressure for different drive laser energies. It can be seen that the horizontal beam pointing fluctuation lies in the range of 5 to $10 \%$ and does not change significantly with pressure. The vertical beam pointing fluctuation is larger, on the order of 10 to $30 \%$. Yet, with increasing pressure, the beam pointing fluctuation decreases. If we combine these results with the divergence measurements, we find that the absolute pointing fluctuation (i.e., $\left.s_{x(y)}\right)$ is approximately constant and independent of pressure and energy. From this we conclude that the interplay of nonlinear dynamics and capillary waveguiding, providing a stable mode of propagation for the drive laser as described above, indeed provides mode stability over wider pressure and energy ranges as predicted [95].

The observed absolute value for vertical pointing fluctuations $(0.3 \mathrm{mrad})$ is about three times larger than the value for the horizontal fluctuation $(0.1 \mathrm{mrad})$. We attribute this again to the slightly elliptical shape of the input driving pulse. 

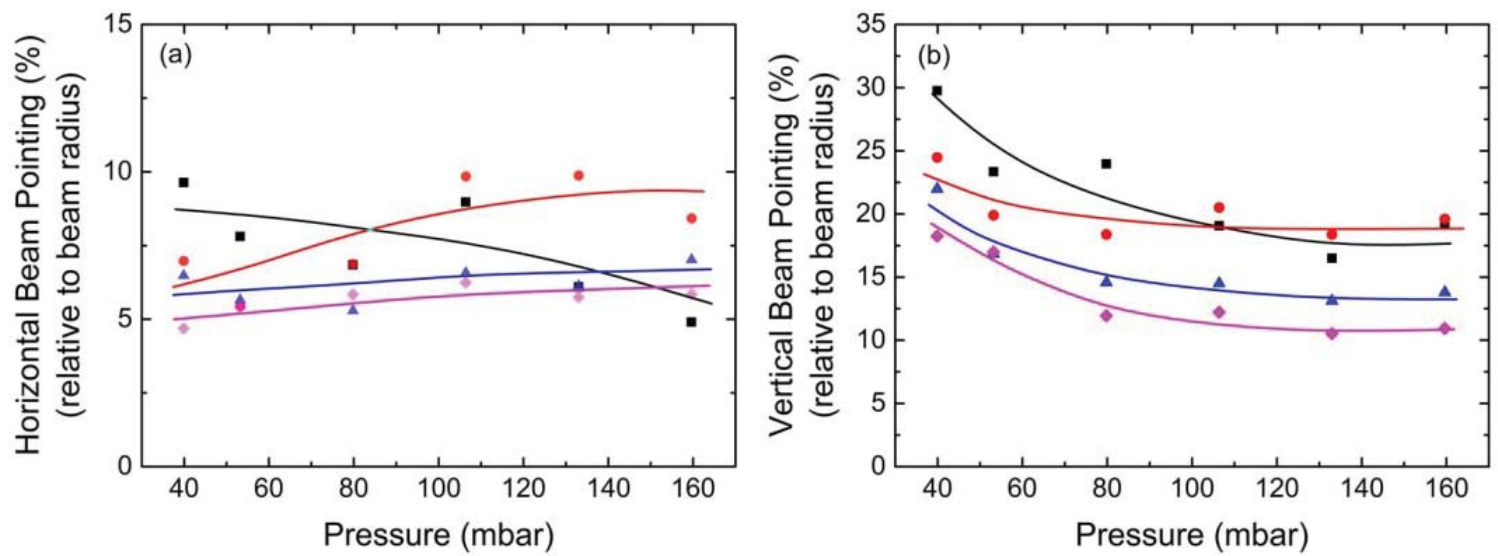

Figure 3.7: The high-harmonic beam pointing fluctuation relative to the average beam radius as a function of gas pressure for different drive laser intensities at $0.6 \mathrm{~mJ}$ (black squares), $0.8 \mathrm{~mJ}$ (red circles), $1.0 \mathrm{~mJ}$ (blue triangles), and $1.1 \mathrm{~mJ}$ (purple diamonds): (a) for the horizontal direction and (b) for the vertical direction.

In summary, high-harmonic generation in our gas-filled capillary achieves a highest $\mathrm{HH}$ output energy of $1.2 \mathrm{~nJ}$ at a drive energy of $1 \mathrm{~mJ}$ and a gas pressure of 53 mbar. At these conditions, we measure the following beam parameters: an energy jitter of $18 \%$, a divergence of $1.5 \mathrm{mrad}(x)$ and $1.6 \mathrm{mrad}(y)$, and a beam pointing fluctuation of $6 \%(x)$ and $17 \%(y)$. In comparison, non-waveguided HHG in gas cells showed an energy jitter of $6 \%$, a divergence of $0.7 \mathrm{mrad}(\mathrm{x})$ and $0.2 \mathrm{mrad}(\mathrm{y})$, and a beam pointing fluctuation of $4 \%$ ( $\mathrm{x}$ and $\mathrm{y}$ ) others [87], while HHG in gas jets show a somewhat higher energy fluctuation compared to HHG in gas cells [105]. In that case the origin of beam pointing and energy jitter was reported as possibly related to drive laser instabilities. Our result shows higher HH output fluctuations although our drive laser fluctuations are comparable with what was reported for the non-waveguided case. This raises the question whether the capillary amplifies the drive laser pulse fluctuations and correlations are present between the fluctuations in the driving laser pulse at the input of the capillary and the fluctuations in the highharmonic output beam.

\subsection{Correlation measurement of beam parameters between driver laser and $\mathrm{HH}$}

To investigate the influence of drive laser beam fluctuations on $\mathrm{HH}$ beam fluctuations, we make use of the single-shot drive laser beam profiles that were simultaneously recorded with the single-shot high-harmonic beam profiles. To reveal a possible correlation between the two, we compared the fluctuations in the center-of-gravity for the two beams (Fig. 3.8), the HH output energy versus the center-of-gravity of the drive laser beam (Fig. 3.9) and the energy in the pulses in 
the two beams (Fig. 3.10). To quantify the strength of a possible correlation, we calculate the Pearson's correlation coefficient, $\rho$, between two data sets A and B defined by [106]

$$
\rho(A, B)=\frac{\sum(a-\bar{a})(b-\bar{b})}{\sqrt{\sum(a-\bar{a})^{2} \sum(b-\bar{b})^{2}}},
$$

where $a(b)$ and $\bar{a}(\bar{b})$ are the elements of and average for dataset A (B). Here, $\rho=$ $1(-1)$ indicates a maximum correlation (anti-correlation) and $\rho=0$ indicates the absence of any correlation, i.e., mutually independent fluctuations.

A typical example of the center of gravity of the $\mathrm{HH}$ beam vs. the center of gravity of the drive laser beam is shown in Fig. 3.8, for each of the 100 single shots at a drive laser energy of $1.0 \mathrm{~mJ}$ and at 53 mbar Ar gas pressure, which corresponds to the highest HH output measured. The distributions shown in Fig. 3.8 are, on a first glance, uncorrelated. Indeed, the correlation coefficients are found to be close to zero; 0.07 and -0.11 for horizontal and vertical fluctuations, respectively. The correlation plots between mutually orthogonal directions [horizontal (vertical) drive laser fluctuations vs. vertical (horizontal) HH fluctuations] are not shown here, because the results are similar, i.e., a correlation of 0.09 for horizontal drive laser beam and vertical HH fluctuations, and a correlation of 0.08 for vertical drive laser beam and horizontal HH fluctuations. The weak beam pointing correlation between the drive laser and $\mathrm{HH}$ output beams indicates that drive laser beam pointing fluctuation is not a significant factor for $\mathrm{HH}$ beam pointing fluctuation in a waveguided geometry.
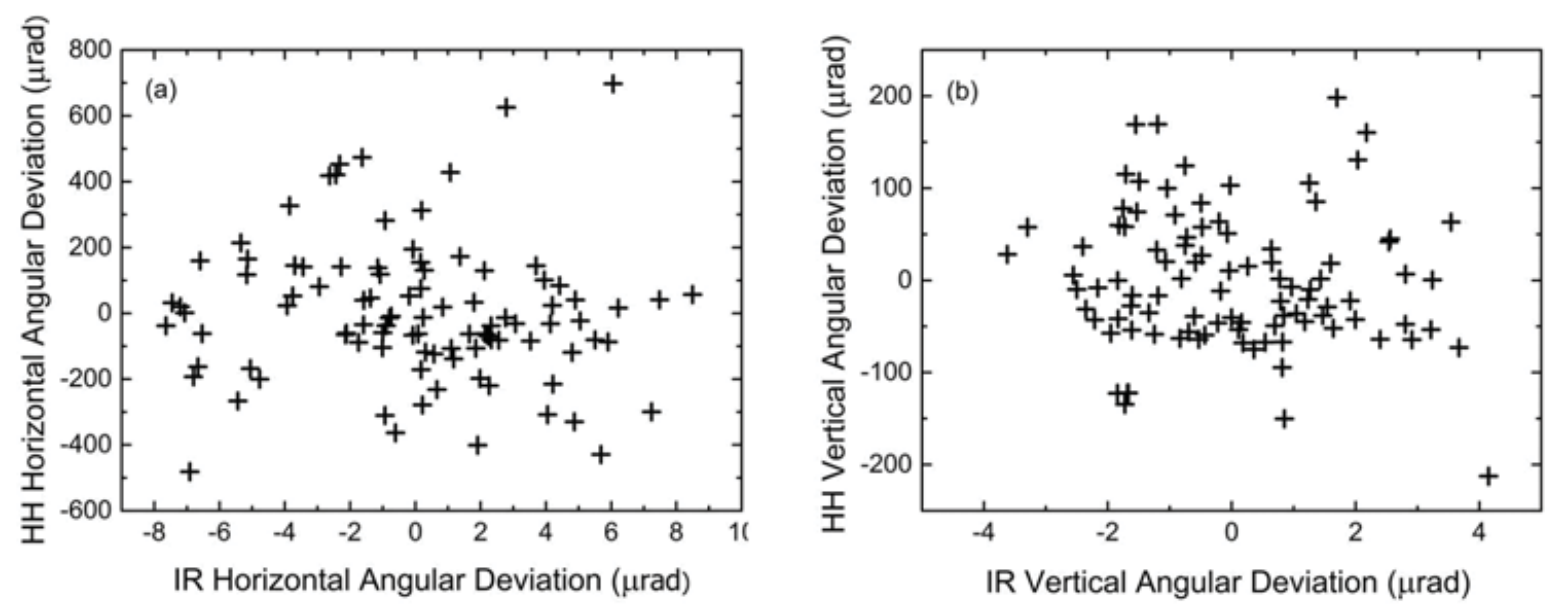

Figure 3.8: Correlation plot of 100 single-shot measurements of the deviation of the HH beam center of gravity from its average versus the drive laser center of gravity from its average for (a) both horizontal and (b) both vertical fluctuations; the drive laser energy is $1.0 \mathrm{~mJ}$ and the Ar gas pressure is 53 mbar. The correlation coefficients are $-0.07(a)$ and $-0.11(b)$, respectively, too small for indicating any correlation with statistical significance. 
In Fig. 3.9 we show the measured HH output energy as a function of the IR beam pointing for 100 single shots. The correlation coefficients are found to be 0.03 and 0.05 for horizontal and vertical pointing fluctuations, respectively. This means again that there is only a weak correlation between the $\mathrm{HH}$ output energy and the drive laser beam pointing.
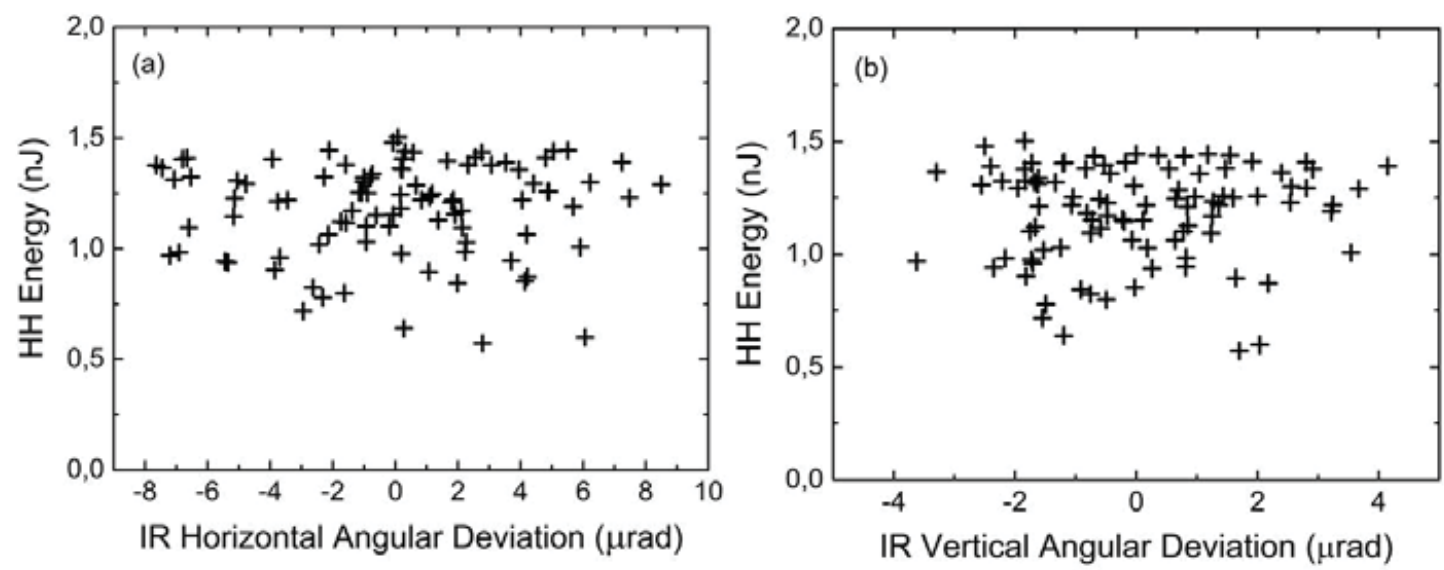

Figure 3.9: Energy in the HH beam versus the horizontal (a) and vertical (b) deviation of the IR beam center of gravity for 100 single shots. The drive laser energy is $1.0 \mathrm{~mJ}$ and the Ar gas pressure is 53 mbar. The correlation coefficients are 0.03 (a) and 0.05 (b), respectively.

Besides directional fluctuations, there is another type of drive laser instability that may cause HH energy fluctuations, which is fluctuation of the drive pulse energy. The presence of such effect should be visible as a correlation between drive laser pulse energy and $\mathrm{HH}$ output energy fluctuations. For an analysis, we have plotted the pulse energy of 100 single HH output pulses vs. the energy of the corresponding drive pulses, as seen in the example of Fig. 3.10. The correlation coefficient obtained from the data is close to zero again $(\rho=0.11)$. We conclude that there are only weak correlations between drive energy and HH energy fluctuations.

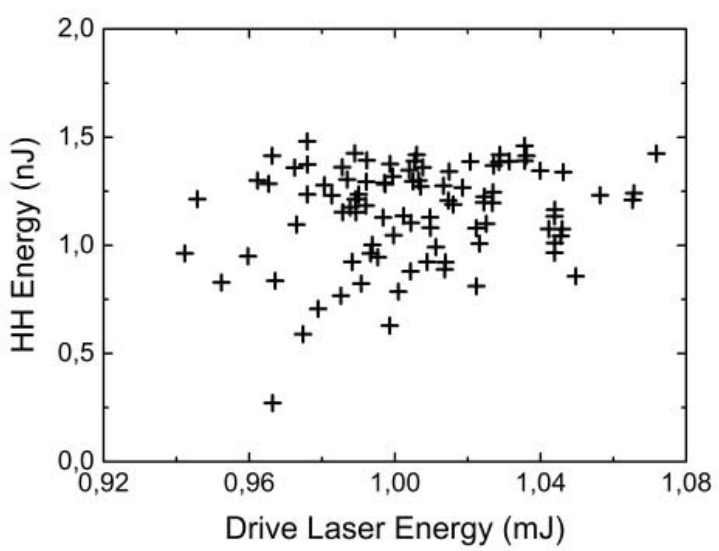

Figure 3.10: The HH beam energy versus the drive laser energy for 100 single shot. The drive laser energy is $1.0 \mathrm{~mJ}$ and the Ar gas pressure is 53 mbar. The correlation coefficient obtained from the data is 0.11 . 
As a final check for externally induced fluctuations of the $\mathrm{HH}$ output we investigated whether there are any instabilities introduced by the capillary. Although the capillary is firmly mounted at an optical table, it might be possible that small turbulences in the gas flow cause small fluctuations in the refractive index of the gas. Similarly, small acoustic perturbations may be guided from the vacuum pump system to the capillary. If significant, these effects should become visible as increased pointing and energy fluctuations of the drive laser behind the capillary. The data on the drive laser presented above shows that the beam pointing and pulse energy fluctuation before entering the capillary are in the range of $0.3-0.5 \%$ and $0.5 \%$, respectively. We have measured the drive laser pointing and energy fluctuations behind the capillary, with the gas flow on and off, and with the vacuum pumps on and off. We observe that there is no difference in fluctuations, with the gas flow and vacuum pumps on or off. However, we still measure that the pointing and pulse energy fluctuations increase to about $3 \%$ for the transmitted drive laser pulse behind the capillary.

The correlation measurements show that there is only a weak correlation between the fluctuations in the drive laser beam and high-harmonic beam. Still, we observe higher fluctuations in the transmitted drive laser beam, which do not seem to depend on the gas flow nor on acoustic perturbations from the vacuum pumps. We therefore conclude that the increase in fluctuations in the transmitted beam are due to fluctuations in the coupling of the drive laser beam into the capillary caused by a combination of a slight elliptical shape, a slight off-unity beam parameter and the energy and pointing fluctuations of the driving laser pulse. The weak correlation between the drive laser beam and the $\mathrm{HH}$ output beam fluctuations indicates a decoupling between these parameters. A possible explanation could be the observation that the most intense part of the drive laser pulse is very robust against variations in input pulse energy and pressure inside the capillary [95]. If this part of the drive laser pulse is dominantly responsible for the high-harmonic generation, a decoupling between the fluctuations in the drive laser pulse and in the generated higher harmonics can be expected. However, to confirm this a full simulation that includes the highly dynamical drive laser pulse propagation as well as the highharmonic generation process is required. Such a model is currently under development [107]. Another explanation could be that the propagation through the capillary introduces a randomization that removes the correlation. For example, local fluctuations in gas density result in local electron density variation and this affects both the modal spatial and phase distribution. However, a detailed investigation into this is beyond the scope of the present investigation. 


\subsection{Summary and conclusions}

In this chapter we present what is to our knowledge the first single-shot analysis of the output beam properties and stability in waveguided high-harmonic generation (HHG). The experiments are carried out in a thin waveguiding capillary of standard radius ( $75 \mu \mathrm{m}$ inner radius) filled with Ar gas, which yields a maximum $\mathrm{HH}$ pulse energy of $1.2 \mathrm{~nJ}$ in the range between $29 \mathrm{~nm}$ and $52 \mathrm{~nm}\left(15^{\text {th }}\right.$ and $27^{\text {th }}$ harmonic of a Ti:Sapphire laser) at a drive pulse energy of $1 \mathrm{~mJ}$ at $40 \mathrm{fs}$ drive pulse duration. We experimentally characterize the strength of various types of fluctuations in the highharmonic output, i.e., fluctuations in beam shape, beam pointing, and $\mathrm{HH}$ pulse energy vs. the gas pressure and drive laser pulse energy. We attribute the observed effects to ionization- induced nonlinear mode mixing and spectral broadening of the drive laser inside the capillary waveguide. In single-shot correlation measurements we observe a weak correlation between drive laser pointing or energy fluctuations with pointing or energy fluctuations in the $\mathrm{HH}$ output. Our investigations show that in waveguided HHG it is essential to have a very stable drive laser beam with very good beam quality and at the same time apply appropriate pressure and drive laser pulse energy for achieving the best compromise between a high beam stability and a maximum output pulse energy. 


\section{4 \\ Upscaling drive energy and waveguide dimensions for enhanced high-harmonic yield}

\subsection{Introduction}

Waveguide capillaries currently used for high-harmonic generation (HHG) [108] typically have a small inner radius of $75 \mu \mathrm{m}$ [86] and are driven with laser pulse of a few hundred $\mu \mathrm{J}$, resulting in high-harmonic outputs with pulse energies of several $\mathrm{pJ}$ up to a nJ. In order to increase the output, it seems straightforward to apply drive pulses with higher energy. However, such simple scaling up of the output via increasing the input is known to fail for the following reasons. At higher input pulse energy, the drive intensity becomes higher which will increase the degree of ionization. This will lead to a change in refractive index and thereby induce a loss of phase matching. Ionization also reduces the density of neutral atoms available to provide for high-harmonic generation which reduces the output further. This would hinder the growth of the $\mathrm{HH}$ output vs the interaction length [109]. Instead, nonlinear propagation effects for the drive laser would occur which are difficult to predict $[94,95,110]$ and reduce the spatial coherence of the output [111].

A potential solution to these problems at elevated drive pulse energies might be to increase the radius of the capillary with the drive energy. With an increased radius, while mode-matching the drive laser beam to the lowest order waveguiding capillary mode [77], the focal beam size will become larger as well. Since the drive intensity scales inversely proportional with the focal beam size, the increase of input pulse energy will maintain the drive intensity such that the ionization can be kept low and ionization induced phase-mismatch and defocusing can be mitigated. In this way, it might become possible to generate and phase-match the high-harmonics over a larger focal beam size and a longer interaction length for increasing the highharmonic output.

Here we theoretically and experimentally investigate the scale up of the highharmonic output based on this approach. In Section 4.2, we develop a model which calculates the build-up of the high-harmonic output pulse energy in an Ar-filled capillary taking into account spatiotemporal phase matching and also reabsorption of 
generated harmonic radiation. With the model, we calculate the relative yield of high-harmonic energy when we scale up the radius of the capillary with the drive energy. We also calculate the relative yield as a function of gas pressure and interaction length of the capillary. These results are presented in Section 4.3. Next, we describe a new experimental setup which enables the generation and characterization of high-harmonic generation in a gas-filled capillary with a large radius of $254 \mu \mathrm{m}$ in Section 4.4. With the new setup, we perform single-shot measurements as a function of gas pressure using two capillaries with interaction lengths of $37 \mathrm{~mm}$ and $220 \mathrm{~mm}$. We compare the experimental values for the highharmonic pulse energy with the theoretical values obtained with our model in Section 4.5. Finally, we conclude in Sect.4.6 with the major theoretical and experimental results and findings.

\subsection{Phase-matching model}

As we discussed previously (recall Section 2.3), efficient generation of highharmonics requires phase matching between the drive laser and the generated harmonics. When the phase matching condition is fulfilled, the high-harmonics from single atoms add up coherently upon co-propagation with the drive laser pulse. Here, where we consider the case of high-harmonic generation from neutral atoms inside a hollow waveguiding capillary, the mismatch in wave vectors, $\Delta k_{q}$, can be calculated as [54]

$\Delta k_{q}=\frac{2 \pi q}{\lambda}(1-\eta) \frac{P}{P_{a t m}} \Delta n-\frac{P}{P_{a t m}} \eta N_{a t m} r_{e} \lambda\left[\frac{q^{2}-1}{q}\right]-\frac{\mu_{l m}^{2} c}{2 a^{2} \omega}\left[\frac{q^{2}-1}{q}\right]$.

Here $\lambda$ is the vacuum wavelength of the drive laser, $q$ is the harmonic order, $\eta$ is the fraction of free electrons with respect to the neutral atom number density, $P$ is the gas pressure, and $P_{a t m}$ is the atmospheric pressure, $\Delta n$ is the difference between the refractive index of the neutral gas for the drive laser wavelength, $n(\lambda)$, and the index at $q^{\text {th }}$ harmonic wavelength, $n(\lambda / q)$, at atmospheric pressure, $N_{a t m}$ is the atomic number density at atmospheric pressure, $r_{e}$ is the classical electron radius, $\mu_{l m}$ is the $m^{\text {th }}$ root of the $l^{\text {th }}$ order Bessel function, and $a$ is the capillary radius.

In Eq.4.1, the first term on the rhs is due to dispersion of the neutral atoms, the second is due to the dispersion of free electrons and the last term is due to dispersion of the hollow capillary. For simplicity we assume that all of the drive pulse energy is propagating in the fundamental mode of the capillary, i.e., we set $l=1$ and $m=0$ which yields $\mu_{1 m}=2.405$. This can be approximately achieved in an experiment with an appropriate mode matching [77]. We also neglect the intensity dependent phase lag of harmonics, specifically for the so-called long electron trajectories which is 
caused by the intensity dependence of the time interval between the moment of ionization and recombination. Here, this approximation is justified because in a capillary the short trajectories are mainly contributing [112] to the $\mathrm{HH}$ output and these trajectories only show a weak intensity dependence.

When $\Delta k_{q}$ in eq. 4.1 becomes zero, in an analysis based on one-dimensional coupled-wave equations [113], the high-harmonic output power and intensity grows quadratically with the interaction length since the high-harmonic field grows linearly with the propagation length. However, in practice, reabsorption of the generated harmonics by the gas medium will impose a limit to the harmonic output. Even when phase matching is fulfilled perfectly well, i.e., $\Delta k_{q}=0$, the growth of the high-harmonic output vs. interaction length will terminate when interaction length is longer than a few absorption lengths, $L_{a b s}$. Taking both effects into account, i.e, phase-matching and absorption, the coupled-wave equations predict a build-up of the harmonic pulse energy, $N_{q}$, proportional to [104]

$$
N_{q} \sim N_{a}^{2} A_{q}^{2}\left[\frac{1+e^{-2 \alpha L_{m}}-2 \cos \left(\Delta k_{q} L_{m}\right) e^{-\alpha L_{m}}}{\alpha^{2}+\Delta k_{q}^{2}}\right],
$$

where $\mathrm{N}_{\mathrm{a}}$ is the neutral gas density, $L_{m}$ is the length of the capillary that is filled with the gas medium, $\alpha=1 /\left(2 L_{a b s}\right)$ is the HH field absorption coefficient, $L_{a b s}$ the power or intensity absorption length, and where $A_{q}$ is the amplitude of the singleatom response, i.e., the induced nonlinear polarization at the $q^{\text {th }}$ harmonic frequency. To predict the absolute value for the intensity generated at a particular harmonic, an extensive modeling [114] is required that includes a numerical solution of the timedependent Schrödinger equation to calculate the single atom response. For simplicity, we set $A_{q}$ to an approximate value of $(1-\eta)\left|E_{d}\right|^{5}[104,114]$, where $E_{d}$ is the modulus of the instantaneous electric field of the drive laser ( $\eta$ is the fraction of free electrons with respect to the neutral atom number density as in Eq.4.1). This approximation includes the ground state depletion by ionization as well as the intensity dependence of the $q^{\text {th }}$ harmonic response in the strong-field regime [104] as compared to the perturbative regime where $A_{q}$ is proportional to $\left|E_{d}\right|^{q}$. Although this approximation does not allow for the calculation of an absolute harmonic output, we note that it is sufficient for the scaling study undertaken here, for predicting the relative output for various experimental parameters.

To calculate the relative output, we first calculate the radial distribution of the ionization fraction as a function of time , $\psi(r, t)$, using the Ammosov, Delone and Krainov (ADK) model [67] (recall Eq.2.3) that can be applied in the strong-field regime discussed here, where $r$ is the radial coordinate in the capillary and $t$ is time. Next, we determine the refractive indices of the neutral gas at the drive laser wavelength, $n(\lambda)$, and at high-harmonic wavelengths, $n(\lambda / q)$. For near infrared drive laser wavelengths as used here, the refractive index for $\mathrm{Ar}$ is available from direct 
measurements with a frequency comb [115]. For high-harmonic wavelengths in the XUV range, we make use of the complex refractive index as obtained via the following expression $[100,116]$ :

$$
n=1-\frac{N r_{e} \lambda^{2}\left(f_{1}+i f_{2}\right)}{2 \pi}
$$

where $f_{1}$ and $f_{2}$ are the real and imaginary parts of the atomic scattering factor. Henke et al. [100] provides the $f_{1}$ and $f_{2}$ data, where the $f_{2}$ values are calculated from measured photoabsorption spectra and $f_{1}$ values are calculated via KramersKronig relations. Although the $\mathrm{f}_{2}$ data from Henke et al. [100] range from $0.04 \mathrm{~nm}$ to $124 \mathrm{~nm}$, the $\mathrm{f}_{1}$ data are limited to wavelength below $40 \mathrm{~nm}$. We note that the wavelength range that is accessible with our detection system in the experiments extends up to $70 \mathrm{~nm}$ where no data for $\mathrm{f}_{1}$ are available from Henke et al. Hence, for the remaining wavelength range of interest, from $40 \mathrm{~nm}$ to $70 \mathrm{~nm}$, we use $f_{1}$ data provided by Chantler et al. [116].

Having obtained the intensity and time dependent ionization fraction and spectral variation of the refractive indices, we can now calculate the local wave vector mismatch, $\Delta k_{q}(r, t)$, from Eq.4.1. As a next step, we determine also the absorption length, $L_{a b s}$, of the harmonic $q$ from the atomic scattering factor, $\mathrm{f}_{2}$, again provided by Henke et al. [100], with the following equation:

$$
L_{a b s}=\frac{1}{2 r_{e} f_{2} \lambda_{q} \lambda N_{a}}
$$

where $N_{a}$ is the neutral gas density. Next, we use Eq. 4.2 to calculate for each radial position and each time in the drive laser pulse, the contribution to the output at a particular, single harmonic $q$. Finally, by integrating Eq. 4.2 over the pulse duration and the spatial cross-section area of the capillary, we obtain the total relative output pulse energy for each harmonic.

Note that due to the dependence of Eq.4.2 from $\Delta k(r, t)$, the largest contribution to this output is coming from spatial regions in the capillary and from time intervals where the generation is transiently phase matched, i.e., where $\Delta k_{q}=0$ because the ionization fraction, $\eta(r, t)$, passes through the required value. The derivation of relative $\mathrm{HH}$ output has relied, via application of the ADK model to determine $\eta(r, t)$, on the assumption that tunneling of electrons is the main mechanism of ionization. Usually this assumption is valid when the intensity is high enough, which is the case for most of the volume of the capillaries, considered here. Only very near the walls of the capillary, the intensities are insufficient for tunnel ionization. However, we expect that this will not noticeably affect the output calculation as (i) the ionization 
level will be too low for phase matching (i.e., large $\left|\Delta \mathrm{k}_{\mathrm{q}}\right|$ ) and (ii) the local field amplitude, $A_{q}$, is anyway too low to generate the desired harmonic.

Previously, others have devised various different models to describe phasematching in capillaries for calculating the buildup of harmonic field. A first type of approach is to use numerical propagation codes [15,16] which is, however, computationally rather costly. A second type of approach uses analytic calculations and includes the radial effects only as their average, thereby assuming, to our impression less realistic, a radially homogeneous ionization [12-14]. A third type of approach uses analytic calculations and takes into account radial temporal variation [117] but is computationally fast. Our model is identical to that approach, however, we vary different parameters and over a wider range. The model in [117] restricts itself to a capillary of a fixed diameter and calculates relative intensities of the harmonics for a number of different gases. In contrast, we use our model for looking into the relative total intensities at a single gas, but we vary the diameter and length of the capillary, as well as gas pressure and drive pulse energy.

Regarding the expected validity range of the described model, we look at the approximations made. Our model only considers the lowest order waveguide mode for the drive laser propagation throughout the capillary. We note that in an experiment, some energy will be coupled into higher order waveguide mode for any coupling of a Gaussian beam into the capillary. With ideal ratio of waist radius to capillary radius, $w / a=0.64$, there will still be some finite degree of excitation of higher order modes to be above 2\% [77]. For drive laser beams that are noncircular or show a beam parameter $\left(\mathrm{M}^{2}\right)$ larger than one, this incoupling into higher order modes will grow. Furthermore, our model assumes that the propagation of the drive laser intensity is linear, which means that the pulse peak intensity, temporal and spatial profile remain the same over the build-up length of the high-harmonic generation. In other words, we assume that nonlinear propagation effects such as ionization induced index changes and self-focusing and defocusing can be neglected.

In an experiment, the electron density will grow with the gas density, the laser intensity and pulse duration. We therefore expect that our model assumptions would hold only in a certain range of lower gas pressure and lower intensities for a thin capillary, and hold for correspondingly higher values in a thick capillary.

\subsection{Propagation model results}

In the following we use the model described above for an investigation on what role the most essential parameters, the gas pressure, $P$, the capillary radius, $a$, and the length of the medium, $L_{m}$, play for scaling up the $\mathrm{HH}$ output in a capillary with drive laser pulse energy. For specificity, and also for allowing a comparison with own experimental data, we base our calculations on the typical pulse energies that are 
routinely accessible to us. These are pulse energies of about $6 \mathrm{~mJ}$ (maximum laser specification is $8 \mathrm{~mJ}$ ), a pulse duration, $T_{\mathrm{p}}$, of $40 \mathrm{fs}$ (FWHM) and two capillary diameters. The first is a standard value of $150 \mu \mathrm{m}$ that is commonly used by other researchers. The second is a much larger diameter of $508 \mu \mathrm{m}$ (radius of $254 \mu \mathrm{m}$ ).

To illustrate which regions typically contribute to phase-matched $\mathrm{HH}$ generation we plot in Fig. 4.1 the wave vector mismatch, $\Delta k_{q}$, as a function of radial position, $r$ and time, $t$ for a drive pulse $\left(6 \mathrm{~mJ}, T_{p}=40 \mathrm{fs}\right)$ for the $21^{\text {st }}$ harmonic $(q=21)$, at a pressure of $P=20$ mbar in a capillary with length $L_{m}=37 \mathrm{~mm}$. The center of the drive laser pulse in time is at $t=0$ and in radial position is at $r=0$. The solid white line indicates where full phase-matching is obtained. Around this line, a small volume around this region contributes to the HH output, i.e., $\left|\Delta_{q}\right|<75 \mathrm{~m}^{-1}$ for $L_{m}=$ $37 \mathrm{~mm}$ as defined by the FWHM of the phase matching curve. It can be seen at times earlier than $-15 \mathrm{fs}$, i.e., in the rising front of the laser pulse, where no phase matching occurs at any radial position since the ionization fraction is still too low. Thereafter, a sufficient ionization fraction is reached in the center of the capillary (on axis) where the intensity is highest, such that HHG becomes phase matched on axis. At later times, the ionization fraction becomes too high and the harmonic is no longer phase matched on axis. At the same time, the off-axis ionization fraction is increased as well and phase matching becomes fulfilled at $r \neq 0$. It can be seen that phase matching is obtained further from axis as time progresses. Finally, at times later than $10 \mathrm{fs}$ where the laser power is dropping, there is no significant increase of the ionization and phase matching remains fulfilled for a certain radius, in the shown example at around $90 \mu \mathrm{m}$. Fig. 3.1 shows clearly that phase matching in a waveguide capillary is for most radii a transient situation, dynamic spatio-temporal effect, to be taken into consideration when calculating the total high-harmonic output.

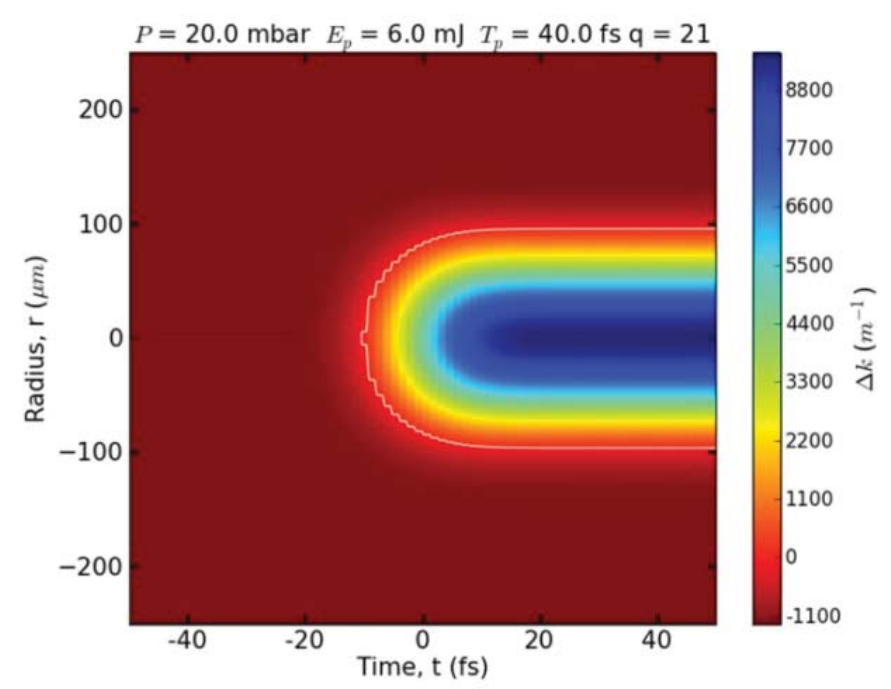

Figure 4.1: Wave vector mismatch $\Delta k$ as a function of radial position and time for a gas pressure, $P=$ 20 mbar, drive laser energy of $\mathrm{E}_{\mathrm{p}}=6.0 \mathrm{~mJ}$, pulse duration $(\mathrm{FWHM})$ of $T_{p}=40 \mathrm{fs}$, and harmonic order of $q=21$. 
To systematically search for the parameters that promise to yield highest output, we calculate the relative harmonic pulse energy for varying parameters. Here we start with the $21^{\text {st }}$ harmonic order, which we observed to be the strongest harmonic order in the previous measured spectrum (Fig. 3.2 (c)). The first parameter we vary is the radius, $a$, of the capillary. Fig. 4.2 shows how the relative output at the $21^{\text {st }}$ harmonic $\left(\lambda_{\mathrm{q}=21}=38.1 \mathrm{~nm}\right)$ varies with the radius, $a$, for six different pulse energies between $0.3 \mathrm{~mJ}$ and $8 \mathrm{~mJ}$, while keeping the other parameters constant. Fig. 4.2 shows that the relative output at the considered harmonic increases from a value of 0.33 to 33 when the pulse energy is increased from $0.3 \mathrm{~mJ}$ to $8 \mathrm{~mJ}$. This corresponds to an increase in $\mathrm{HH}$ output by a factor of 100 achieved with an increase of drive laser energy by a factor of 27 . Observing that the ratio of the two values, $\approx 3.7(100 / 27)$ is above unity appears to suggest that not only the output but also the conversion efficiency may be readily increased by approximately a factor of 3 simply by increasing the capillary radius with a similar factor $3.3(250 \mu \mathrm{m} / 75 \mu \mathrm{m})$ and the upscaling the drive laser pulse energy from $0.3 \mathrm{~mJ}$ to $8 \mathrm{~mJ}$. However, we note that this is not necessarily the case as the optimum performance also depends on pressure and interaction length.

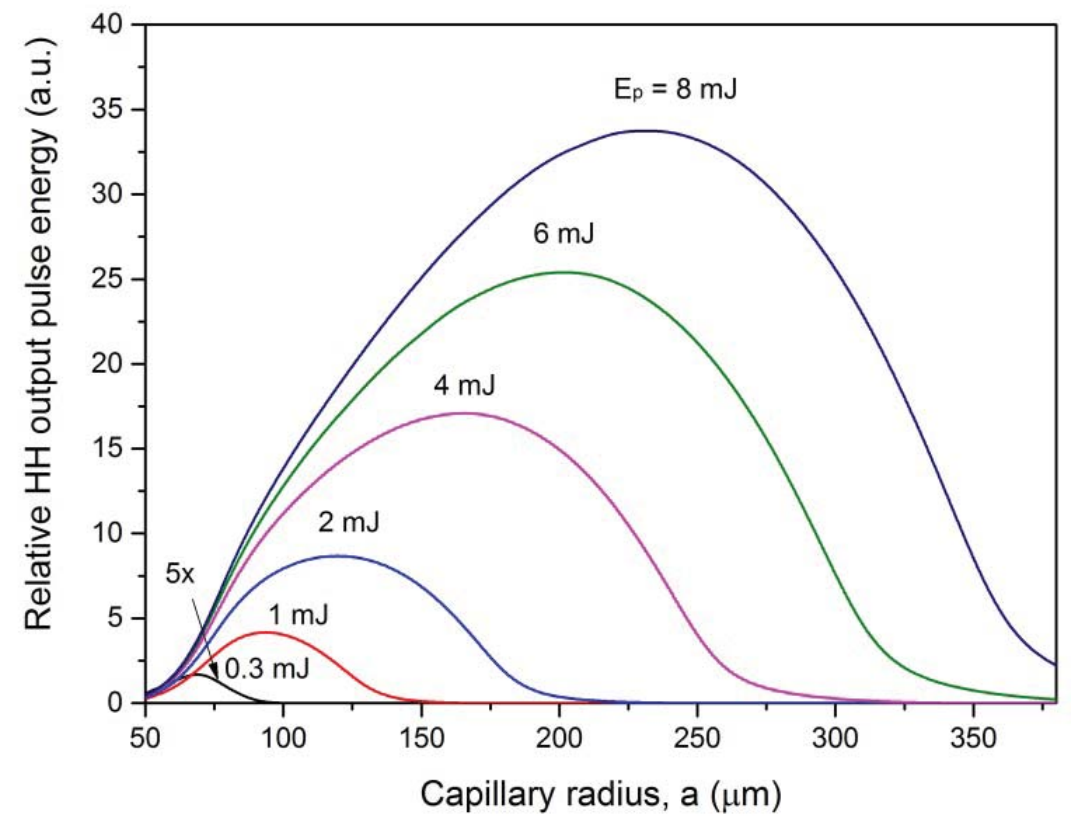

Figure 4.2: Scaling of the relative HH output at harmonic $q=21$ as a function of capillary radius, $a$, for various drive laser pulse energies, $E_{p}$, and a fixed pulse duration, $T_{p}=40 \mathrm{fs}$. The assumed Ar pressure here is $P=20$ mbar and the length of the capillary, $L_{m}=37 \mathrm{~mm}$

These additional dependencies calculated as output vs. pressure and interaction length are shown in Figs. 4.3 and 4.4, respectively where we have chosen a radius near the optimum values as predicted by Fig. 4.2. Figure 4.3 shows the HH output as 
a function of pressure, again for six same values of pulse energies between $0.3 \mathrm{~mJ}$ and $8 \mathrm{~mJ}$. It can be seen that, at $8 \mathrm{~mJ}$, the $\mathrm{HH}$ output initially increases sharply with pressure towards a maximum output at a relatively low pressure around 4 mbar. For higher pressures, the output drops slightly (by 5\%) and assumes an almost constant value above 10 mbar. This means that the maximum $\mathrm{HH}$ output for $8 \mathrm{~mJ}$ pulses is slightly underestimated in Fig.4.2. We observe a similar pressure dependence of the $\mathrm{HH}$ output for $E_{p}=6 \mathrm{~mJ}$ and $4 \mathrm{~mJ}$, i.e., a steep rise followed by a maximum at relatively low pressure. However, at lower drive energy i.e., at $E_{p}=0.3 \mathrm{~mJ}, a=75$ $\mu \mathrm{m}$ and $L_{m}=37 \mathrm{~mm}$, which is plotted as the black curve in Fig.4.3, the maximum output is reached only at a rather higher gas pressure, above approximately $30 \mathrm{mbar}$. The shift of the harmonic output peak to a higher pressure for lower drive energy can be understood via a phase matching argument using Eq.3.1 as follows. At lower drive energy, in order to reach appropriate intensities for high-harmonic generation, i.e., an appropriate value for $A_{q}$ on eq.3.1, the optimum capillary radius is smaller. A thin capillary, however, imposes higher waveguide dispersion via the last term in eq.3.1. Thus, a higher pressure is required in the first term for reaching $\Delta k_{q}=0$.

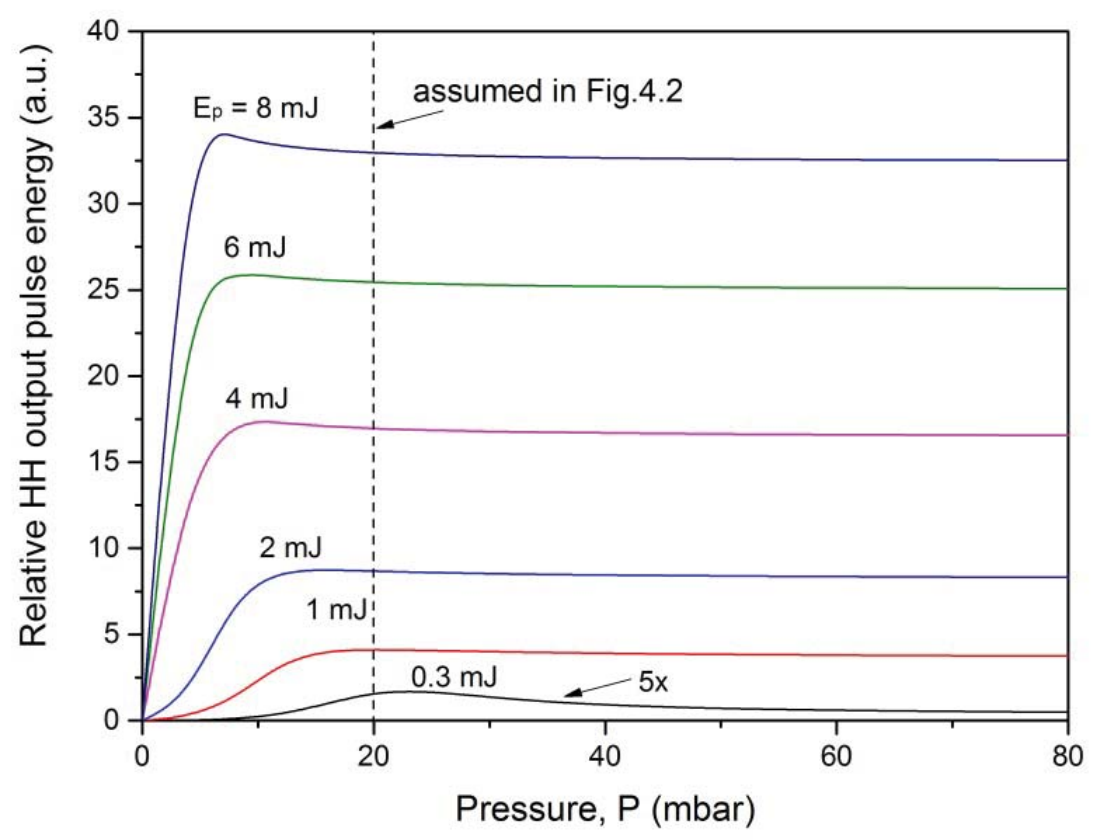

Figure 4.3: Scaling of the relative HH output at the $21^{\text {st }}$ harmonic $(\mathrm{q}=21)$ as a function of gas pressure, $P$, for various drive laser pulse energies, $E_{p}$, and a fixed pulse duration, $T_{p}=40 \mathrm{fs}$. The assumed length of the capillary is $L_{m}=37 \mathrm{~mm}$.

Finally, using the optimum pressures predicted by Fig.4.3, we investigated the dependence of the $\mathrm{HH}$ output as a function of the interaction length. These data are shown in Fig.4.4 and serve to find the optimum interaction length for the various pulse energies between $0.3 \mathrm{~mJ}$ and $8 \mathrm{~mJ}$. It can be seen that for all cases there is an initial increase with interaction length, but then the HH output levels off after $L_{m}=$ 
$30 \mathrm{~mm}$ for $8 \mathrm{~mJ}$, and after $L_{m}=20 \mathrm{~mm}$ for $0.3 \mathrm{~mJ}$. These figures indicate when the length of the capillary is at an optimum value, i.e. longer than $20 \mathrm{~mm}$ for $E_{p}=0.3$ $\mathrm{mJ}$ and longer than $30 \mathrm{~mm}$ for $E_{p}=8.0 \mathrm{~mJ}$.

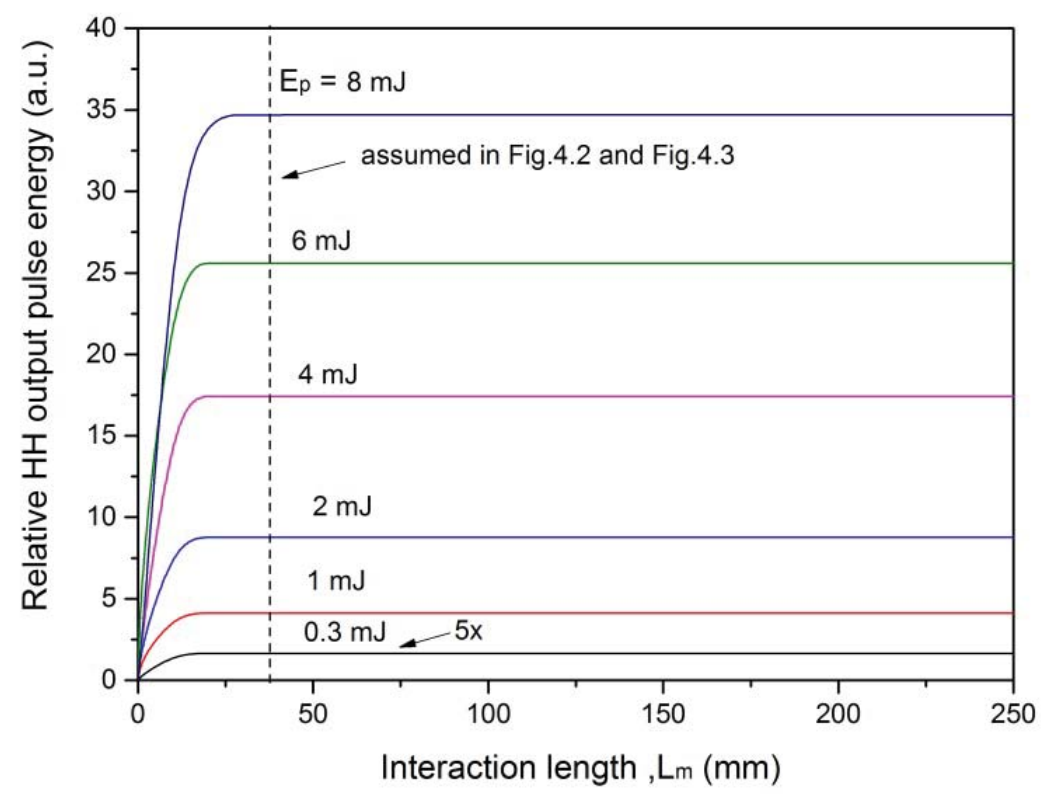

Figure 4.4: Scaling of the relative HH output at harmonic $q=21$ as a function of the interaction length, $L_{m}$, for various drive laser pulse energies $E_{p}$ and a fixed pulse duration, $T_{p}=40 \mathrm{fs}$.

After having shown the dependence of the HH output in a separate variation of scaling parameters $\left(a, P, L_{m}\right)$ we have also calculated the maximum output for a simultaneous variation of all parameters. In addition, to lift the restriction to a single harmonic, and enable a better comparison with own experimental data, we have calculated the optimum output for a range of harmonics, by summing their output energies. We include the spectral range from the $13^{\text {rd }}$ to the $29^{\text {th }}$ harmonic because this is the range that is covered with our detection system in the experiments. The results are summarized in Fig 4.5 and the optimum values of the parameters (capillary radius, pressure and interaction length) for maximizing the output pulse energy are given in Tab.4.1. 


\begin{tabular}{|c|c|c|c|c|c|c|}
\hline $\begin{array}{c}\mathrm{E}_{\mathrm{p}} \\
(\mathrm{mJ})\end{array}$ & 0.3 & 1 & 2 & 4 & 6 & 8 \\
\hline$q$ & \multicolumn{6}{|c|}{$a(\mu \mathrm{m}) / P(\mathrm{mbar}) / L_{m}(\mathrm{~mm})$} \\
\hline 13 & $60 / 10 / 30$ & $100 / 10 / 50$ & $125 / 10 / 50$ & $175 / 20 / 50$ & $200 / 10 / 60$ & $250 / 25 / 60$ \\
\hline 15 & $60 / 20 / 20$ & $100 / 20 / 20$ & $125 / 10 / 20$ & $175 / 20 / 20$ & $200 / 10 / 30$ & $250 / 7 / 30$ \\
\hline 17 & $70 / 20 / 20$ & $100 / 20 / 20$ & $125 / 20 / 25$ & $175 / 20 / 30$ & $200 / 10 / 30$ & $250 / 6 / 30$ \\
\hline 19 & $70 / 20 / 30$ & $100 / 20 / 30$ & $125 / 20 / 35$ & $175 / 20 / 45$ & $200 / 10 / 50$ & $250 / 12 / 50$ \\
\hline 21 & $70 / 20 / 20$ & $100 / 6 / 20$ & $125 / 7 / 20$ & $175 / 10 / 20$ & $200 / 15 / 30$ & $250 / 5 / 30$ \\
\hline 23 & $70 / 25 / 20$ & $100 / 20 / 20$ & $125 / 15 / 20$ & $175 / 10 / 20$ & $200 / 8 / 30$ & $250 / 7 / 30$ \\
\hline 25 & $70 / 25 / 20$ & $100 / 15 / 20$ & $125 / 10 / 30$ & $175 / 7 / 40$ & $200 / 6 / 50$ & $250 / 4 / 70$ \\
\hline 27 & $60 / 30 / 30$ & $100 / 20 / 30$ & $125 / 15 / 30$ & $175 / 15 / 50$ & $200 / 30 / 40$ & $250 / 20 / 50$ \\
\hline 29 & $60 / 40 / 50$ & $100 / 40 / 30$ & $125 / 40 / 40$ & $175 / 40 / 40$ & $200 / 40 / 40$ & $250 / 40 / 40$ \\
\hline
\end{tabular}

Table 4.1: The optimum combination of capillary radius $(a)$, gas pressure $(P)$ and capillary length $\left(L_{m}\right)$ that theoretically maximize the output pulse energy as a function of harmonic order $(q=13,15,17,19$, $21,23,25,27,29)$ for a range of drive energies, $E_{p}=0.3,1,2,4,6,8 \mathrm{~mJ}$. 


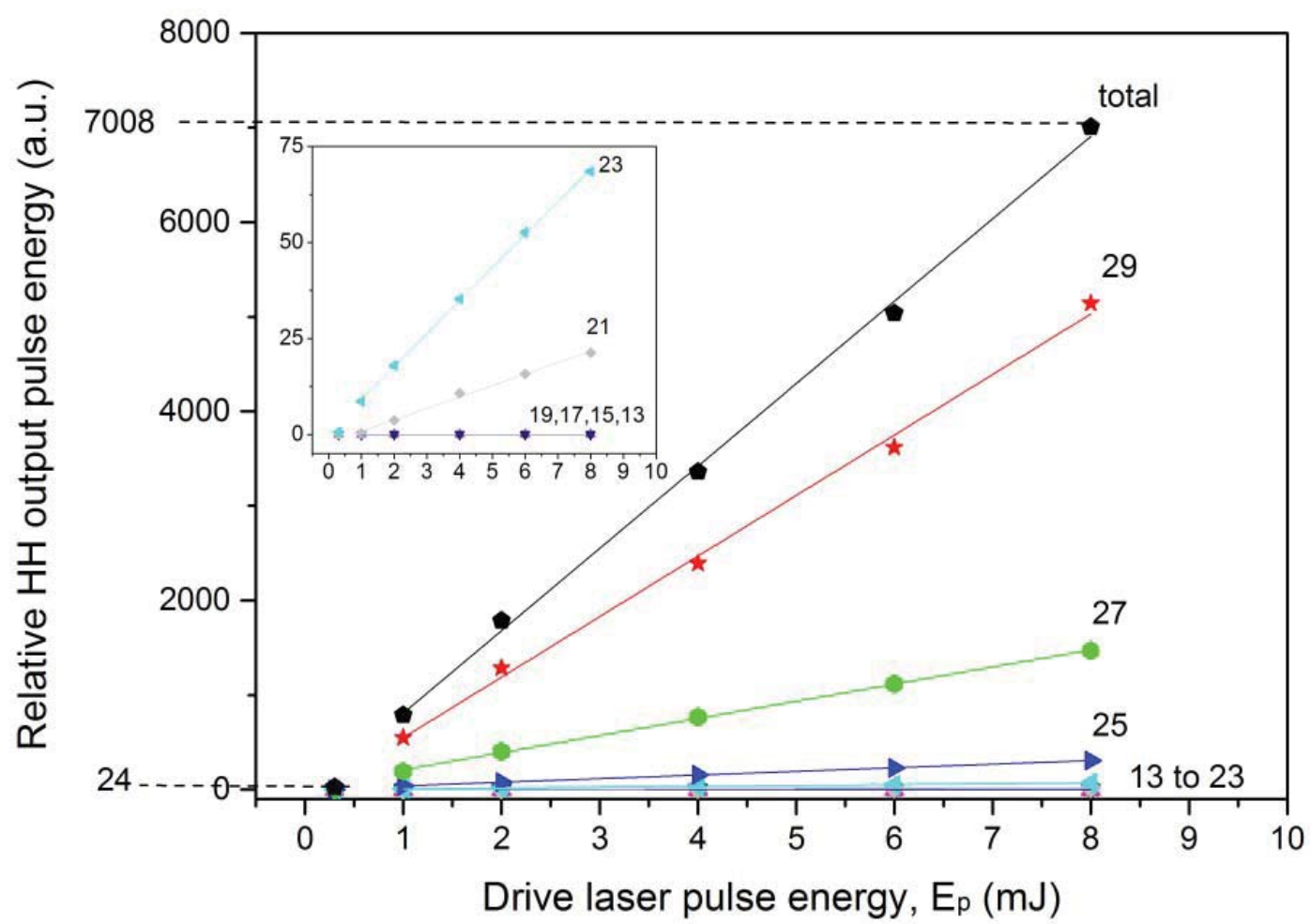

Figure 4.5: The HH output for a range of harmonics $(\mathrm{q}=13,15,17,19,21,23,25,27,29)$ and the summation (total) of harmonics as a function of drive laser pulse energy, $E_{p}$. The inset shows an enlarged view for the $\mathrm{HH}$ output of harmonics ranging from $13^{\text {th }}$ to $23^{\text {rd }}$.

From Fig.4.5, the total relative output increases from a value of 24 to 7008 when the pulse energy is increased from $0.3 \mathrm{~mJ}$ to $8 \mathrm{~mJ}$. These numbers differ from what we obtained earlier for simplified conditions (restriction to a specific harmonic order) and stepwise, separate variation of parameters. Here we find that the $\mathrm{HH}$ output increases by a factor of 290 achieved with an increase of drive laser energy by a factor of 27 which indicates a factor of 11 increasing conversion efficiency. However, from $1.0 \mathrm{~mJ}$ onwards, we observe that both the output at the individual harmonics and the total output (sum of all harmonics) scale up linearly with the drive energies. These observations indicate that above a certain drive energy threshold, $\mathrm{E}_{\text {th }}$ (between $0.3 \mathrm{~mJ}$ and $1.0 \mathrm{~mJ}$ ), the conversion efficiency remains the same and scales up linearly with the drive energy. Due to the unknown value of $\mathrm{E}_{\text {th }}$, the linear fittings for all data points shown in Fig. 4.5 begin from $1.0 \mathrm{~mJ}$ onwards.

In summary of Sect.4.2 and Sect.4.3, we have presented a simple model which calculates the build-up of high-harmonic energy in an Ar-filled capillary taking into account spatiotemporal phase matching and also reabsorption of generated harmonic radiation. We expect this model to be valid when the electron density is lower than the threshold given by Eq.3.2 such that nonlinear mode-coupling of the driving laser pulse remains weak. When this condition applies, the model predicts a scale up of the high-harmonic output when we scale up the radius of the capillary with the drive 
laser energy. In order to test the validity of the model, in the following section we describe an experiment to which the model can be compared.

We note that using a wider capillary for HHG requires the focusing length of the drive laser, $L_{f}$, to become longer for mode-matching the drive laser beam to the lowest order waveguide mode, i.e., $L_{f}=2.5 \mathrm{~m}$ when $a=250 \mu \mathrm{m}$. While applying higher drive laser energy (i.e., $E_{p}>6 \mathrm{~mJ}$ ) requires the drive laser to propagate at least nine-meter long in vacuum after the capillary to ensure that the drive laser beam has been diffracted sufficiently so that its fluence does not exceed the damage threshold of Al filters. Due to considerable effort and time required for designing and building such a large-size experimental setup, the experiment has been carried out for a constant drive energy $(6 \mathrm{~mJ})$ using a specific inner radius $(254 \mu \mathrm{m})$ for the capillary. This combination $(6 \mathrm{~mJ}, 254 \mu \mathrm{m})$ comprises of the maximally allowed drive energy and capillary radius for the experiment within our available lab space.

\subsection{Experimental setup}

We recall that the experimental setup described in the previous chapter (recall Section 3.2) that employing a thin capillary with $75 \mu \mathrm{m}$ inner radius is limited for driving HHG with varying IR pulse energy to values below $1.1 \mathrm{~mJ}$ because higher pulse energies impose various problems with ionization. Here, we are going to apply the full, available pulse energy of up to $6.0 \mathrm{~mJ}$ to drive HHG in a thick capillary of $254 \mu \mathrm{m}$ radius. For this purpose, we have constructed a new experimental setup as shown schematically in Fig. 4.6.

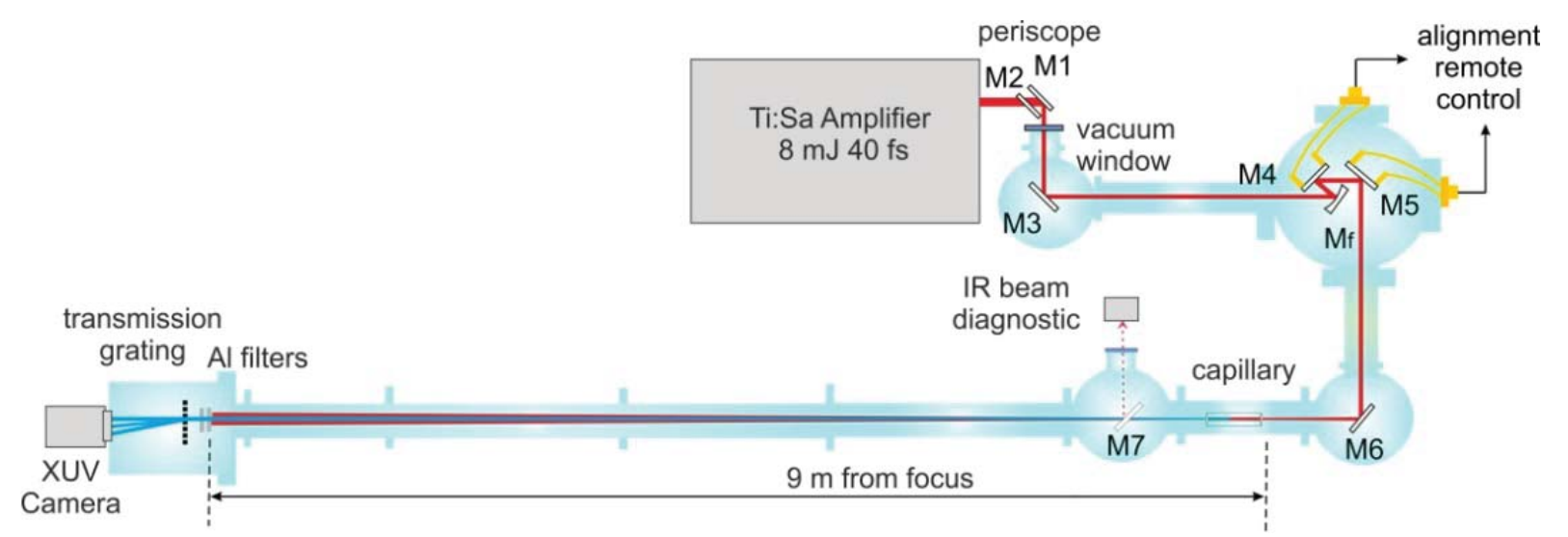

Figure 4.6: (a) Experimental setup of our high-harmonic generation source equipped with a gas-filled capillary with a radius of $254 \mu \mathrm{m}$.

To generate the drive laser pulses we employ a Ti:Sapphire infrared (IR) laser system (Legend Elite Duo HP USP, Coherent Inc.) with a repetition rate of $1 \mathrm{kHz}$ and a center wavelength of $795 \mathrm{~nm}$. The laser provided almost Fourier-limited pulses with 40 fs duration and a time bandwidth product (TBP) of 0.5 as measured 
with a home-built Grenouille [96]. To avoid self-phase modulation [97] of the drive laser beam as caused by propagation in air, the laser beam enters a vacuum chamber through a thin vacuum window which is placed as close as possible (near $50 \mathrm{~cm}$ ) behind the compressor grating. For matching the drive laser beam to the lowest order waveguiding mode for the thick capillary, a spherical mirror of $2.5 \mathrm{~m}$ focal length $\left(\mathrm{M}_{\mathrm{f}}\right)$ is used to focus the radiation into the capillary. The capillary is connected to the vacuum chamber at a distance of $4.0 \mathrm{~m}$ from the entrance window with the entrance of the capillary at the focal point of the spherical mirror.

In order to compensate astigmatism present in the drive laser beam, the spherical mirror $\left(\mathrm{M}_{\mathrm{f}}\right)$ is to be used under a non-normal angle calculated to be 14 degrees in the vertical incidence plane. We use a pair of mirrors (M1 and M2) before the vacuum system as a periscope to rotate the beam by 90 degrees. In this way, the spherical mirror can be placed under 14 degrees in the horizontal plane instead of vertical plane, which does not change the beam height. The corresponding calculations for astigmatism compensation can be found in Appendix A. With this arrangement, we measure the drive laser to be free of astigmatism, although there remains a slight ellipticity (1:2 aspect ratio) and a slightly off-unity beam parameter $\left(M_{x}^{2}=1.1 ; M_{y}^{2}=\right.$ $1.3)$.

Behind the spherical mirror, we place two mirrors (M3 and M4) which are remotely actuated to enable the alignment in terms of position and angle of the drive laser beam at the capillary entrance while the whole system is evacuated. The distance of the last mirror (M6) from the capillary entrance (focus point of the drive laser) is an important design parameter because it is required to keep the laser beam fluence incident on the M6 mirror below its damage threshold. For determining the maximum allowable fluence, we tested the M6 mirror damage threshold by placing the mirror at various positions from the capillary entrance while operating the drive laser at $6 \mathrm{~mJ}$ pulse energy. We found the minimum safe distance from the capillary entrance to be $85 \mathrm{~cm}$, and the M6 mirror is placed there.

For this setup, we have prepared two capillaries of the same radius, $a=254 \mu \mathrm{m}$, but with different lengths. The first capillary is of $67 \mathrm{~mm}$ long with the length and the slits manufactured to be the same as the thin $(a=75 \mu \mathrm{m})$ capillary used for the experiment described in the previous chapter (recall Fig.3.1). The interaction length of the capillary $\left(L_{m}=37 \mathrm{~mm}\right)$ capillary is equivalent to 0.4 times the Rayleigh length $\left(\mathrm{z}_{\mathrm{R}}=101 \mathrm{~mm}\right)$ for the focused beam. In order to extend the interaction length beyond the Rayleigh length, to allow for a larger volume of high-harmonic generation, we prepared a second capillary of $250 \mathrm{~mm}$ length in which the interaction length $\left(L_{m}=220 \mathrm{~mm}\right)$ is two-times the Rayleigh length.

We begin our HHG measurement (beam profile and spectra) with the first $\left(L_{m}=37\right.$ $\mathrm{mm})$ capillary installed into the setup. Upon completion of the measurement, we take the first capillary out from the setup and replace it with the second $\left(L_{m}=220\right.$ $\mathrm{mm})$ capillary. Due to the considerable difference in the length of the second 
capillary from the first one, some parts of the experimental setup are reconfigured to accommodate for the second capillary.

At two meter behind capillary, we place a $10 \%$ beamsplitter which can be moved in and out of the beam path while the system is in vacuum to either reflect the drive laser beam to (power meter/IR spectrometer/camera, or let the co-propagating drive laser and HH beams pass. A beamsplitter is chosen to lower the thermal stress of the neutral density filters placed in front of the camera. At optimum alignment of the capillaries, we measure a maximum capillary throughput around $60 \%$. With the maximum drive laser pulse energy of $6 \mathrm{~mJ}$ used and an estimate of $30 \%$ losses due to incoupling and scattered light at the first slit, a peak intensity of up to $1.8 \times 10^{14}$ $\mathrm{W} / \mathrm{cm}^{2}$ can be launched into the interaction length in the waveguide.

Behind the capillary, the $\mathrm{HH}$ beam and drive laser beam co-propagate over a long distance of $9 \mathrm{~m}$, to allow for the drive laser intensity to be reduced by diffraction in order to avoid ablation of the Aluminum (Al) filters which are used to block the drive laser from the XUV detection devices. For observing shot-to-shot fluctuations in the harmonic output from this setup, we record series of 100 single shots. The series of measurements are carried out for capillaries with both lengths ( $37 \mathrm{~mm}$ and $220 \mathrm{~mm}$ ) in which the gas pressure was varied from 2 mbar to 50 mbar. As in the previous setup (Chapter 3), the single shot measurements are carried out with the combination of an optical chopper and a shutter which yields a data collection rate of $3 \mathrm{~Hz}$. To measure the $\mathrm{HH}$ spectrum, a transmission grating $(10,000$ lines $/ \mathrm{mm}$ ) is moved into the $\mathrm{HH}$ beam path at $66 \mathrm{~cm}$ distance in front of the CCD camera. The measurement of the average high-harmonic spectrum is obtained by letting the camera integrate over a few hundred subsequent shots.

\subsection{Experimental Results}

For a first qualitative overview we present in Fig. 5.2 the typical single-shot CCD images of high-harmonic beam profiles, recorded with a drive pulse energy of 6.0 $\mathrm{mJ}$ for two capillaries that provide an interaction length of $37 \mathrm{~mm}$ (a) and $220 \mathrm{~mm}$ (b) while we vary the gas pressures from 2 mbar to 20 mbar.

(a)
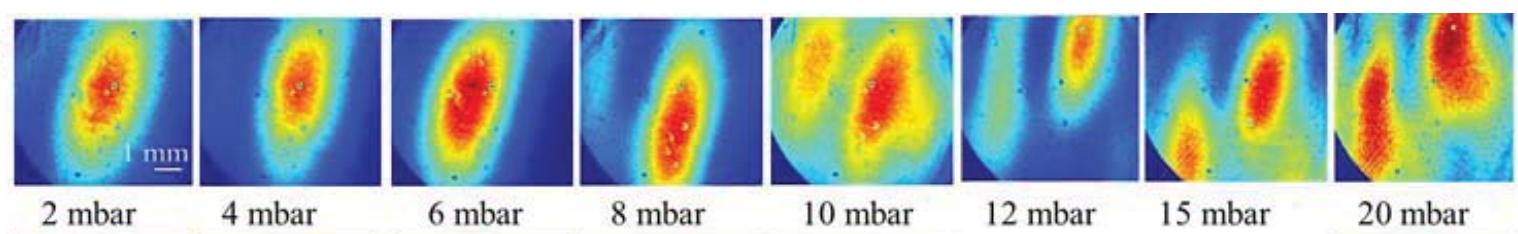

$12 \mathrm{mbar}$
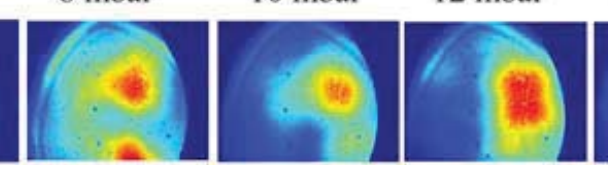

$15 \mathrm{mbar}$

20 mbar

(b)
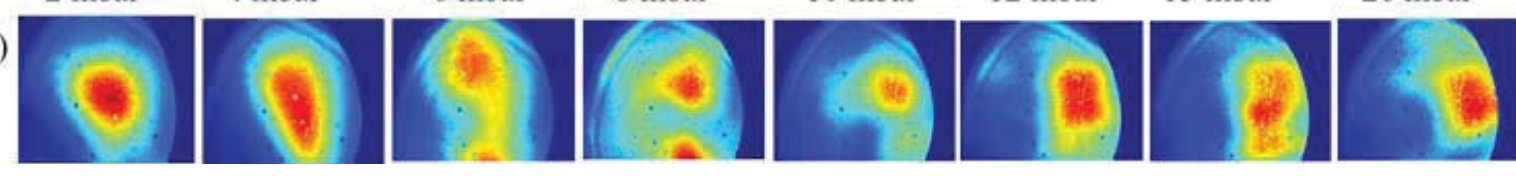

Figure 4.7: Typical single-shot CCD image of the harmonic beam as a function of Ar gas pressure at $6.0 \mathrm{~mJ}$ drive laser pulse energy and a capillary with a radius $a=254 \mathrm{~mm}$. For (a) first capillary with $L_{m}$ 
$=37 \mathrm{~mm}$ and (b) second capillary with $L_{m}=220 \mathrm{~mm}$ while we vary the gas pressure from 2 mbar to 20 mbar.

In Fig. 4.7(a), with the short capillary, we observe that in all shots the HH output possess an elliptical and tilted profile at low gas pressures from 2 mbar to 10 mbar (longer at the vertical direction). From the coincidence in direction we conclude that the elliptical beam profile is due to the slight elliptical shape of the input beam which generates an elliptical HH beam as well. As for the tilted beam profile, we note that our choice in alignment is to obtain a maximum $\mathrm{HH}$ output, rather than a $\mathrm{HH}$ beam that exhibits no tilt for certain other alignment settings.

In Fig. 4.7(b) with the long capillary, we find the harmonic beam to exhibit round, near Gaussian beam profiles at low gas pressures from 2 mbar to 4 mbar. Also, we observed that the shape of $\mathrm{HH}$ beam remains round and less sensitive to the drive laser alignment as compared to the first capillary. These observations suggest that for the second capillary with extended length, a better HH beam quality can be achieved. Since the theoretical absorption length of the HH beam is only a few $\mathrm{mm}$ in this pressure range ( 2 mbar to 20 mbar), most part of the $\mathrm{HH}$ output is coming from the end of the capillary whereas the main part of the capillary serves merely for guiding the drive laser to this generation volume near exit. Therefore, it is reasonable to assume that a higher $\mathrm{HH}$ beam quality can be achieved for a longer waveguide because this yields a more efficient spatial filtering of the drive laser into the round fundamental waveguide mode.

In Fig 4.7 (a), we observe that the beam profile of each shot becomes increasingly distorted in a complex manner which deviates noticeably from a Gaussian profile and varies from shot-to-shot when the Ar gas pressure exceeds 10 mbar. In contrast, in Fig. 4.7 (b), we see that the beam profile becomes distorted starting already from lower gas pressures at around 4 mbar. As an explanation, discussed in Chapter 3, an increase of the gas pressure leads to a larger electron density which might induce multimode propagation of the driving laser pulse when exceeding the threshold electron density given by Eq.3.2. This can result in the shape of the IR guided mode field to become significantly distorted.

For the parameters of Fig.4.7, eq. 3.2 predicts a threshold for the electron density of $n_{t h}=1.2 \times 10^{16} \mathrm{~cm}^{-3}$ and from the ADK model we obtain a gas pressure of 10 mbar to reach this threshold electron density, which agrees rather well with the onset of distorted HH beam profiles in Fig. 4.7 (a) with the short capillary. However, in Fig. 4.7 (b) for the second capillary, we see that the beam profile becomes distorted starting already from lower gas pressures at around 4 mbar where the calculated electron density is a factor of 3 lower than the threshold. So, the question to why the $\mathrm{HH}$ beam profiles become distorted at pressures lower than expected for the second capillary which has a longer interaction length is open for discussion.

We speculate that the drive laser in a wide capillary is more susceptible to ionization induced defocusing due to weaker waveguiding. Due to plasma 
defocusing, nonlinear mode coupling becomes greater [118]. Therefore, when the defocusing length, $L_{\text {def }}$ (given in Eq. 2.33) is shorter than the interaction length, $L_{m}$ the drive laser can undergo multimode propagation. To determine whether defocusing is induced by ionization in the capillaries, we calculate the $\mathrm{L}_{\text {def }}$ for all the used Ar gas pressure up to 20 mbar. At 2 mbar, the $\mathrm{L}_{\text {def }}(194 \mathrm{~mm})$ is shorter than the interaction length of the second capillary $(220 \mathrm{~mm})$, which agrees rather well with the onset of distorted HH beam profiles for the second capillary in Fig 4.7(b) at 4 mbar. Whereas at $10 \mathrm{mbar}$, the $L_{\text {def }}(36 \mathrm{~mm})$ becomes shorter than the interaction length of the first capillary $\left(L_{m}=37 \mathrm{~mm}\right)$, which coincides with the onset of distorted HH beam profiles in Fig. 4.7 (a) as well.

For comparing the measured total $\mathrm{HH}$ output with the output predicted by our model, we show in Fig. 4.8 two typical HH spectra with harmonic orders ranging from the $13^{\text {th }}$ up to the $31^{\text {st }}$ order, recorded with an input pulse energy of $6.0 \mathrm{~mJ}$ using the short capillary of (a) $L_{m}=37 \mathrm{~mm}$ at a gas pressure of $6 \mathrm{mbar}$, and using the long capillary of (b) $L_{m}=220 \mathrm{~mm}$ at a gas pressure of 4 mbar. Both spectra show the generation of 10 harmonics centered around $38 \mathrm{~nm}$ (harmonic orders of 13 through 31 ). We note that the two small peaks around the $13^{\text {th }}$ harmonic arise from second order diffraction of the $25^{\text {th }}$ and $27^{\text {th }}$ order of the grating in the detection setup.
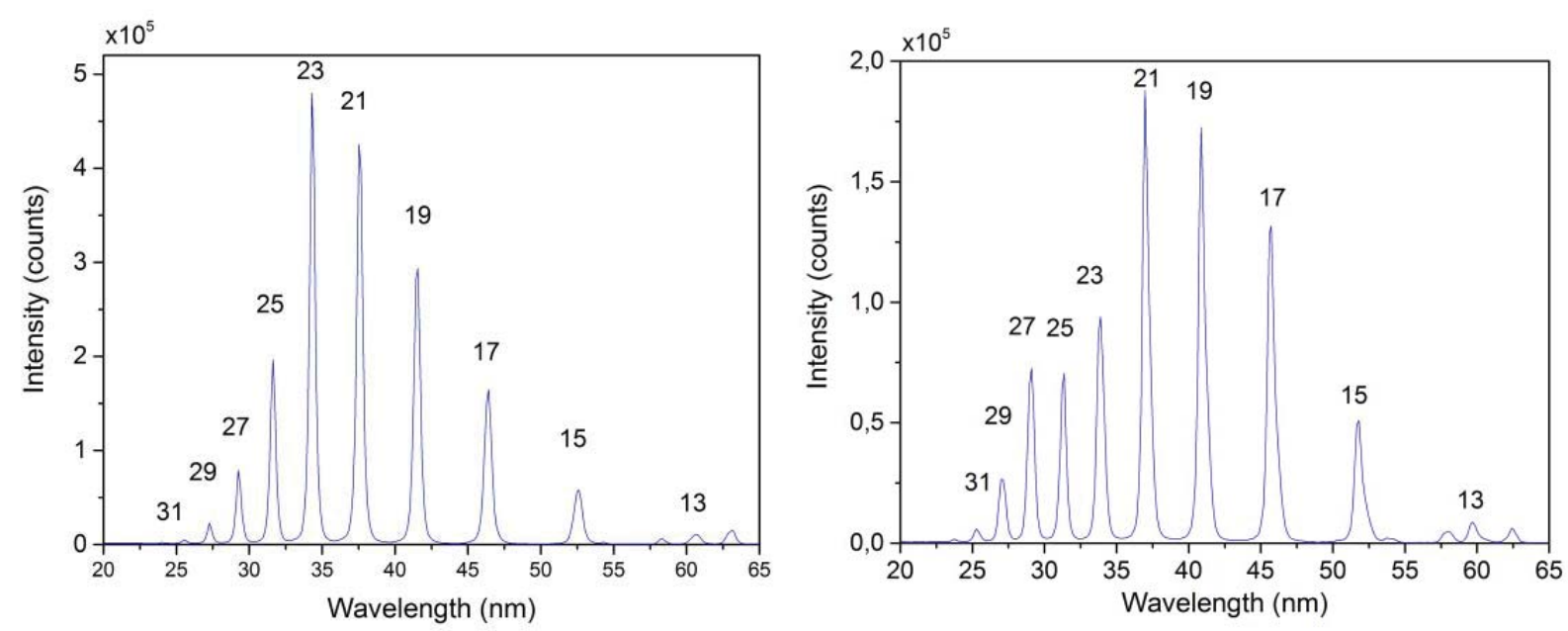

Figure 4.8: High-harmonic spectrum (integrated over 200 shots) taken with a drive laser pulse energy of $6.0 \mathrm{~mJ}$ and capillaries with a large inner radius of $a=254 \mathrm{~mm}$. (a) Spectrum obtained with the short capillary $\left(L_{m}=37 \mathrm{~mm}\right.$ at Ar gas pressure of $\left.6 \mathrm{~mJ}\right)$ and (b) spectrum obtained with the long capillary $\left(L_{m}\right.$ $=220 \mathrm{~mm}$ at Ar pressure of 4 mbar).

Combining the data from the total measured fluence (integrated spectrally over the beam profile) and the spectral distribution in Fig.4.8, we obtain an absolute value for the total pulse energy in the high-harmonic beam with the calibration procedure described previously in Sect.3.3. Figure 4.9 shows the total pulse energy averaged over 100 single shots versus pressure $(2$ mbar to 50 mbar) for the two thick 
capillaries $(a=254 \mu \mathrm{m})$ with interaction lengths of $L_{m}$ of $37 \mathrm{~mm}$ (black open circles) and $220 \mathrm{~mm}$ (square magenta). For a comparison between the HH output in a thick capillary against a thin capillary, the previously measured $\mathrm{HH}$ output pulse energies from a thin capillary ( $a=75 \mu \mathrm{m}$, see Fig.3.5(a)), are re-plotted in Fig.4.9 as a function of Ar gas pressure (40 mbar to 160 mbar) for drive energies of $0.6 \mathrm{~mJ}$ (green), $0.8 \mathrm{~mJ}$ (red), $1.0 \mathrm{~mJ}$ (blue) and $1.1 \mathrm{~mJ}$ (light blue).

As can be seen, at optimum gas pressure of 53 mbar, $\mathrm{HH}$ output from a thin capillary increases with drive energy and a highest output of $1.2 \mathrm{~nJ}$ is obtained using $1.0 \mathrm{~mJ}$ drive energy. At this gas pressure, applying even higher drive energy of 1.1 $\mathrm{mJ}$, however, leads to a reduced HH output $(1.1 \mathrm{~nJ})$. Therefore the upper limit for the drive energy that can be applied in the thin capillary for maximizing the $\mathrm{HH}$ output is $1.0 \mathrm{~mJ}$. Using a thick capillaries of inner radius, $\mathrm{a}=254 \mu \mathrm{m}$, we scale up the drive energy to $6.0 \mathrm{~mJ}$.

We measure a maximum $\mathrm{HH}$ pulse energy of $4 \mathrm{~nJ}$ at 6 mbar Ar gas pressure with the first thick capillary $\left(L_{m}=37 \mathrm{~mm}\right)$. With the second thick and long capillary $\left(L_{m}=\right.$ $220 \mathrm{~mm}$ ), we observe a maximum energy of $3.3 \mathrm{~nJ}$ at 4 mbar. These measurements show clearly that using a wider capillary is a viable method, in combination with a lower gas pressure, to scale the HH output up with the drive laser pulse energy. Here, the harmonic output is a factor of 3.3 higher using a thick capillary $(4 \mathrm{~nJ})$ as compared to using a thin capillary $(1.2 \mathrm{~nJ})$.

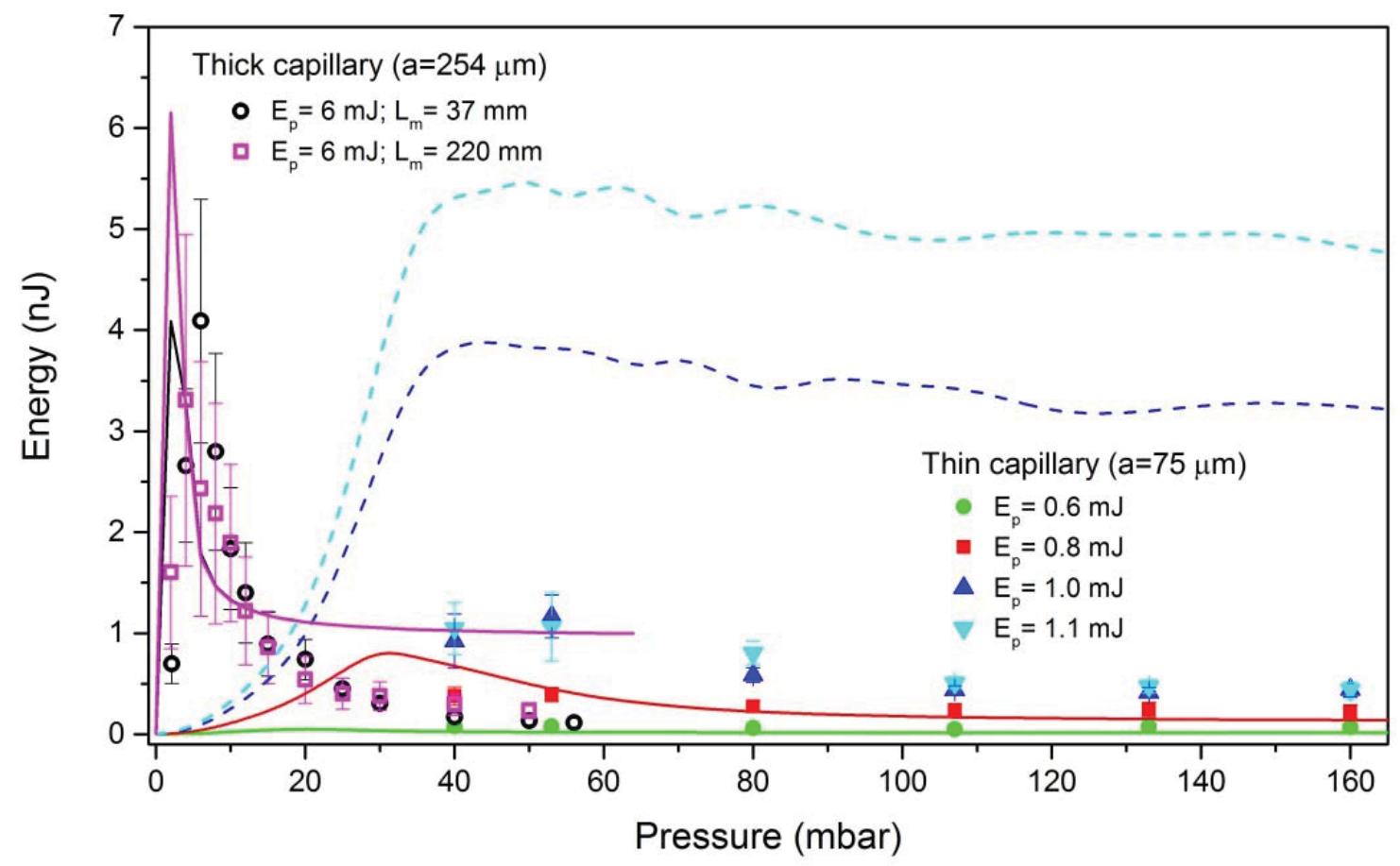

Figure 4.9: Total (spectrally integrated) pulse energy of the high-harmonic beam measured as a function of Argon pressure with thick capillary of inner radius, $a=254 \mathrm{um}$ and drive energy, $\mathrm{E}_{\mathrm{p}}=6 \mathrm{~mJ}$ for different interaction lengths, $L_{m}=37 \mathrm{~mm}$ (black open circle) and $L_{m}=220 \mathrm{~mm}$ (magenta open square). With thin capillary of inner radius, $a=75 \mu \mathrm{m}$ and $L_{m}=37 \mathrm{~mm}$ for different drive laser energies at $0.6 \mathrm{~mJ}$ (green square), $0.8 \mathrm{~mJ}$ (red circles), $1.0 \mathrm{~mJ}$ (blue triangles), and $1.1 \mathrm{~mJ}$ (purple 
diamonds). Each data point is obtained via measuring the single-shot pulse energy for 100 shots and subsequently calculating the average value and standard deviation (error bars). The theoretical curves calculated from PM model are plotted with the same color of their corresponding measured data point.

To compare the measured values with the theoretical values, we use as the input to the theoretical model the pulse energies that we have measured behind the empty capillaries, and to calculate the total output $\left(\mathrm{E}_{\mathrm{tot}}\right)$ for harmonic orders from $13^{\text {th }}$ to $31^{\text {st }}$ which corresponds to all the harmonics observed in a typical spectrum (Fig.4.8). Since $\mathrm{E}_{\text {tot }}$ represents a relative total output instead of absolute pulse energy, we decided to fit the theoretical values of $E_{\text {tot }}$ to the experimental values. The fit constant is chosen as the ratio between the maximum theoretical value $(2.4 \mathrm{~nJ}$ at 2 mbar) to the maximum measured value ( $4.0 \mathrm{~nJ}$ at $6 \mathrm{mbar}$ ) for $E_{p}=6 \mathrm{~mJ}, \eta=60 \%, a$ $=254 \mu \mathrm{m}$ and $L_{m}=37 \mathrm{~mm}$. Using the same, single fit parameter for all the calculated pulse energies, the six theoretical curves are plotted in Fig.4.9. In this plot, the color of each curve is chosen to be the same as the experimental data points with which the curve is expected to overlap.

When inspecting first the theoretical curve for $E_{p}=6 \mathrm{~mJ}$ and $L_{m}=37 \mathrm{~mm}$ (black curve), it can be seen that the model predicts a maximum output $(4.0 \mathrm{~nJ})$ at gas pressure of 2 mbar. Indeed, there is an agreement because this pressure is close to the optimum pressure, 6 mbar, obtained in the measurement. Next, inspecting the theoretical curves for $\mathrm{HH}$ output for $E_{p}=6 \mathrm{~mJ}$ and $L_{m}=220 \mathrm{~mm}$ (magenta curve) one sees that a maximum output of $6.3 \mathrm{~nJ}$ is predicted at a gas pressure of 2 mbar. We note that also this prediction agrees well with the experimental data as the maximum output is comparable (1.8 times lower) and occurs at the same pressure (2 mbar). Finally, we observe for both curves that the output level off beyond $10 \mathrm{mbar}$ while the measured values gradually decrease as well. However, there is a clear offset between theoretical and experimental values at higher pressures.

We expect that this offset is related to a defocusing of the drive laser discussed previously, where the onset of ionization induced defocusing starts from 10 mbar for $L_{m}=37 \mathrm{~mm}$ and $2 \mathrm{mbar}$ for $L_{m}=220 \mathrm{~mm}$ (see Fig.4.7). Such defocusing reduces the intensity of drive laser, which leads to a lower high-harmonic output than predicted by the model (which only account for phase-matching for the fundamental mode).

Finally, we look at the theoretical HH output predicted for the thin capillary. It can be seen that the theoretical curves for the two lower pulse energies, $E_{p}=0.6 \mathrm{~mJ}$ (solid green) and $0.8 \mathrm{~mJ}$ (solid red), match well with the experimental values. However, the comparison between the measured and calculated values for higher drive energies, at $1.0 \mathrm{~mJ}$ (blue) and $1.1 \mathrm{~mJ}$ (light blue), shows a clearly noticeable discrepancy in that the pressure of maximum generation is approximately as measured, but where the experimental output becomes much smaller than predicted beyond this pressure range (about beyond 40 ad 50 mbar). Again, we attribute the lower values in the experiment to the defocusing of the drive laser in this intensities regime. 
For more clarity, we have selectively replotted in Fig.4.10, two data sets from Figure 4.9 that were recorded with the following experimental parameters $\left(E_{p}=6\right.$ $\mathrm{mJ} ; L_{m}=37 \mathrm{~mm} ; a=75 \mu \mathrm{m}$ and $\left.E_{p}=0.8 \mathrm{~mJ} ; L_{m}=37 \mathrm{~mm} ; a=75 \mu \mathrm{m}\right)$ which produce the maximum pulse energy yet do not show any effects of ionization induced multimode propagation of the drive laser in the thick and thin capillary respectively. Their corresponding theoretical curves are plotted as well for comparison, where good overlap with the experimental data can be seen as the model assumptions hold well for these experimental parameters.

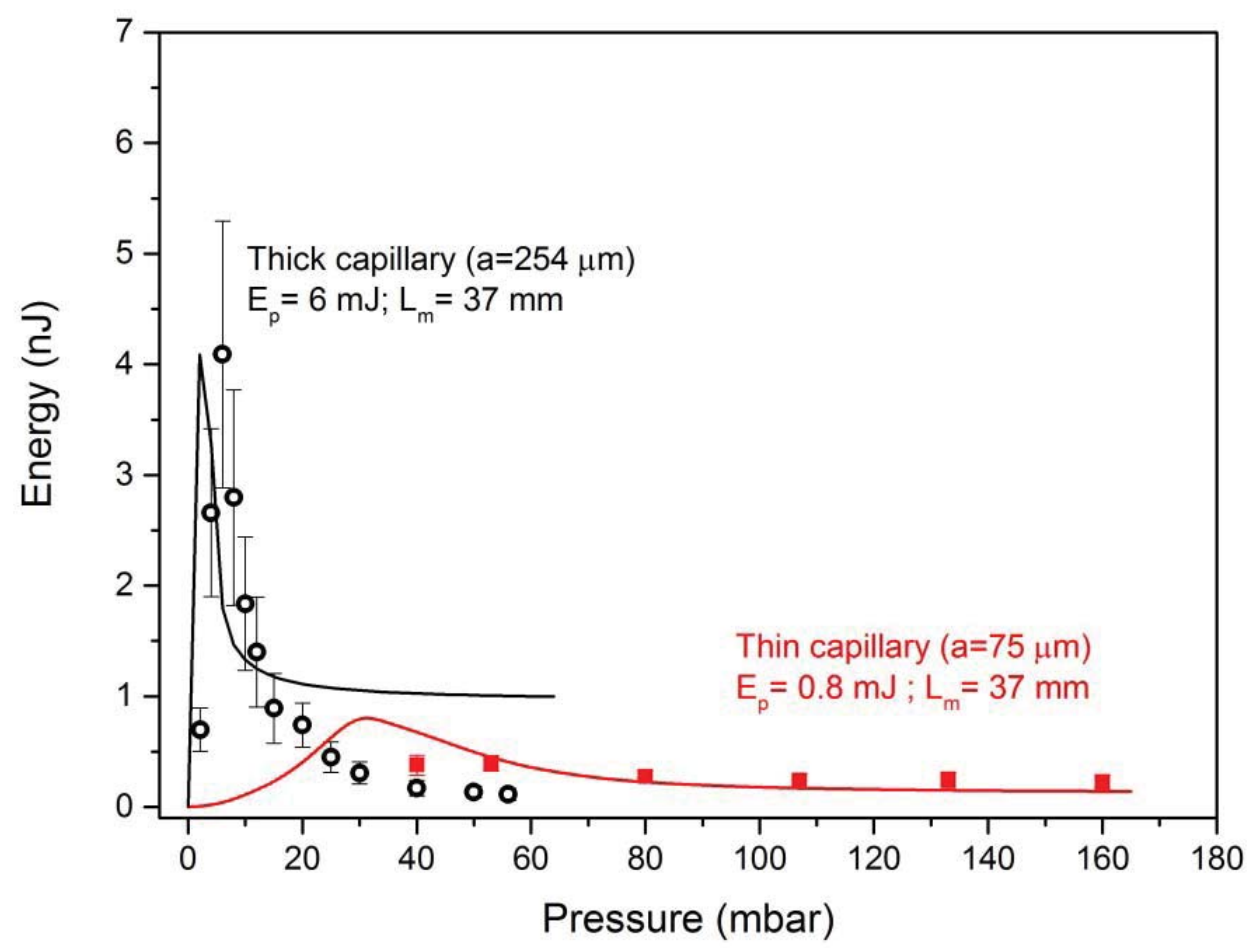

Figure 4.10: Total (spectrally integrated) pulse energy of the high-harmonic beam measured as a function of Argon pressure with thick capillary of inner radius; a $=254 \mathrm{um}$; drive energy, $E_{p}=6 \mathrm{~mJ}$ for interaction length, $L_{m}=37 \mathrm{~mm}$ (black open circle) and with thin capillary of inner radius, $a=75 \mu \mathrm{m}$; drive energy, $E_{p}=0.8 \mathrm{~mJ}$ interaction length, $L_{m}=37 \mathrm{~mm}$ (red square). Each data point is obtained via measuring the single-shot pulse energy for 100 shots and subsequently calculating the average value and standard deviation (error bars). The theoretical curves calculated from PM model are plotted with the same color of their corresponding measured data point.

In summary, we find a good qualitative agreement between the measurement and theoretical curve for certain range of low gas pressures and low drive intensities. In these pressures and drive intensities, the drive laser remains constant and propagates in single-mode throughout the interaction length. This agreement is quite noticeable because it comprises a rather wide range of experimental parameters, namely in terms of pressure, pulse energy, capillary diameter and capillary length. At higher gas pressure, drive energies and longer interaction length, however, the simple model that we have used, as was expected, loses its validity due to defocusing of the drive laser. Here, the measured HH output becomes lower than predicted. 


\subsection{Conclusions}

In this chapter, we have investigated theoretically and experimentally the enhancement of the high-harmonic output energy when we scale up the capillary radius together with the drive energy. We developed a model which calculates the build-up of high-harmonic energy in an Ar-filled capillary, taking into account spatiotemporal phase matching separately at all relevant harmonic orders and also the spectrally varying reabsorption of generated harmonic radiation. With the model, we calculate the relative yield of high-harmonic energy when we scale up the radius of the capillary with the drive energy. Experimentally, we generate high-harmonics using two Ar-filled capillaries of large inner radius $(254 \mu \mathrm{m})$ together with increased pulse energy of up to $6 \mathrm{~mJ}$. To our knowledge, this is the first experimental demonstration of high-harmonic generation using waveguides with such wide an inner radius. Using this capillary waveguides, we have achieved a factor of 3.3 enhancement in the output energy $(4 \mathrm{~nJ})$ compared to measurements that use standard capillary with small inner radius of $75 \mu \mathrm{m}(1.2 \mathrm{~nJ})$. Lastly, we compared the experimental $\mathrm{HH}$ output energies values with the theoretical predicted values over a wide range of gas pressure. We found the theory to agree well with the experimental values for a certain range of lower gas pressure in thick capillaries and lower intensities in thin capillary where the degree of ionization is kept low. Because the agreement comprises an extended range of various, central parameters for $\mathrm{HHG}$, i.e., the pressure, drive laser pulse energy, capillary diameter and capillary length, the devised model, despite its simplicity and limitations appears to be a good guide for upscaling waveguided HHG with the drive laser pulse energy. 


\section{5}

\section{Spectral control of high-harmonic generation in a wide-diameter capillary}

\subsection{Introduction}

High-harmonic generation (HHG) is a nonlinear optical process that provides coherent radiation in the form of ultra-short pulses, covering a broad spectrum including the extreme ultraviolet (XUV). In recent years, HHG has found numerous applications such as in imaging at the nanoscale [44], XUV interferometry [47] or spectroscopy [45, 46] while future applications are envisioned, e.g, the spectral control of free-electron lasers via injection seeding [60]. All these applications would clearly benefit if elevated drive laser pulse energies are applied for increasing the high-harmonic $(\mathrm{HH})$ output [53]. However, when using higher drive pulse energies it remains essential to maintain control over the spatial and spectral properties of the generated radiation. For spatial control of HHG along increased interaction length, capillaries seem particularly promising, due to tight waveguiding of the drive laser pulses $[86,103]$. Additionally in thin capillaries, spectral control in terms of wavelength tuning of the various harmonic orders can be gained via pulse shaping of the drive laser [48]. However, in these standard capillaries, where the inner diameter is small (typically $150 \mu \mathrm{m}$ diameter), HHG suffers from several fundamental limitations. First, the output pulse energies are limited by ionizationinduced phase mismatching already at relatively low drive laser pulse energies, typically beyond a few hundred $\mu \mathrm{J}$. Second, the drive laser propagation becomes subject to complicated nonlinear propagation effects. These increase the output under certain circumstance, through temporal self-compression [57], but otherwise reduce the degree of control. The latter shows up as multimodal drive laser propagation and nonlinear mode coupling caused by ionization-induced defocusing [57], and can lead to significant spatial shot-to-shot fluctuations [119].

A promising way to overcome such limitations and still maintain spatial and spectral control would be HHG in a capillary with significantly increased cross section, thereby limiting the intensity such that mJ-levels of drive laser pulse energies can be applied without inducing undesired nonlinear pulse propagation dynamics. However, such experiments have not been done before and simple scaling arguments are not straightforward. For instance, in a wide capillary where the 
waveguiding induced dispersion is weaker, phase matching occurs at lower gas pressure. This might impose limitations on the spectral control via drive laser pulse shaping because such technique involves a critical timing and sizing of a blue shift of the drive laser induced by ionization [48]. It is therefore important to perform an experimental investigation of the spectral control of HHG in wide-diameter capillaries for identifying the effectiveness of drive laser pulse shaping.

Here we present the first investigation of spectral control of HHG through drive laser pulse shaping with a wide-diameter capillary. The results show that, in spite of rather different operational parameters in such capillaries, compared to thin standard capillaries, pulse shaping induced spectral control remains effective and attractive for applications.

\subsection{Experimental details}

The overall setup used for the experiments is schematically depicted in Fig. 5.1. To drive the HHG, we employ a pulsed Ti:Sapphire infrared (IR) laser with $795 \mathrm{~nm}$ center wavelength at a repetition rate of $1 \mathrm{kHz}$ (Legend Elite Duo HP USP, Coherent Inc.). The shortest duration of the infrared (IR) laser pulses is $40 \mathrm{fs}$ as measured with frequency resolved optical grating (Grenouille) [96]. The maximum available pulse energy in the experiments was up to $6.9 \mathrm{~mJ}$. The pulses are close to the Fourier limit (time-bandwidth product of 0.5 ) and the beam parameter, $\mathrm{M}^{2}$, is close to unity $\left(M_{x}^{2}=1.1 ; M_{y}^{2}=1.3\right)$.

Most of the experimental setup has been described in the previous chapter, but here we recall the setup for convenience and for a more detailed description of how drive laser pulse shaping is implemented. A $67 \mathrm{~mm}$ long capillary-waveguide (interaction length of $L_{m}=37 \mathrm{~mm}$ ) with a $508 \mu \mathrm{m}$ wide inner diameter is mounted in a vacuum chamber and is filled with Ar gas at various pressures. For compensation of residual astigmatism and mode matching the drive laser beam to the lowest order waveguiding capillary mode, we use a weakly focusing mirror $(2.5 \mathrm{~m}$ focal length) under $14^{\circ}$ angle of incidence. At optimum alignment of the capillary, we measure a maximum drive laser throughput of around $60 \%$. With the drive laser pulse energy of $6.9 \mathrm{~mJ}$ used and an estimate of $30 \%$ losses due to incoupling and scattered light at the first slit, a peak intensity of up to $2.1 \times 10^{14} \mathrm{~W} / \mathrm{cm}^{2}$ can be generated in the waveguide.

As was described in Section 4.4, behind the capillary, we let the high-harmonic and drive laser beams co-propagate over a large distance $(9 \mathrm{~m})$, to reduce the drive laser intensity by diffraction. The drive laser beam is then blocked by a set of two Aluminum (Al) filters placed in series, each being $200 \mathrm{~nm}$ thick. For spectral analysis the XUV radiation is sent to a transmission spectrometer equipped with home-fabricated high-line-density gratings [98]. The Al filters also act as a bandpass for XUV radiation, leaving a spectral transmission window from $16 \mathrm{~nm}$ to $80 \mathrm{~nm}$. 
Behind the Al filters the radiation is detected with an XUV CCD camera (Andor DO240). The signal obtained here is carefully calibrated, via the spectrally dependent camera sensitivity and transmission of Al filters, to allow for a determination of absolute pulse energies.

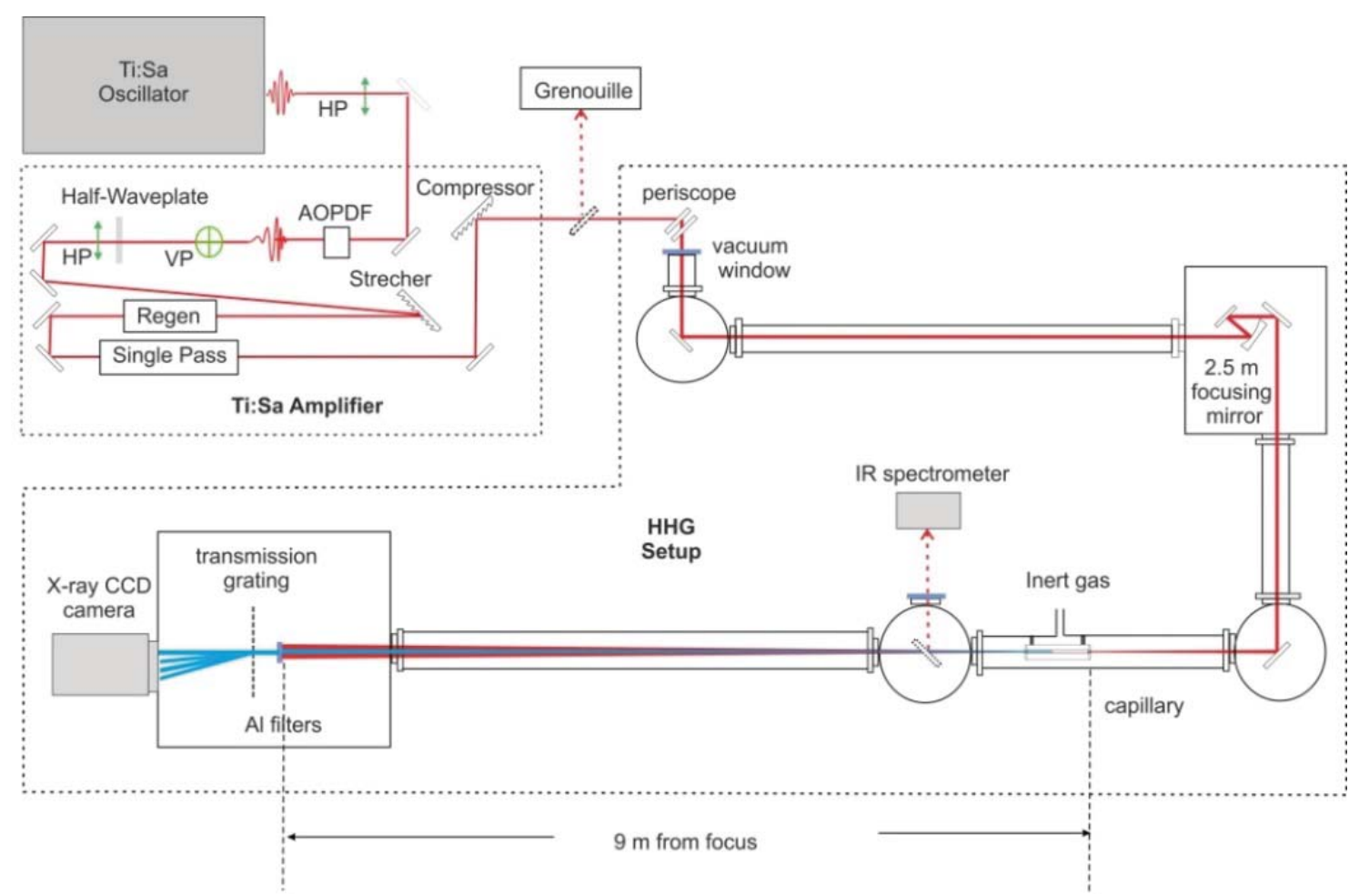

Figure 5.1: Schematic of the experimental setup for spectral tuning of high-harmonic generation (HHG) via drive laser pulse shaping in a wide capillary. The drive laser system incorporates an acousto-optic programmable dispersive filter (AOPDF) for phase shaping of the drive laser pulses (HP = horizontal polarization; $\mathrm{VP}=$ vertical polarization). The shaped pulses are sent to Grenouille measurements of the pulse duration and into an Ar-filled (508 $\mu \mathrm{m}$ wide) capillary for high-harmonic generation. Spectral tuning of HHG is observed with a transmission XUV spectrometer.

During the course of the experiments we investigate spectral control through two methods, through a variation of drive laser chirp and through a variation of the gas pressure. Drive laser pulse shaping is performed with an acousto-optic programmable dispersive filter (AOPDF, DAZZLER HR-800/T1, Fastlite) [120]. As shown in Fig.5.1, the programmable filter is positioned behind the laser oscillator in order to prevent optical damage. The polarization of the shaped pulse leaving the programmable filter, which is rotated by 90 degree, is rotated back with a half-wave plate. The pulse is then stretched and injected into the regenerative amplifier followed by single pass amplification. After amplification, the pulse is sent to the compressor. For a calibration of the various phase settings applied to the dispersive filter, we measure the according pulse duration of the drive laser after the compressor using a Grenouille [96]. The experimental error of the pulse measurement using the Grenouille is estimated to be $\pm 2.5 \mathrm{fs}$. Although a large 
variety of different pulse shapes can be provided with the programmable filter via amplitude and phase shaping, here we restrict ourselves to phase shaping in order to avoid loss of pulse energy. For concentrating on the most basic effects only, we restrict ourselves to shaping with second-order-parabolic phase spectra which corresponds to generating drive laser pulses with various degrees of linear chirp.

To enable a comparison of the wavelength shift of the high-harmonic output with a possible wavelength shift of the drive laser acquired during propagation through the gas-filled capillary, we measure the transmitted drive laser behind the gas-filled capillary for varying values of drive laser chirp and gas pressure. For this purpose, a flip mirror is inserted at a distance of $2 \mathrm{~m}$ behind the capillary, which sends the drive laser beam into an IR spectrometer (Ocean Optics 2000).

\subsection{Experimental results}

To demonstrate that the high-harmonic output can be increased noticeably by using higher drive laser pulse energies in combination with a wider diameter capillary, we measure the harmonic output pulse energy obtained with the $508 \mu \mathrm{m}$ diameter capillary and compare it with our previous measurement (see Chapter 3 and [119]) that use a thin $(150 \mu \mathrm{m}$ diameter) standard capillary. Figure 5.2 shows the total (spectrally integrated) pulse energy in the harmonic beam averaged over 100 single shots versus pressure for both capillaries. It can be seen that the highest output of 4.1 $\mathrm{nJ}$ at $6 \mathrm{mbar}$ is obtained from the wide capillary as compared to $1.2 \mathrm{~nJ}$ at $53 \mathrm{mbar}$ from the standard capillary. This corresponds to a factor of 3.3 increase in the harmonic output from the wide capillary which is why we are interested to investigate also the degree of spectral control that can be obtained at such elevated output.

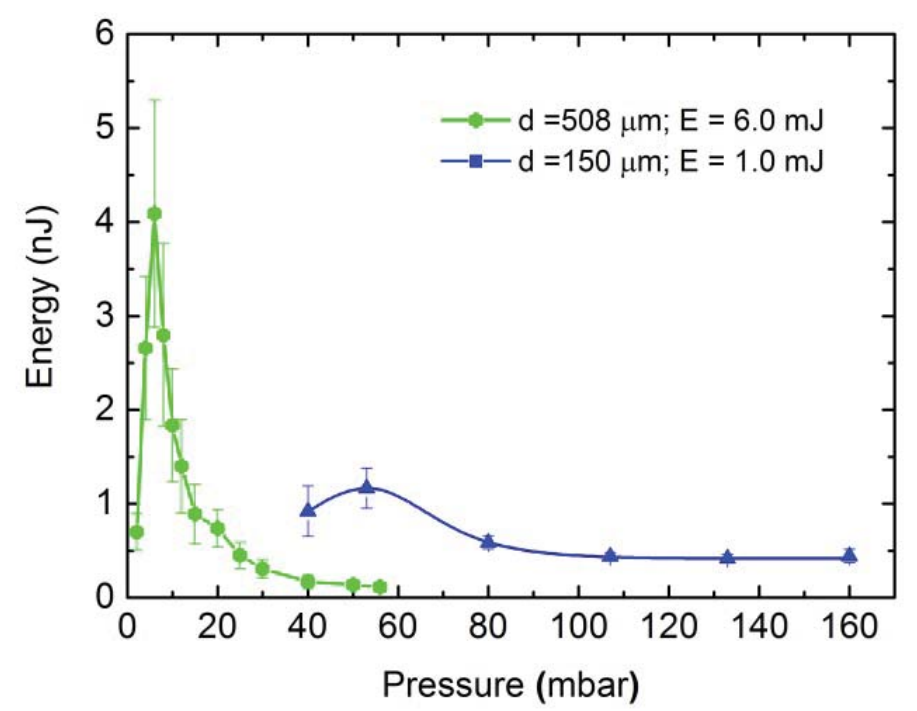

Figure 5.2: Total (spectrally integrated) pulse energy of the high-harmonic beam measured as a function of $\mathrm{Ar}$ gas pressure for two different capillary diameters, for $d=150 \mu \mathrm{m}$ (triangular symbols, 
standard capillary) with a drive energy of $1.0 \mathrm{~mJ}$ and for $d=508 \mu \mathrm{m}$ (square symbols, wide capillary) with $E=6.0 \mathrm{~mJ}$. The shown pulse energies are obtained by measuring the individual pulse energies of 100 single shots and taking the average. The error bars show the pulse-to-pulse root mean square deviation.

Now turning to the spectral investigation, in Fig. 5.3(a), we show a series of high-harmonic spectra recorded with the wide capillary for various different Ar gas pressures, when pumped with the shortest (40 fs) drive laser pulse, i.e., using drive laser pulses with zero chirp. The spectra are obtained by integrating the HH output over 20 pulses. In the spectra, the harmonic orders, $q$, between 17 and 29 can be seen as spectral peaks. Each spectrum is normalized to the highest peak, while the different spectra are offset with regard to each other by the same vertical distance for more clarity. As the pressure increases from 2 mbar to $10 \mathrm{mbar}$, we observe that the order of the strongest harmonic increases from the $q=17$ to 27 . This observation is consistent with what was reported in Ref.[121] and can be understood as due to the pressure dependence of phase matching and reabsorption of harmonics. A second effect can be identified in the spectra, which is a wavelength shift (tuning) of all harmonic orders with the pressure.

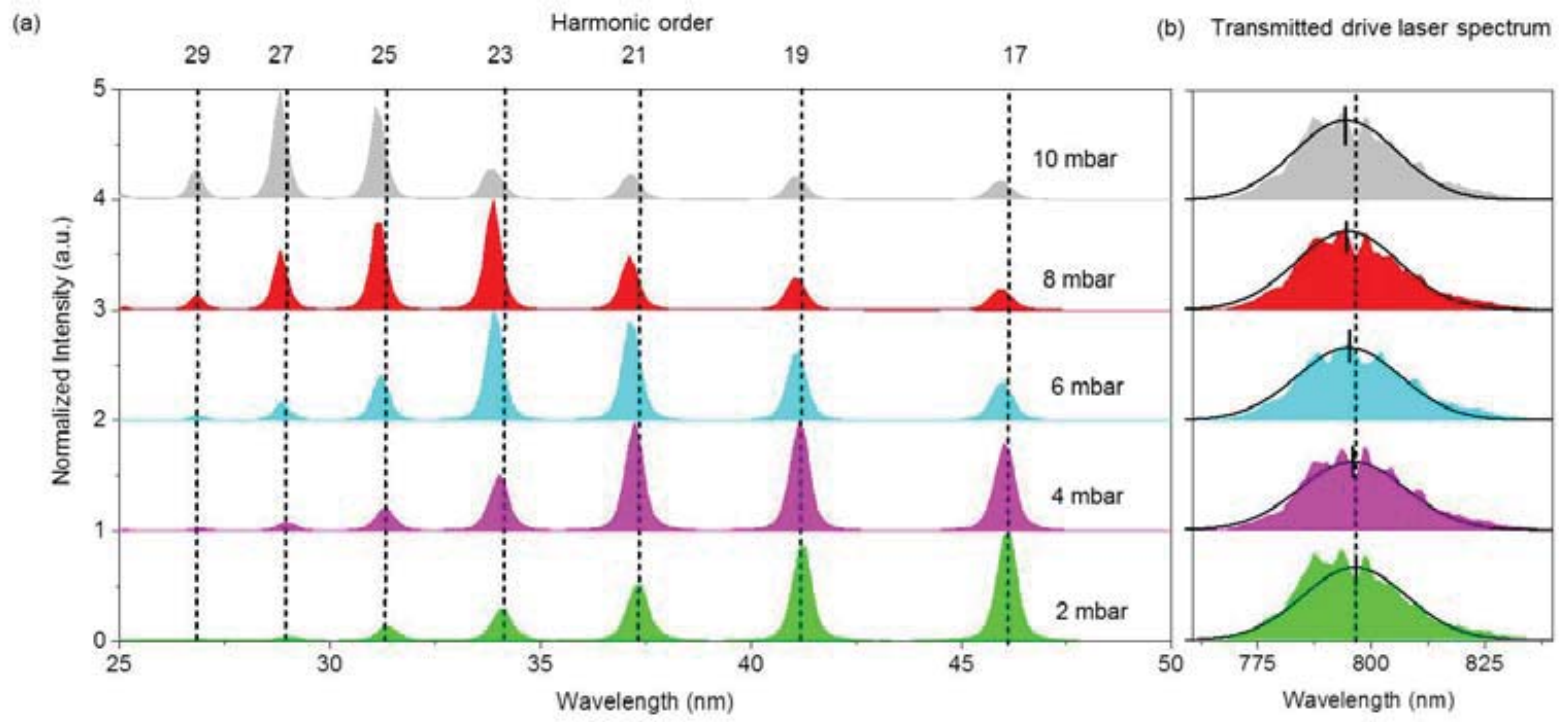

Figure 5.3: (a) High-harmonic and (b) drive laser spectra measured behind the capillary filled with different Ar gas pressure, for a drive laser energy of $\mathrm{E}=6.9 \mathrm{~mJ}$ and with zero chirp setting of the programmable filter. The dashed lines indicate the center wavelengths of the harmonic orders and of the transmitted drive laser recorded at the lowest Ar gas pressure of 2 mbar whereas the short solid lines in (b) show the measured center wavelength.

As a reference for later evaluation, we have indicated the original spectral positions of the harmonic orders (Gaussian least-square fit of the harmonic peaks) measured at the lowest pressure, 2 mbar) as vertical dashed lines. For a discussion of the physical processes responsible for the high-harmonic blue-shift, we have plotted in Fig5.3(b) the corresponding spectra of the drive laser radiation as recorded after 
interaction with the $\mathrm{Ar}$ gas (transmitted through the capillary). The center wavelength of the drive laser spectra, as indicated by short vertical line, is obtained again with a Gaussian least-square fit. The vertical dashed line indicates the center wavelength at the lowest pressure of 2 mbar. It can be seen that, similar to the harmonic orders, the drive laser central wavelength shows an increasing blue-shift as the gas pressure increases from 2 to 10 mbar.

In Fig.5.4(a), we show a series of high-harmonic spectra recorded at a fixed gas pressure of 6 mbar with various different drive laser pulse durations (obtained with according chirp settings at the programmable filter). The corresponding drive laser spectra measured behind the capillary are given in Fig.5.4 (b) where the vertical dashed line indicates the center wavelength of the drive laser pulse measured behind the empty capillary $\left(\lambda_{L}\right)$, i.e., without having any interaction with Ar gas. For better clarity and for the discussion that follows, we draw vertical dashed lines in Fig. 5.4 (a) that indicate the estimated center wavelengths of the harmonic orders in the absence of ionization-induced blue shift. We define the estimated wavelengths (dashed lines)

$$
\lambda_{q}+\frac{\lambda_{L}-\lambda_{L}(6 \mathrm{mbar})}{q},
$$

where $\lambda_{q}$ is the center wavelength of the harmonic $q^{\text {th }}$ and $\lambda_{L}(6$ mbar $)$ is the center wavelength of the drive laser after interaction with $6 \mathrm{mbar}$ Ar gas. Both $\lambda_{q}$ and $\lambda_{L}(6$ mbar) are recorded at zero chirp setting (40 fs). The sign given with the pulse duration (FWHM in fs) corresponds to the sign of the applied laser chirp. During the measurements, the driving pulse energy is kept constant and hence its intensity becomes lower as the pulse duration becomes longer.

In Fig.5.4(a) we observe harmonics ranging from $q=17$ to 29 and the strongest harmonic is the $23^{\text {rd }}$ for most cases. This indicates that, unlike in the measurements for Fig.5.3, the phase matching conditions are approximately unchanged during the pulse shaping experiments. We observe a second effect in the spectra, which is a wavelength shift (tuning) of all harmonic order with the pulse duration. Here, the blue-shift of the harmonic peaks is hardly visible when positive chirp ( +95 and +57 $\mathrm{fs}$ ) is applied to the drive pulses. At zero chirp (40 fs), the blue-shift become clearly visible. However, for negative chirp, at -48 fs and $-68 \mathrm{fs}$, the harmonic peaks show an even larger blue-shift than that for zero chirp (40 fs) while in Fig.5.4 (b) (drive laser spectrum), it can be seen that highest blue-shift occurs at minimum (zero) chirp (40 fs). Lastly, at $-98 \mathrm{fs}$, the harmonic peaks show a large red-shift while the drive laser is clearly shown to be blueshifted in Fig. 5.4(b). In the following we will discuss the implication of these observations. 
(a)

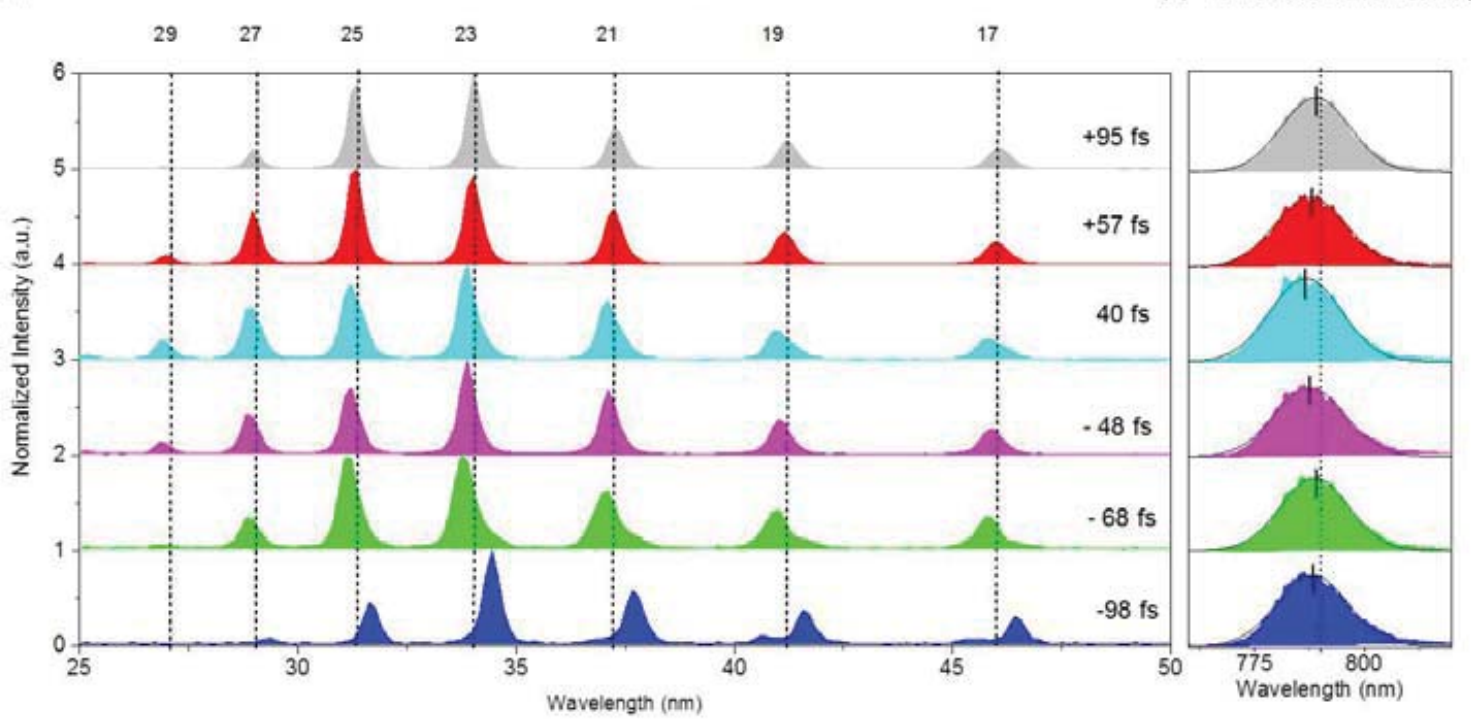

Figure 5.4: (a) High-harmonic and (b) drive laser spectra measured behind the capillary filled with 6 mbar of Ar with a drive laser energy of $E=6.9 \mathrm{~mJ}$ and with various different values of chirps applied. The sign of the pulse duration corresponds to the sign of the applied chirp. The vertical dashed line in (b) shows the center wavelength of the drive laser transmitted through an empty capillary (no Ar gas), whereas the short solid lines show the measured center wavelengths in the presence of gas. The vertical dashed lines in (a) show the center wavelengths of the harmonic orders as expected for the absence of any drive laser wavelength shift as calculated with Eq.5.1.

From Fig.5.3 and Fig.5.4, we clearly observe a shift of the high-harmonic output wavelengths as a function of pressure and drive laser chirp. When looking at the observations of others [48, 122] although based on different experimental parameters (drive laser peak intensity of $I_{p}=1.2 \times 10^{14} \mathrm{~W} / \mathrm{cm}^{2}$ and an Ar gas pressure of $P=80 \mathrm{mbar}$ in Ref.[48], or $I_{p}=9 \times 10^{15} \mathrm{~W} / \mathrm{cm}^{2}$ and $P=40 \mathrm{mbar}$ in Ref.[122]), the blue-shift may result from three mechanisms. One of the mechanisms is an ionization induced blue shift of the drive laser. The ultrafast rising of the freeelectron density during the drive laser pulse causes a rapid decrease of the refractive index, which generates a temporally increasing instantaneous frequency, $\omega_{\mathrm{L}}(\mathrm{t})$ [48]. In this case the frequencies of the high-harmonic orders, $\omega_{\mathrm{hh}}=q \cdot \omega_{\mathrm{L}}(\mathrm{t})$, become blueshifted as well. In this sense, the high-harmonic output adiabatically follows the frequency shift of the drive laser. If we denote the blue-shift of the drive laser wavelength as $\delta \lambda$, the corresponding blue-shift of the $q^{\text {th }}$ harmonic order is expected to amount to a value of $\delta \lambda / q$.

A second mechanism called non-adiabatic shift [122] occurs due to nonadiabatic response of the drive medium to the rapidly increasing field strength of an ultrashort drive laser pulse. This mechanism is originates from the intensitydependent phase of the generated high-harmonic field where the harmonic undergoes blue-shift during the leading edge of the drive laser pulse and a red-shift at the trailing edge. When the drive laser peak intensity is higher than the so-called 
saturation intensity, high-harmonics are generated only in the leading edge. Coarsely, the saturation intensity corresponds to an intensity when the so-called critical fractional ionization, $\eta_{\mathrm{cr}}$, is reached beyond which high-harmonics can no longer be phase matched [54]. In this case the harmonics will be blueshifted and the degree of blue-shift is dependent on the harmonic order. For short electron trajectories which are mainly contributing to HHG in a capillary [112], the degree of blue-shift increases with harmonic order [122].

The third mechanism is related to the degree and sign of the linear chirp of the drive laser pulse. For instance, if the driving pulse is positively chirped, the instantaneous frequency grows with time, i.e., the carrier frequency at the leading edge is lower than at the trailing edge. In this case, as discussed before, when the peak intensity is higher than the saturation intensity and high-harmonics are generated only at the leading edge of the pulse, the high-harmonic generation is driven by the longer wavelength part of the drive laser spectrum and the generated harmonics experience a red-shift. Similarly, with a negatively chirped drive laser pulse we expect a blue-shift of the generated harmonics.

To investigate which of the discussed mechanisms may be responsible for the observed blue-shift in our measurements, we carry out a qualitative analysis via the relative (or fractional) wavelength shifts that the various different harmonics and the drive laser undergo which we define as $\delta \lambda_{q} / \lambda_{q}$ where $\lambda_{q}$ is the center wavelength of the $q^{\text {th }}$ harmonic and $\delta \lambda_{q}$ is the shift of $\lambda_{q}$. This definition is chosen for simple comparison with the case that HHG adiabatically follows the drive laser frequency, $\omega_{\mathrm{HH}}=q \omega_{\mathrm{L}}$. If the drive laser frequency is shifted by $\delta \omega_{\mathrm{L}}$, one obtains frequency shifted harmonic, i.e., $\omega_{\mathrm{HH}}+\delta \omega_{\mathrm{HH}}=q\left(\omega_{\mathrm{L}}+\delta \omega_{\mathrm{L}}\right)$. In terms of wavelength, $\lambda=2 \pi \mathrm{c} / \omega$, this reveals that $\delta \lambda_{q} / \lambda_{q}=\left|\delta \omega_{\mathrm{L}} / \omega_{\mathrm{L}}\right|$, independent of the considered harmonic order.

Figure 5.5(a) shows the measured relative shift of three selected harmonic orders, i.e., the value of $\delta \lambda_{q} / \lambda_{q}$, plotted as a function of gas pressure as extracted from Fig.5.3 where $\delta \lambda_{q}$ is the shift relative to the harmonic center wavelength at the lowest pressure (indicated as dashed line in Fig. 5.3). For the plot we selected the most representative orders, i.e., the lowest, the central and the highest order that were observed ( $q=17,21$, and 25) and we also plot the measured shift of the drive laser for which $q=1$. A weakly curved, almost linear increase of the blue-shift with pressure can be seen for the drive laser as is indicated by the dashed line (linear least-square fit) at $q=1$. This observation can be explained by a linearly increasing pressure leading to a linearly increasing electron density which should be approximately proportional to the ionization induced blue shift of the drive laser. As was described before, if the wavelength shift of the high-harmonics were just caused by conversion of the frequency-shifted drive laser, then the relative wavelength shift, $\delta \lambda_{q} / \lambda_{q}$ would be the same for all orders, i.e., lie along the dashed line as well. When looking at Fig.5.5(a), however, it can be seen that the relative wavelength 
shift for $q=17,21$, and 25 is larger than for $q=1$, and that the relative shift actually increases with $q$. This implies that other mechanisms are contributing to the overall harmonic blue- shift.

In order to check for the possibility that a non-adiabatic blue shift may have occurred, we use the Ammosov-Delone-Krainov (ADK) model [67] for calculating the fractional ionization, $\eta$, at peak intensity. The calculation yields a value of $\eta \approx$ $7 \%$ for the peak intensity applied in our experiments $\left(I_{p}=2.1 \times 10^{14} \mathrm{~W} / \mathrm{cm}^{2}\right)$. The maximum fractional ionization that still allows phase matching, also called the critical fractional ionization, was calculated to be $\eta_{\mathrm{cr}}=5 \%$ using the following expression [123]

$$
\eta_{c r}=\left(1+\frac{\lambda_{L}^{2} r_{e} N_{a t m}}{2 \pi \Delta n}\right)^{-1} .
$$

Here, $\mathrm{r}_{\mathrm{e}}$ is the classical electron radius, $N_{a t m}$ is the atomic number density at ambient pressure, $\Delta n$ is the difference in the indices of refraction of the gas at the fundamental and high-harmonic wavelengths. As the calculated $\eta_{c r}$ is less than the expected ionization level for the peak intensity of the driving pulse, i.e., the peak intensity is higher than the saturation intensity, the condition for observing a nonadiabatic blue shift is fulfilled [122]. This is consistent with our observation that the relative shift increases with the order, which matches with earlier results from [122], where a non-adiabatic blue-shift is reported as well. It can be concluded that the total observed harmonic blue-shift obtained is due to both ionization and nonadiabatic effects.

As for the third mechanism discussed before, a positive (negative) linear chirp of the drive laser pulse would lead to a red-shift (blue-shift) in the harmonics, given that the harmonics are generated only at the leading edge of the drive laser. If this mechanism were significant, it would allow for spectral control of the highharmonics via the drive laser chirp. To investigate whether this is the case, we plot the relative wavelength shift in Fig.5.5(b), $\delta \lambda_{q} / \lambda_{q}$, for the same harmonic orders ( $q=$ $17,21,25)$ and the drive laser $(q=1)$ but now as a function of the drive laser chirp. These data are extracted from Fig.5.4 where $\delta \lambda_{q}$ is the shift relative to the estimated center wavelength of the harmonic order in the absence of ionization-induced blueshift (indicated as dashed lines in Fig. 5.4). The shortest pulse duration of 40 fs corresponds to the zero chirp setting at the programmable dispersive filter. Settings with positive or negative chirp are indicated as an increased pulse duration with a positive or negative sign, respectively. As the pulse energy is constant, we expect that applying a chirp whether positive or negative, would let the driving laser pulse experience less ionization and less blue-shift due to the lower peak intensity. Also the blue-shift from a non-adiabatic response reduces. Therefore, if only these two mechanisms are contributing to the harmonic shift, we expect that the blue-shift of the harmonics is independent of the sign of the chirp. 
From Fig. 5.5(b), we indeed see that the blue-shift of the harmonics decreases relative to the zero chirp setting ( $40 \mathrm{fs}$ ) when the pulse duration is increased from +57 fs to +95 fs. However, for an increasingly negative chirped drive laser, from -48 fs to $-68 \mathrm{fs}$, the blue shift of harmonic becomes stronger as is expressed by the tilted straight lines (linear least-square fit) that we have fit to the data points (except for 98 fs, see discussion below). These observations match well with experiments described in Ref. [124] where the same Ar gas pressure (5 Torr) was employed. We conclude that in our case the harmonic blue-shift at negative chirp can be understood as being generated at the leading edge, and thereby a shorter wavelength is generated here as compared to positive chirp.

As we apply an even higher negative chirp to the drive laser (data points at -98 fs), we observe that the harmonics are no longer blue shifted. Instead, the harmonics show a significant amount of red-shift compared to a pulse with similar duration but with the opposite chirp ( $+95 \mathrm{fs}$ ). Also, a broadened bandwidth is observed only for 98 fs (shown in Fig. 5.4). As a possible explanation, it has been reported that for drive pulses with negative chirp, a larger chirp and bandwidth may be induced in the harmonics than in the case of transform-limited pulse [75]. This can be understood as the combined result of the chirp of the drive laser and the intrinsic negative chirp of the harmonics, the latter caused by the increasing intensity in the leading edge. This may explain why at -98 fs the harmonic spectrum shows broadened peaks as well as a significant red-shift, as compared to other pulse durations. We conclude that the total observed harmonic blue-shift is resulting from an interplay between an ionization-induced shift of the drive laser wavelength, a non-adiabatic shift and the setting of the drive laser chirp.
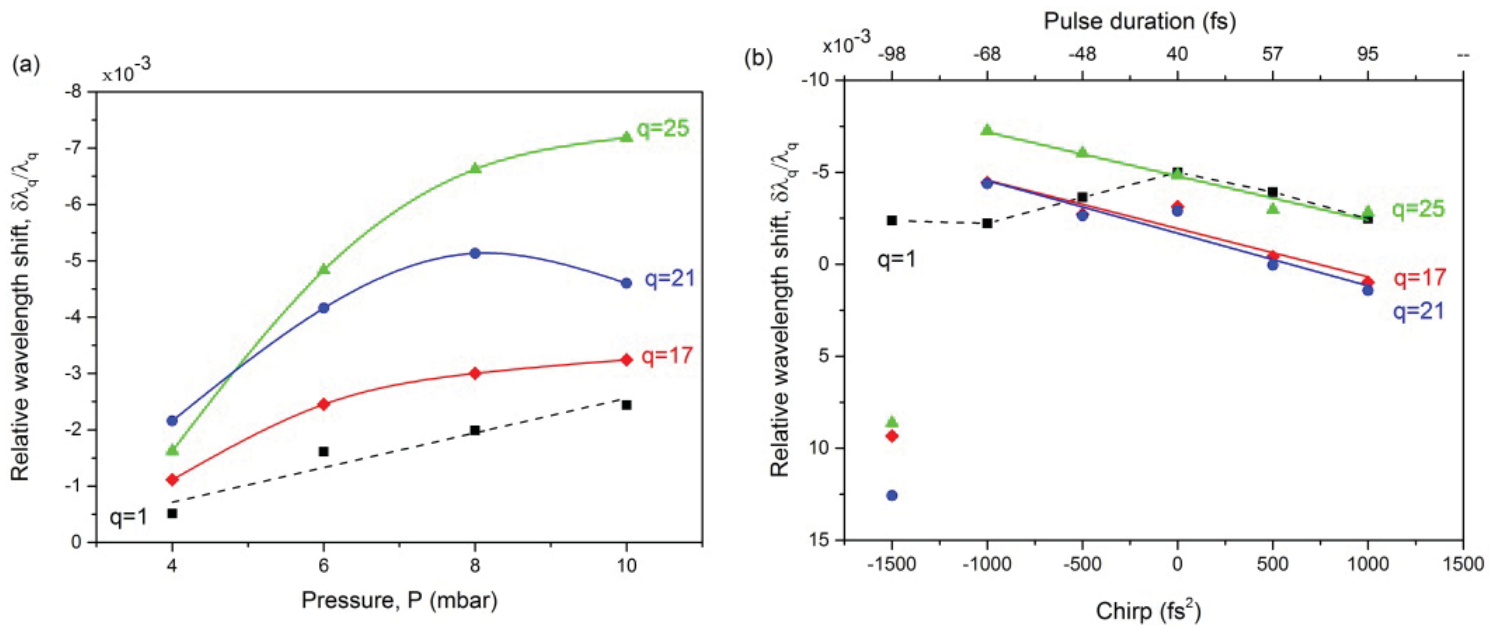

Figure 5.5: Measured relative wavelength shift of the $17^{\text {th }}, 21^{\text {st }}$ and $25^{\text {th }}$ harmonic orders as a function of (a) gas pressure and (b) as function of a linear chirp of the drive laser pulses (expressed as various pulse durations). The sign given with the pulse duration corresponds to the sign of the applied drive laser chirp, and the shortest pulse duration of $40 \mathrm{fs}$ is achieved with a zero chirp setting $\left(0 \mathrm{fs}^{2}\right)$. Negative values of the relative wavelength shift on the vertical axes represent a blue-shift. The dashed line indicates the relative blue shift of the drive laser. 
To compare the harmonic wavelength shift that we obtained via chirping of the drive laser pulses to that observed with a standard capillary of small diameter $(150 \mu \mathrm{m})$, we plotted the results reported by others [48] as the dashed dotted line in Fig.5.6. We note that in Ref. [48], $\delta \lambda_{q}$ is given as the shift relative to the harmonic center wavelength with zero chirp setting at a gas pressure of $80 \mathrm{mbar}$. To enable a direct comparison with our results, we plot our measurements for $\delta \lambda_{q} / \lambda_{q}$ (for $q=17,21,25$ and 1) as a function of the drive laser chirp in Fig.5.6, now with $\delta \lambda_{q}$ given as the shift relative to the harmonic center wavelength at zero chirp for a gas pressure of 6 mbar.

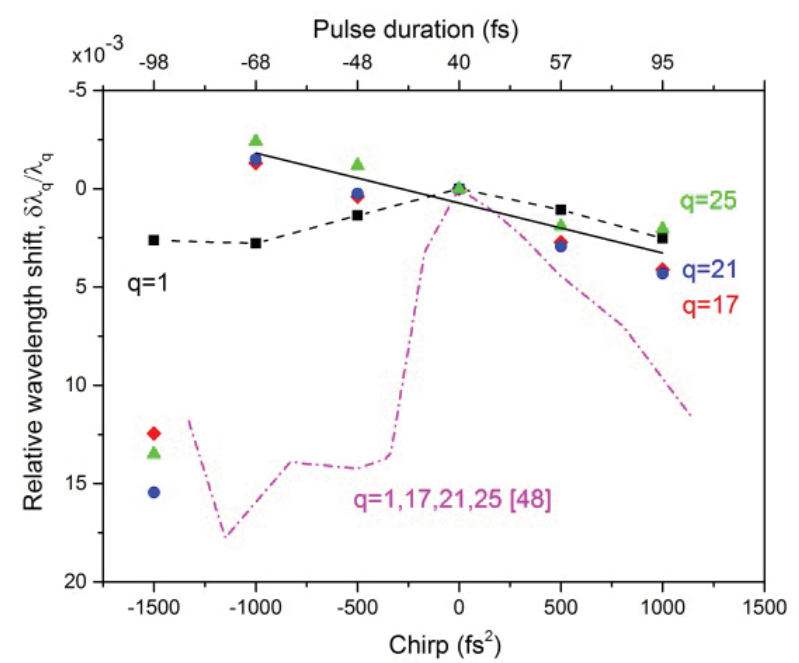

Figure 5.6: Relative wavelength shift of the $17^{\text {th }}, 21^{\text {st }}$ and $25^{\text {th }}$ harmonic orders as a function of pulse duration (chirp) with respect to harmonic peak wavelength at zero chirp (Ar gas pressure of 6 mbar). The dashed line indicates the relative wavelength shift of the drive laser, the dashed dotted line is reproduced from [48]. The sign of the pulse duration corresponds to the sign of the applied drive laser chirp.

Compared to our results indicated by the straight-lines, it can be seen that the degree of shift obtained in [48] is higher which we address to their using of much higher Ar gas pressure. However, with 80 mbar of Ar, which is a factor of 13 more than in our case, they obtained only 2.4 -times $(0.012 / 0.005)$ more wavelength shift at +95 fs. This comparison shows that $\mathrm{HH}$ spectral control in a thin capillary is yielding higher absolute numbers but on the other hand, wavelength-shifting is about a factor of 5.5 more efficient in a wide capillary when normalizing it to the pressure. This observation indicates that in our experiment the second and the third mechanisms, which are based on the single-atom response, are larger than the contribution of the first mechanism. This is also supported by experiments in the thin capillary, such as apparent in the data of Ref. [48], where the relative wavelength shift, $\delta \lambda_{q} / \lambda_{q}$, for all harmonic orders is the same as for the drive laser, which implies that there the main mechanism is ionization induced blue shift. This is confirmed qualitatively because, regardless of the sign of the chirp, the harmonics in [48] (the dashed-dotted curve in Fig5.6) show only a reduced blue shift (maximum 
value of +0.018 ) relative to their maximum shift at zero chirp setting. In our case, with respect to the blue shift at zero chirp the harmonics are found shifted in both directions, red and blue shifted (from -0.0025 to +0.015 ) following the sign of the chirp.

\subsection{Conclusions}

We have shown that waveguided high-harmonic generation (HHG) at elevated drive energy via using a wide capillary can be wavelength tuned, and we compare the tuning with that in a thin, standard capillary. Applying elevated drive energy, we have increased the harmonic output approximately three-fold using a three-times wider capillary. With experimental parameters much different from a standard capillary, specifically, a much lower gas pressure at which phase matching occurs, we have investigated the spectral control of HHG as a function of gas pressure, and as a function of the drive laser pulse duration that was adjusted via a linear chirp. The relative shift of the high-harmonics, $\delta \lambda_{q} / \lambda_{q}$, are found to range from 0 to 0.0075 via increasing the gas pressure, and from -0.0025 to +0.015 following the sign of the chirp. We found the wavelength shift is based not only on ionization induced blue-shift as reported for a standard capillary, but that other non-adiabatic mechanisms provide significant contributions as well. The results shows that in spite of rather different operational parameters, the wavelength shifting of high-harmonics in such capillaries can be as effective as with a standard capillary. The increased output pulse energy and the significantly lower electron densities (due to much lower gas pressure) avoiding complicated nonlinear propagation effects for the drive laser, appears attractive for applications that require spatially and spectrally controlled XUV radiation. 


\section{6 \\ Fabrication and characterization of XUV transmission grating}

\subsection{Introduction}

The wavelength band from the vacuum ultra-violet (VUV, 200 to $10 \mathrm{~nm}$ ) to the soft$\mathrm{x}$-ray range (SXR, 20 to $2 \mathrm{~nm}$ ), also referred to the XUV band, is highly relevant for a number of important applications and research fields. These include fluorescence analysis of materials in the SXR band [125], high resolution photolithography in the XUV [126, 127], research at free electron lasers in the SXR band [128], and XUV space research [129]. The spectral analysis of radiation in these wavelength ranges requires dispersive elements with adequate spectral resolution.

XUV spectrometers based on transmission gratings [130] are of significant relevance because they offer a number of important advantages, such as compactness and ease in alignment at normal incidence, compared to reflective gratings which use grazing incidence. Due to the free-standing geometry, light is transmitted directly through the open space between the lines of the grating. Therefore, the transmitted power spectral density is approximately independent of the wavelength, which eases calibration. Combining transmission gratings with, e.g., back illuminated CCD cameras, compact and easy-to-operate XUV spectrometers can be built.

To accommodate for a specific application, the line density of a transmission grating needs to be tailored to provide sufficient spectral resolution in the wavelength range of interest. For instance, to characterize an XUV light source emitting at around $13.5 \mathrm{~nm}$ or $6.8 \mathrm{~nm}$, it turns out that extremely dense gratings near current nanofabrication limits are required due to the short wavelength. Specifically, grating periods in the order of $100 \mathrm{~nm}$ (i.e., 10,000 lines per $\mathrm{mm}$ ) are required to achieve a resolution of at least a few tenths of $\mathrm{nm}$. To maximize the free spectral range, undesired diffraction orders need to be suppressed by choosing an appropriate space-to-period ratio. This imposes strict demands regarding the fabrication accuracy of the space-to-period ratio. Furthermore, it needs to be considered that fabrication of narrow lines implies that the thickness of the lines is small as well. This can lead to an undesired residual transmission of light through the lines, which reduces the contrast of the grating transmission function. As a result, the fabrication of free-standing transmission gratings with high line density, accurate space-to- 
period ratio and appropriate line thickness is extremely challenging even with stateof-the-art nanolithography. The current fabrication methods for high-density transmission gratings include electron beam lithography (EBL) [131] and interferometric lithography (IL) $[132,133]$. However, EBL provides a relatively low throughput, while IL has a limited resolution.

This chapter describes the first fabrication and optical characterization of freestanding, high-line-density gratings based on UV-NIL. VUV based nano imprint lithography (UV-NIL) is applied using a lift-off and back etch process to fabricate the gratings. This fabrication method has been shown to obtain high resolution and highly reproducible nanometer-scale structures [134]. Furthermore, the resolution is comparable to the high resolution of electron beam lithography, however, with much higher throughput in fabrication. Essential for the development of the overall grating fabrication process and the associated costs is the relatively fast and straightforward parallel printing nature of NIL. The latter allows fabrication of many slightly different gratings in a single step, which facilitates the optimization for the various lift-off and back etch processes with the goal to improve the quality of the fabrication process. Once optimized, this feature also allows the fabrication of a set of gratings with different line densities in a single batch. In conjunction with Industrial Focus Group XUV Optics group, the fabrication of these gratings was carried out by Dr.-Ing. Boris Vratzov.

The optical characterization is important for quantifying the relative strength of higher diffraction orders, particularly the second order, which depends on imperfections in fabrication that might lead to, e.g., an asymmetric space-to-period ratio. Such imperfections in grating features cannot be observed via a morphological analysis alone, such as based son Scanning Electron Microscopy (SEM). An optical characterization is also important for evaluating the spectral resolution that can be achieved. The characterization requires XUV sources with appropriate spatial and spectral coherence. Here we use the XUV sources. The first is based on highharmonic generation (HHG) describe in this thesis, which offers a high spatial coherence and a well-structured spectrum comprising a distinct and known set of wavelengths across the entire range from $17.5 \mathrm{~nm}$ to $40.5 \mathrm{~nm}$. The second source is based on a Xenon plasma emitting in the range around $13.5 \mathrm{~nm}$ which was carried out in ASML. The experimental measurement and analysis of all spectra, as well as comparison to theory were conducted by myself.

In the following Sect. 6.2, we describe the experimental setup in which the gratings were characterized, and we present the design criteria and the fabrication method for the transmission gratings. In Sect. 6.3, we discuss the results obtained with the optical characterization. Special emphasis is put on the comparison of gratings that differ in their space-to-period ratio but possess the same grating period, and on measuring the experimental resolution for a comparison with the theoretically expected values. Finally, we conclude in Section 3.7 the major experimental results and findings. 


\subsection{Experimental details}

\subsubsection{Transmission grating spectrometer}

Figure 6.1(a) gives an overview of the transmission grating spectrometer setup, which comprises an incident beam (here from a high-harmonic source or a plasma source), an entrance slit and a transmission grating. The diffracted light falls on a detector, which is the chip of a back-illuminated CCD camera (Andor, DO420-BN). The advantages of such setup are that the spectral range and spectral resolution can be adjusted easily via the distance from the grating to the detector, that a relatively high first-order diffraction efficiency is achieved, and that the diffraction efficiency is nearly independent of the wavelength. Figure 6.1(b) illustrates the front view and cross section of one of the transmission gratings. It can be seen that the overall grating dimension is $4 \mathrm{~mm} \times 1 \mathrm{~mm}$, divided up into 2666 separate areas (five areas are shown schematically), each being $1200 \mathrm{~nm}$ high, separated by $300 \mathrm{~nm}$ wide support bars. The support bars are required for the mechanical stability of the grating in view of its large area. The actual line structure of the grating shown in Fig.1(c) consists of equidistant parallel grating lines of $b=50 \mathrm{~nm}$ width, spaced by $a=50$ $\mathrm{nm}$, to yield a grating period of $d=100 \mathrm{~nm}$. The cross section of the grating lines consists of a $200 \mathrm{~nm}$ thick $\mathrm{Si}_{3} \mathrm{~N}_{4}$ layer, a $70 \mathrm{~nm}$ thick Au layer and a $12 \mathrm{~nm}$ thick $\mathrm{Cr}$ layer, carried on a $380 \mu \mathrm{m}$ thick Si wafer.

In the following, to prepare for comparison with experimental data, we briefly recall some basic relations describing diffraction at a grating. We assume that the grating-detector distance, $R$, is much larger than the space between two grating lines, $a$, which is fulfilled here. For a plane wave that is normally incident on the grating, the diffraction angle for a wavelength $\lambda$ is given by the well-known grating equation

$$
d \sin \alpha=m \lambda,
$$

where $d$ is the grating period, $\alpha$ is the diffraction angle and $m$ is the diffraction order. As can be seen from Eq. 6.1, when an incident beam with a wide range of wavelengths is incident on the grating, the spectra belonging to neighboring orders may partially overlap. The spectral width across which overlapping does not occur is known as the free spectral range. To maximize the free spectral range, the power diffracted into orders bigger than one is desired to be minimal. A limitation of the spectral range that can be observed towards long wavelengths, thereby limiting the free spectral range as well, is given by the detector size and geometry as

$$
\lambda_{\max }=\frac{d}{m} \sin \left[\tan ^{-1}\left(\frac{D}{R}\right)\right],
$$


where $D$ is the transverse dimension of the CCD chip and where $R$ is the gratingdetector distance.

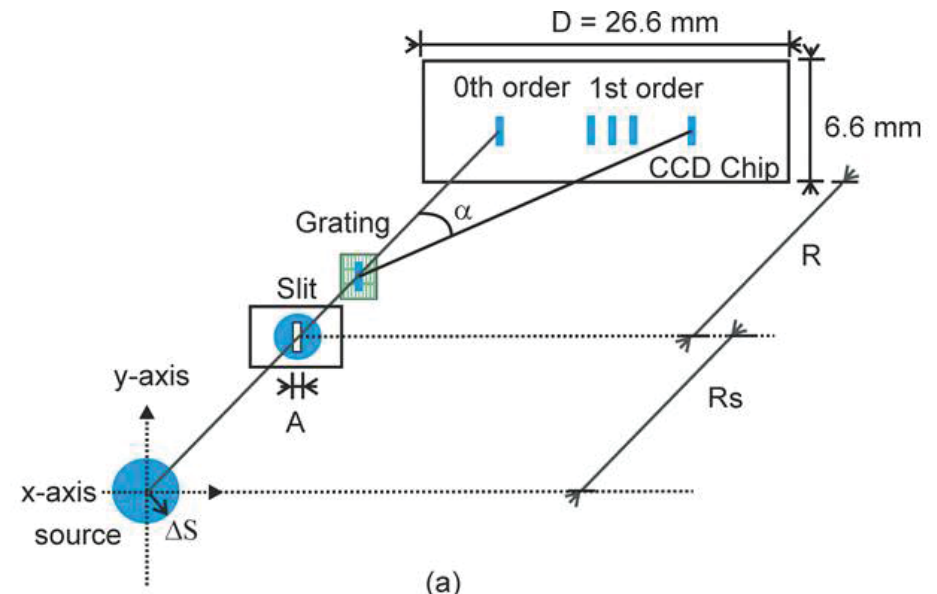

Front view grating

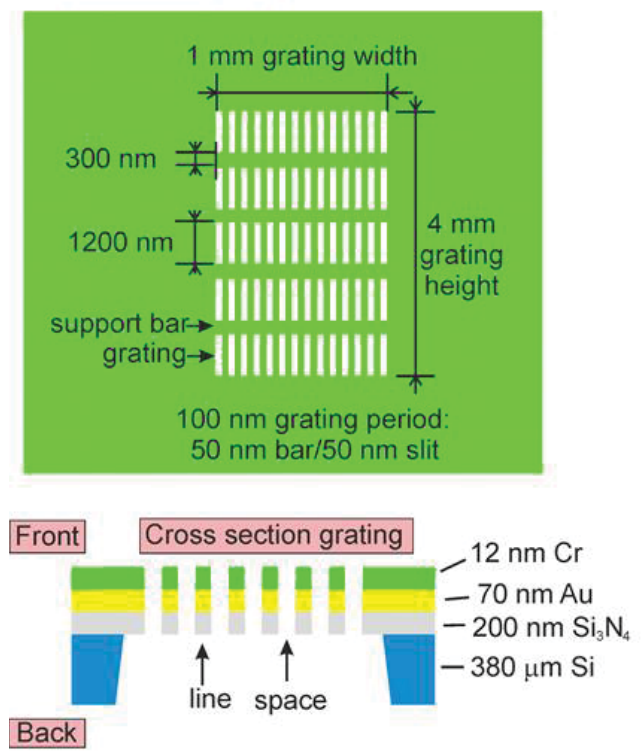

(b)

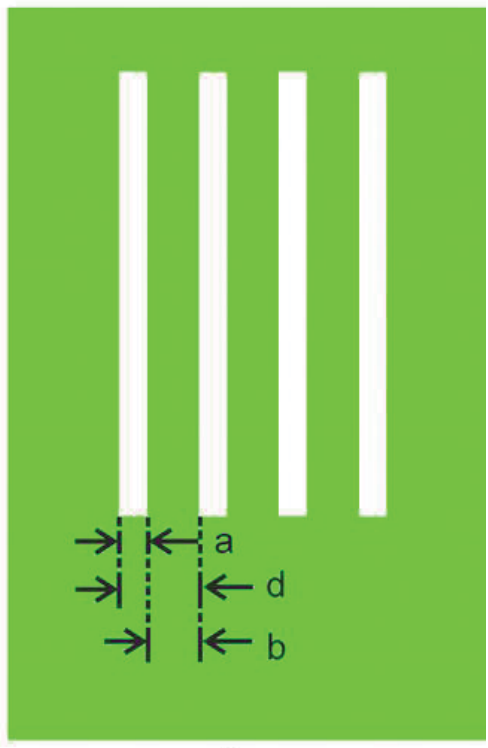

(c)

Figure 6.1: (a) Transmission grating spectrometer setup, (b) enlarged view of a transmission grating with a density of 10,000 lines $\mathrm{mm}^{-1}$, (c) definition of the grating parameters $d$ (period), $a$ (space) and $b$ (line width).

To cover the part of the XUV band that is of interest here, i.e., the wavelength range to be covered by our HHG source (from $17.5 \mathrm{~nm}$ to $40.5 \mathrm{~nm}$ ) and the range to be covered by the Xe plasma source $(10 \mathrm{~nm}$ to $17 \mathrm{~nm})$, a free spectral range from 10 $\mathrm{nm}$ to $40.5 \mathrm{~nm}$ is required. For our setup, where $D=26.6 \mathrm{~mm}$ and $d=100 \mathrm{~nm}$ (with 
$m=1$ ) we have chosen $R=60 \mathrm{~mm}$, which yields $\lambda_{\max }=40.5 \mathrm{~nm}$. Importantly, with this combination of incident wavelengths, grating period and geometry $(D / R)$, it should become possible to observe the first-order and second-order diffraction simultaneously for wavelengths between $17.5 \mathrm{~nm}$ and $20.25 \mathrm{~nm}$. Thereby the second-order diffraction efficiency and the degree of suppression of second-order diffraction $v s$. grating geometry (i.e. space-to-period ratio) can be easily quantified, as will be described in detail below.

To calculate the efficiency for the various diffraction orders, we begin with considering opaque grating lines and use a model [135] based on Kirchhoff's diffraction theory in the Fraunhofer approximation [136]. We note that considering the lines as fully opaque, as if made from a perfect metal, is done here only as an approximation. To account for the finite transmission of real metals in the XUV, we later replace the approximation and also model the grating with partially transparent lines (Eq.6.5). The Fraunhofer approximation can be expressed as the conditions $\frac{a^{2}}{R \lambda} \ll 1$ and $\frac{a^{2}}{R_{S} \lambda} \ll 1$, which are fulfilled here because the grating slit width, $a$, is larger than or comparable with the wavelength, $\lambda$, but is very small compared to the distances $R_{s}$ and $R$ [see Fig. 6.1]. The model is further simplified by using a onedimensional analysis with the assumption that the grating consists of very long equidistant parallel slits. We hereby neglect the secondary interference pattern introduced by the grating support bars that extends vertically in Fig. 6.1(a). With these approximations, the diffraction efficiency, $\eta_{m}$, of the $m^{\text {th }}$ order is given by [135]

$$
\eta_{m}=\left[\frac{\sin \left(m \pi \frac{a}{d}\right)}{m \pi}\right]^{2} .
$$

The ratio of power in the $m^{\text {th }}$ order with regard to the first order is then obtained as

$$
T_{m / 1}=\frac{\eta_{m}}{\eta_{1}}=\left(\frac{\sin \left(m \pi \frac{a}{d}\right)}{m \sin \left(\pi \frac{a}{d}\right)}\right)^{2} .
$$

From Eq. 6.4 it can be seen that choosing a symmetric grating characterized by a space-to-period ratio, $\mu=a / d=0.5$, we obtain $T_{2 / 1}=0$. This corresponds to the ideal case that the $2^{\text {nd }}$ order is fully suppressed, thereby effectively doubling the free spectral range. What can be seen from Eq. 6.4 as well, valid when the grating lines are completely opaque, is that the calibration of the optical power spectral density of the grating is straightforward because the diffraction efficiency of the first and all higher orders would be independent of the wavelength. However, the lines of the 
grating investigated here might not be completely opaque due to their small thickness which is the result of a limited aspect ratio in etching narrow lines. In the wavelength range considered here the absorption lengths for the materials used $\left(\mathrm{Si}_{3} \mathrm{~N}_{4}, \mathrm{Au}\right.$, and $\left.\mathrm{Cr}\right)$ lie in the range between 1 and $10 \mathrm{~nm}$. The residual transmission of light which propagates through the grating lines, and the wavelength dependent phase shift of that light, will lead to a diffraction efficiency that is not spectrally flat, which would have to be taken into account in an accurate spectral calibration of the grating. In order to predict in how far such effects would play a role for the gratings with our dimensions and to justify additional fabrication steps that could avoid these effects, we look at the first-order diffraction efficiency versus wavelength for a grating with partially transmitting lines [135]

$$
\eta_{m}(\lambda)=\left(\frac{\sin (m \pi \mu)}{m \pi}\right)^{2}\left[1+\exp \left(-\frac{4 \pi n_{2} z_{0}}{\lambda}\right)-2 \exp \left(-\frac{2 \pi n_{2} z_{0}}{\lambda}\right) \cos \left(\frac{2 \pi\left(n_{1}-1\right) z_{0}}{\lambda}\right)\right] .
$$

Here $n=n_{1}+i n_{2}$ is the complex, wavelength-dependent refractive index of the grating line material, $z_{0}$ is the thickness of the grating lines and the $\mu$ is the space-toperiod ratio defined above. The red curve in Fig.6.2 shows the first-order diffraction efficiency, calculated with Eq. 6.5 for a symmetric $(\mu=0.5) \mathrm{Si}_{3} \mathrm{~N}_{4}$ grating with $z_{0}=$ $200 \mathrm{~nm}$ thick lines, which we consider a safe but lower limit of thickness in terms of fabrication. The complex index of $\mathrm{Si}_{3} \mathrm{~N}_{4}$ is taken from [100]. It can be seen that the diffraction efficiency lies around an average value of $10 \%$ and that there is indeed quite some spectral variation, particularly in the wavelength range between 13 and $30 \mathrm{~nm}$. To predict whether this undesired effect can be minimized with an additional absorbing layer, we also calculated the spectral response of the $\mathrm{Si}_{3} \mathrm{~N}_{4}$ grating for the case that a more strongly XUV absorbing $\mathrm{Au} / \mathrm{Cr}$ bilayer of $70 / 12 \mathrm{~nm}$ is deposited on top. The calculation is based on Eq. 6.5 again, where the sequential transmission through the materials $\left(\mathrm{Si}_{3} \mathrm{~N}_{4}, \mathrm{Au}, \mathrm{Cr}\right)$ was modeled via adding their respective absorbance in the exponentials and via adding their respective phase shifts in the cosine argument. The complex index data were taken from [100]. The first-order efficiency of this grating with increased line opacity is shown in Fig. 6.2 as the black curve. It can be seen that a much more uniform spectral response is obtained with only a $2 \%$ variation around the $10 \%$ average. 


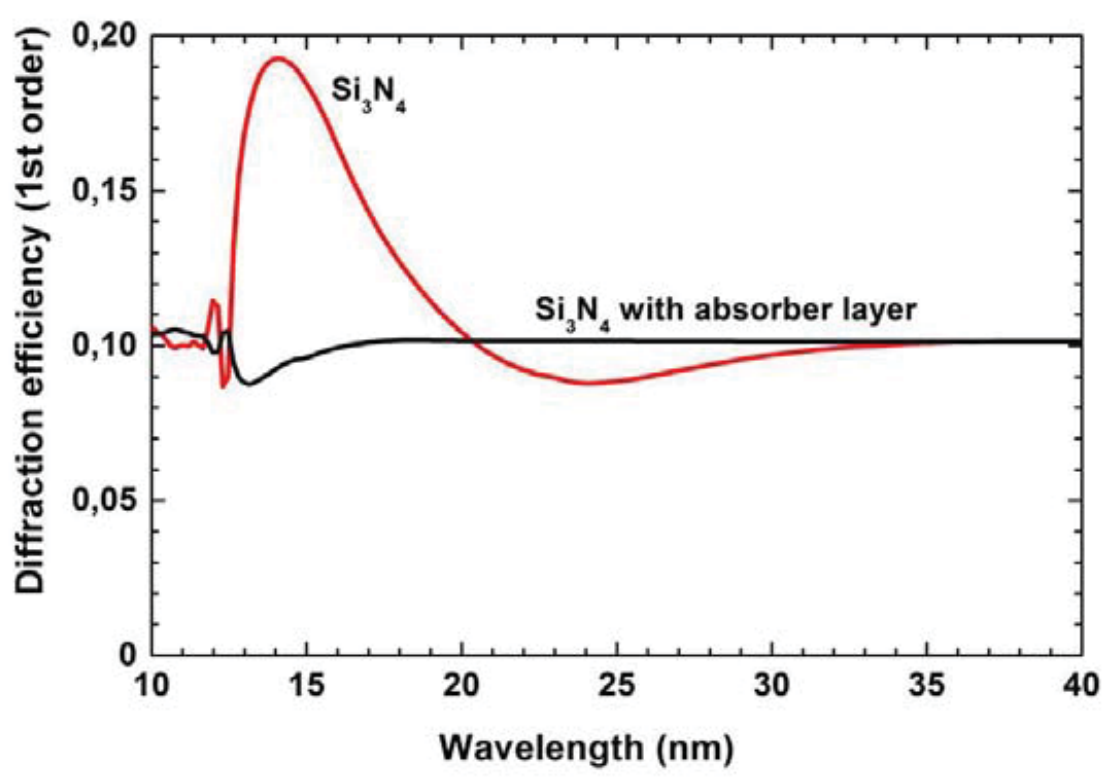

Figure 6.2: Calculated spectral variation of the first order diffraction efficiency for a symmetric $(\mu=$ 0.5 ) grating with 200 -nm thick $\mathrm{Si}_{3} \mathrm{~N}_{4}$ lines (red curve) and the same grating with an extra $70 \mathrm{~nm} \mathrm{Au}$ layer and an extra $12 \mathrm{~nm}$ Cr layer deposited on the lines (black curve).

As another important characteristic we consider the spectral resolution [137],

$$
\Delta \lambda_{t h}=\left\{\left[\frac{d}{m}\left(\frac{\Delta S+A}{R_{s}}\right)+\frac{d}{m}\left(\frac{K}{R}\right)\right]^{2}+\left(\frac{\lambda d}{m A}\right)^{2}\right\}^{1 / 2}
$$

which is determined to a first part by the geometrical resolution (first square term) and to a second part by the dispersive resolution of the grating (second square term). The geometrical resolution takes into account the finite diameter of the source, $\Delta S$, the width of the aperture, $A$, the width of a CCD-pixel, $p$, the distance between the source and the grating, $R_{s}$, and the distance between the grating and the detector, $R$. We note that $K$ corresponds to the full width at half-maximum for the convolution of $A$ across $p$ [138]. Equation 6.6 shows that the achievable spectral resolution depends not only on the parameters of the spectrometer setup, but also on the source, via $\Delta S$ and $R_{s}$, which will be discussed later in Sect. 6.3.

\subsubsection{Fabrication of gratings}

In the previous section, we have given an example of the desired parameters for the 10,000 lines $\mathrm{mm}^{-1}$ gratings which are based on fabrication with a $200 \mathrm{~nm}$ thick grating lines made from $\mathrm{Si}_{3} \mathrm{~N}_{4}$ with an $\mathrm{Au} / \mathrm{Cr}$ bilayer of $70 / 12 \mathrm{~nm}$ on top and a space-to-period ratio of $\mu=0.5$. Here we describe the fabrication of such gratings using the following lithographic technique. Firstly, a layer of $200 \mathrm{~nm} \mathrm{Si}_{3} \mathrm{~N}_{4}$ is 
deposited on a $400 \mu \mathrm{m}$ thick Si wafer. The wafer material is to be removed later except for a frame and the $300 \mathrm{~nm}$ wide bars to support a free-standing membrane. $\mathrm{Si}_{3} \mathrm{~N}_{4}$ is chosen because this material allows the fabrication of high quality membranes with low and well-controlled internal stress. For defining the grating patterns, we apply UV based nano imprint lithography (UV-NIL) using a single imprint template comprising 21 individual gratings placed over a total area of $16 \mathrm{x}$ $16 \mathrm{~mm}^{2}$. The different gratings possess line densities of 500, 780, 1000, 1500 and $1850 \mathrm{~mm}^{-1}$, and from 2000 to $10,000 \mathrm{~mm}^{-1}$ in steps of $1000 \mathrm{~mm}^{-1}$. Each grating has a free-standing area of $4 \mathrm{~mm}$ height and $1 \mathrm{~mm}$ width. The imprints were carried out on an Imprio 55 device (Molecular Imprints, Inc.).

The fabrication process is shown in Fig. 6.3. First, the substrate is spin-coated at $3000 \mathrm{rpm}$ with a $100 \mathrm{~nm}$ thick water solvable transfer layer (UL-300 from Micro Resist Technology $\mathrm{GmbH}$ ) and then baked at $150 \mathrm{C}$ for $120 \mathrm{~s}$ on a proximity hot plate. In the imprint machine, the imprint resist is dispensed on top of the transfer layer [Fig. 6.3(a)]. The template is then pressed into the imprint resist with a force of $3 \mathrm{~N}$ at room temperature. After spreading, the imprint resist is cured with a $3 \mathrm{~s} \mathrm{UV}$ exposure through the transparent template while the template is still in contact. After that, the template is detached leaving its negative shape in the hardened imprint resist. The patterned layer shows a feature height of $100 \mathrm{~nm}$ and a residual layer of $35 \mathrm{~nm}$ [Fig. 6.3(b)]. In the next step, a planarization layer of $160 \mathrm{~nm}$ hydrogen silsesquioxane (HSQ), XR-1541 6 (from Dow Corning), is deposited by spin coating on top of the imprinted feature [Fig. 6.3(c)]. After getting a planarized surface the etch-back of the top layer of HSQ, followed by a pattern transfer through the residual imprint resist and transfer layer [Fig. 6.3(d)], is carried out using a two-step Reactive Ion Etching (RIE) process in fluorine and oxygen-based plasma. The planarization and etch back RIE process offers very high critical dimension (CD) control as well as a small sidewall undercut as needed for a lift-off process. An XUV absorber layer of $\mathrm{Au}(70 \mathrm{~nm})$ followed by an etch mask layer of $\mathrm{Cr}(12 \mathrm{~nm})$ is deposited by metal evaporation using an electron beam deposition tool [Fig. 6.3(e)]. Then, lift-off to form the top absorber layer and the etch mask for patterning the $\mathrm{Si}_{3} \mathrm{~N}_{4}$ layer is carried out by dipping the sample into deionized water in an ultrasonic bath [Fig. 6.3(f)]. The openings for the single gratings of $1 \mathrm{~mm} \times 4 \mathrm{~mm}$ on the back side are defined by optical lithography followed by a fluorine-based RIE of the $\mathrm{Si}_{3} \mathrm{~N}_{4}$ layer on the backside and a 4 hours wet etch process in potassium hydroxide $(\mathrm{KOH})$ removing the bulk silicon and stopping on the top $\mathrm{Si}_{3} \mathrm{~N}_{4}$ layer [Fig. 6.3(g)]. The final step in the fabrication of the grating is to etch through the $\mathrm{Si}_{3} \mathrm{~N}_{4}$ membrane using the Cr layer as a etch mask in the same fluorine-based RIE [Fig. 6.3(h)]. 
(a)

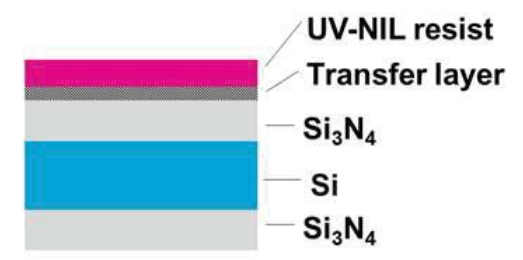

(c)

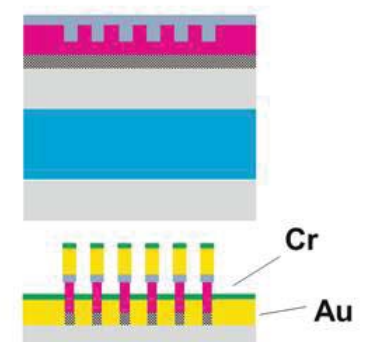

(e)

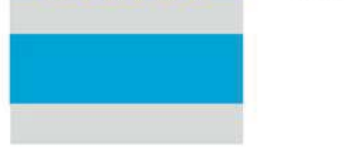

(g)

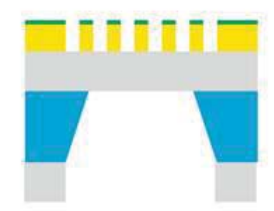

(b)

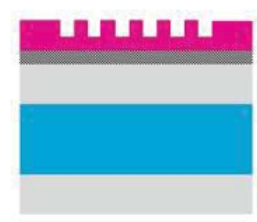

(d)

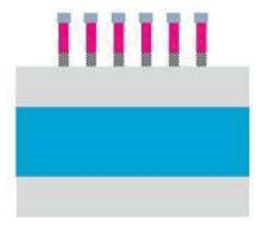

(f)

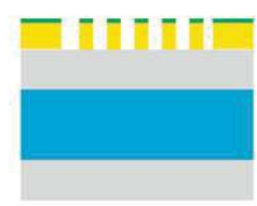

(h)

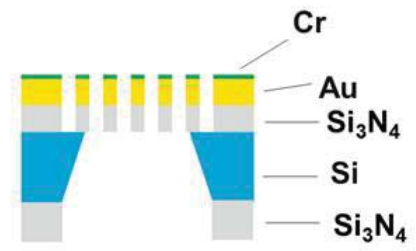

Figure 6.3: Process flow for fabrication of the free-standing gratings: (a) deposition of $\mathrm{Si}_{3} \mathrm{~N}_{4}$ and imprint resists on a Si wafer, (b) UV-Nano Imprint Lithography (UV-NIL) for high resolution pattern definition, (c) planarization and etch back of the top layer by Reactive Ion Etching (RIE), (d) pattern transfer down to the $\mathrm{Si}_{3} \mathrm{~N}_{4}$ by RIE with excellent $\mathrm{CD}$ control and a small undercut for a lift-off process, (e) deposition of an $\mathrm{Au}$ absorber layer and $\mathrm{Cr}$ etch mask by evaporation, (f) lift-off to form the top absorber layer and etch mask, (g) back side optical lithography and subsequent patterning of $\mathrm{Si}_{3} \mathrm{~N}_{4}$ by RIE and Si by wet etching, (h) grating definition by RIE etch into $\mathrm{Si}_{3} \mathrm{~N}_{4}$.

For the highest line density of 10,000 lines $\mathrm{mm}^{-1}$, we have fabricated five different gratings to which we refer as G1, G2, G3, G4 and G5 with a grating spaceto-period ratio of $\mu=0.45,0.50,0.50,0.50$ and 0.55 , respectively. The motivation for fabrication of multiple gratings with the same $\mu$-values (G2, G3, G4) is to obtain a measure for fabrication reproducibility. Different $\mu$-values (G1, G2/G3/G4, G5) have been fabricated as well, in order to increase the chances for realizing an optimized grating with a maximally suppressed second diffraction order. In spite of intrinsic fabrication errors, this approach is relatively cost effective with our NIL fabrication method because all of the five gratings are available on a single imprint template and can be fabricated all at once in a single process.

In order to find out how close to specifications our gratings are, we started characterizing the gratings with recording SEM images. Fig. 6.4 shows, as an example, a SEM image of grating G3 (design goal $\mu=0.5$ ). It can be seen that the grating is highly periodic as desired, and that the width of the grating lines is rather 
uniform. The SEM images of the other gratings (G1, G2, G4, G5) exhibit similarly high periodicity and uniformity.

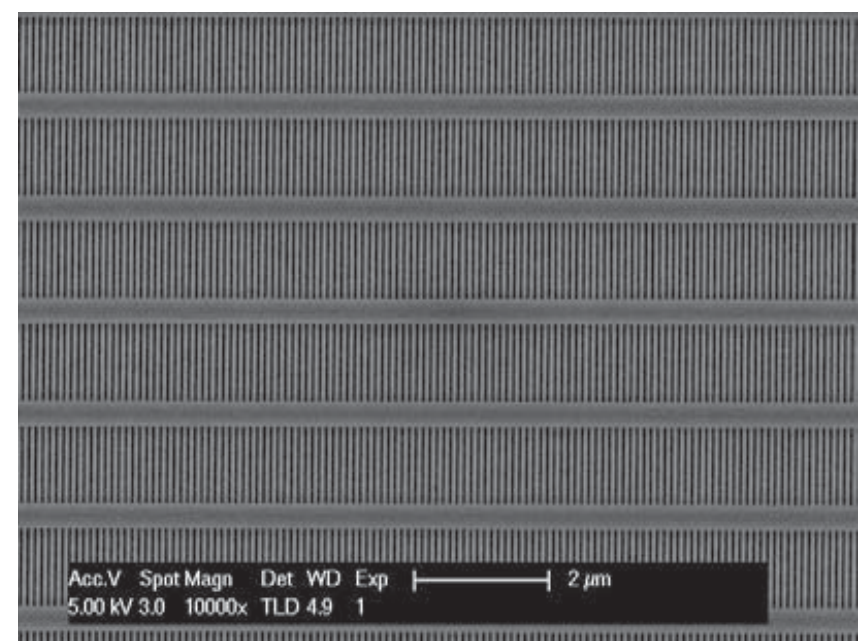

Figure 6.4: Scanning electron microscopy image for the 10,000 lines $\mathrm{mm}^{-1}$ grating G3 (design for $\mu=$ $50 / 50)$.

A quantitative evaluation of the fabricated average space-to-period ratio, $\bar{\mu}$, of the gratings is carried out by measuring 30 different sampling points (space-to-period ratios) from each of their corresponding SEM image. The evaluation reveals that, for all the gratings, $\bar{\mu}$ is close to the intended design ratio, $\mu$, with a standard deviation around $\sigma=0.04$, as shown in Fig. 6.5. To obtain a measure for fabrication reproducibility of different gratings with the same design goal, we calculated the standard deviation from the measured $\bar{\mu}$-values of the gratings G3, G4 and G5 ( $\mu=$ 0.5 ). This yields a value of 0.02 , corresponding to a reproducibility of $4 \mathrm{~nm}$ for the width of the grating lines.

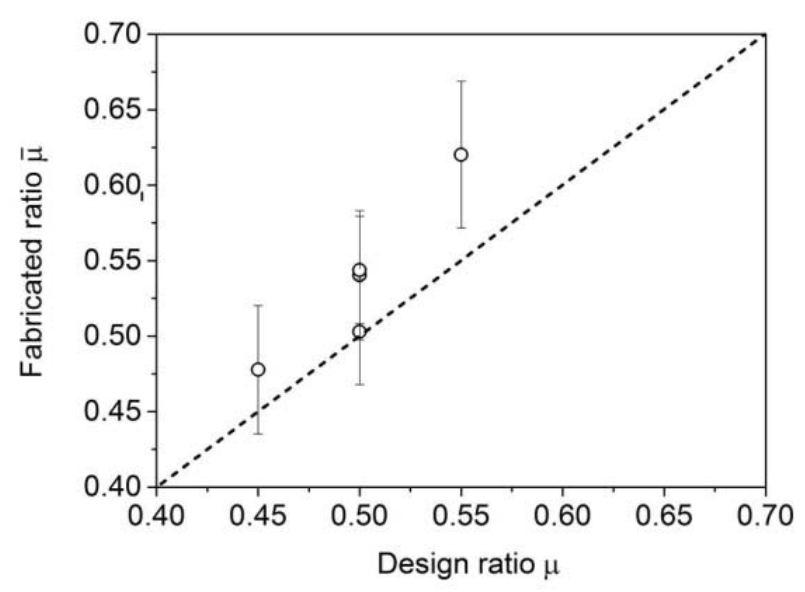

Figure 6.5: Fabricated average space-to-period ratios, $\bar{\mu}$, determined from the SEM images for the gratings $\mathrm{G} 1, \mathrm{G} 2 / \mathrm{G} 3 / \mathrm{G} 4$ and $\mathrm{G} 5$ versus the intended design ratios, $\mu$, of $0.45,0.5$ and 0.55 . The full length of the error bars is taken as $2 \sigma$, where $\sigma$ is the standard deviation measured in the SEM images for each grating. The dashed line indicates the ideal case of zero fabrication error. 


\subsection{XUV optical characterization and discussion}

For an optical characterization we used two different XUV sources. The first is based on high-harmonic generation (HHG) which is available in table-top scale and easily accessible as compared to, e.g., a synchrotron source. The HHG output possesses an excellent spatial coherence resulting in a low divergence beam $(\sim 0.1$ $\mathrm{mrad}$ ), and it offers a well-structured spectrum comprising a distinct set of known wavelengths. The radiation is generated via a highly nonlinear optical process that produces ultrashort pulses with a broad spectrum including XUV radiation, obtained by focusing ultrahigh intensity infrared laser pulses into a gaseous medium, typically a noble gas $[45,78]$. The experimental setup for the XUV source has been described in Chap.4, but here we recall the setup for convenience which is schematically shown in Fig. 6.6. To drive the HHG, we employ a pulsed Ti:Sapphire infrared (IR) laser with $780 \mathrm{~nm}$ center wavelength at $1 \mathrm{kHz}$ repetition rate (Legend Elite Duo HP USP, Coherent Inc.). The duration of the IR laser pulses is 39 fs with a pulse energy of $6.5 \mathrm{~mJ}$. A $67 \mathrm{~mm}$ long capillary-waveguide with a $508 \mu \mathrm{m}$ wide inner diameter is mounted in a vacuum chamber and is filled with Ne gas at 40 mbar. For mode matching the drive laser beam to the lowest order waveguiding capillary mode, we use a focusing mirror with a focal length of $2.5 \mathrm{~m}$. Taking into account the coupling efficiency of the IR laser radiation into the capillary, the peak intensity launched into the waveguide is $2.6 \times 10^{14} \mathrm{~W} / \mathrm{cm}^{2}$. The cut-off wavelength of HHG, which is the shortest wavelength that can be generated [34], decreases with increasing drive laser intensity and ionization potential of the used type of noble gas. In order to observe the first-order and second-order diffraction simultaneously for wavelengths between $10 \mathrm{~nm}$ and $20.25 \mathrm{~nm}$, we chose to use the noble gas Ne due to its relatively high ionization potential, $21.6 \mathrm{eV}$. With the cut-off law [34], we determine theoretically that the obtainable cut-off wavelength from the high-harmonic source is $17.5 \mathrm{~nm}$ $\left(45^{\text {th }}\right.$ harmonic order), which is suitable for the experiments. As was describe in Section 4.4, behind the capillary, we let the high-harmonic and drive laser beams copropagate over a distance of $9 \mathrm{~m}$, for reducing the drive laser intensity by diffraction. The drive laser beam is then blocked by a set of two Aluminum (Al) filters placed in series, each of them $200 \mathrm{~nm}$ thick. The XUV radiation is sent to the transmission grating spectrometer [see Fig. 6.1(a)] for spectral measurements. The Al filters also act as a bandpass for XUV radiation, limiting the spectral transmission of the path to the CCD detector to a range from $16 \mathrm{~nm}$ to $40 \mathrm{~nm}$. This range is, however, sufficient to detect all the relevant output wavelengths of the harmonic source. 


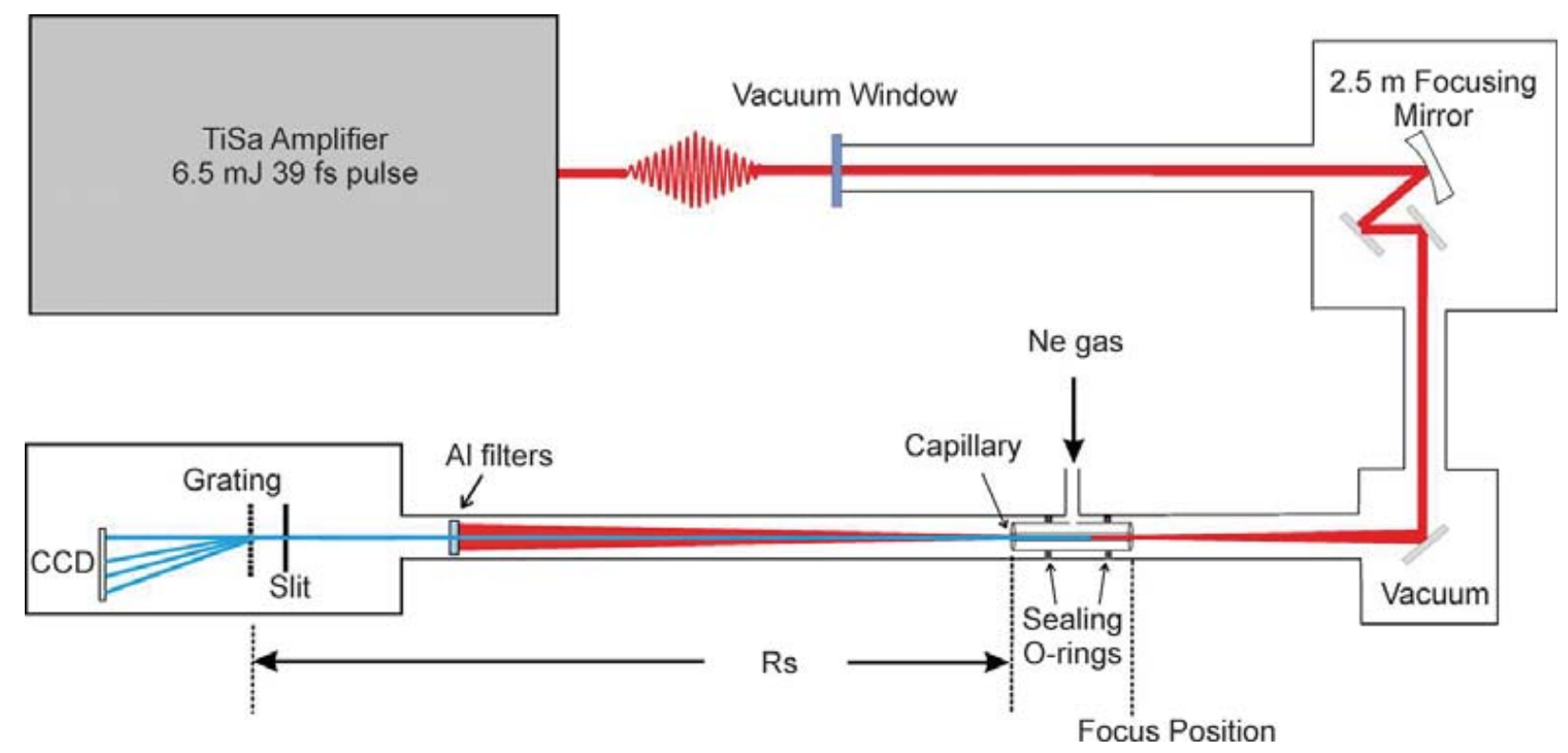

Figure 6.6: Table-top XUV source based on high-harmonic generation (HHG) with a Ne-filled capillary.

For a first step of optical characterization of the gratings, we have recorded series of spectra of the $\mathrm{HH}$ beam, using the five different gratings G1 to G5. Figure 7(a) shows an example of such spectral measurement, in this case performed with G3. The measurement shows that the grating is resolving the various high order harmonics; that only odd harmonics are generated is a typical characteristic of such a source. The highest harmonic order that we observe in the spectrum is the $45^{\text {th }}$ harmonic order at a wavelength $17.5 \mathrm{~nm}$, which matches very well with the theoretical value for the cutoff wavelength.

With the geometry of our setup, the second diffraction order for harmonic orders lower than the $39^{\text {th }}$ is not measurable as they fall out of the detector image range ( $\lambda_{\max }$ limited by $D$ in Eq. 6.2]. However, although the intensity of the secondorder diffraction peak is small (as was actually intended via choosing $\mu=0.5$ ), there are several harmonic orders (from $39^{\text {th }}$ to $45^{\text {th }}$ ) of which we can record the first and second diffraction order simultaneously. This simultaneous appearance, resulting from our choice of suitable parameters (Ne-gas, peak intensity of $2.6 \times 10^{14} \mathrm{~W} / \mathrm{cm}^{2}$, grating period, $d=100 \mathrm{~nm}, D=26.6 \mathrm{~mm}$ and $R=600 \mathrm{~mm}$ ), is what enables to extract the ratio of $2^{\text {nd }}$ to $1^{\text {st }}$ order ratio of diffraction efficiency, $\eta_{2} / \eta_{1}=T_{2 / 1}$, and compare it with the theoretically expected value that should depend on $\bar{\mu}$. 

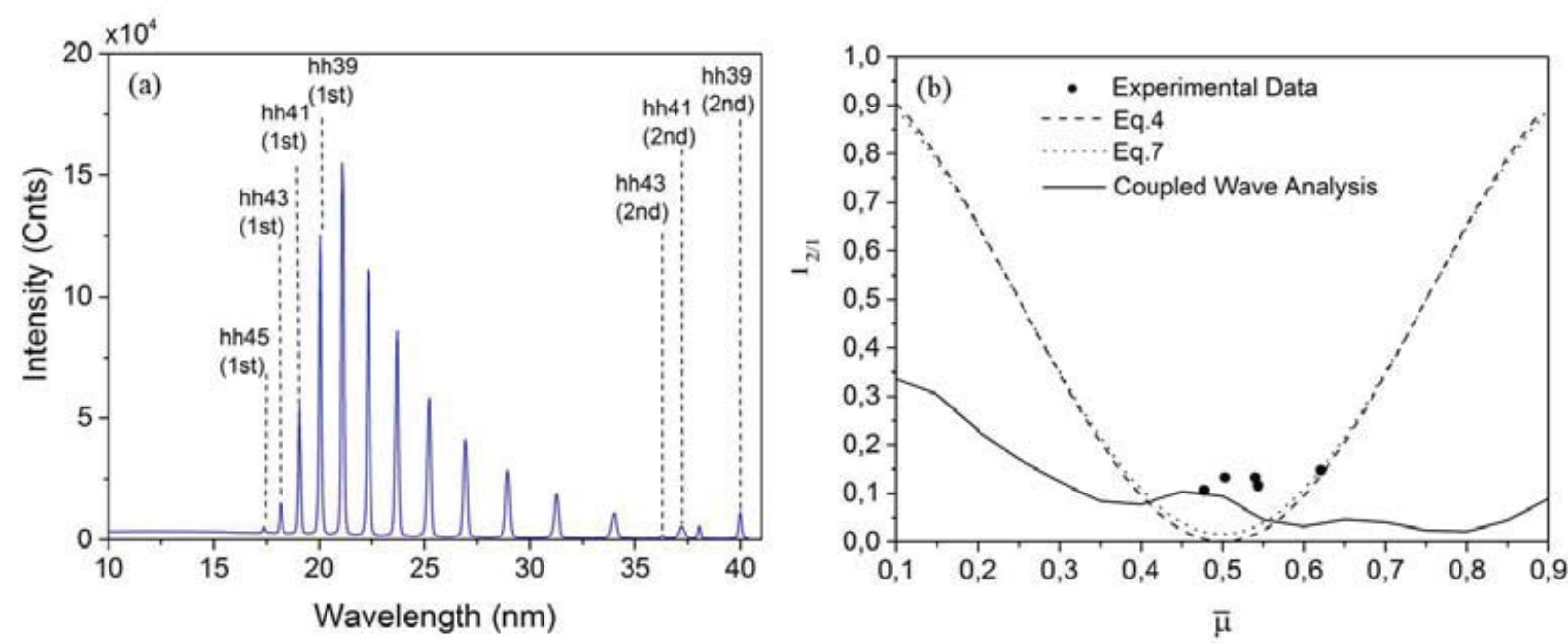

Figure 6.7: (a) The high-harmonic spectrum measured with a 10,000 lines $\mathrm{mm}^{-1}$ grating (G3). (b) Ratio of the second to first order diffraction efficiency measured at the $39^{\text {th }}$ harmonic order $(\lambda=20 \mathrm{~nm})$ for five different gratings (black circles), second to first order diffraction efficiency calculated for a onedimensional grating structure using Eq. 6.4 (dashed line) and Eq. 6.7 (dotted line), and for a twodimensional grating, i.e., taking into account the finite thickness and also layered structure of the grating lines, based on a rigorous coupled wave analysis (solid line).

The spectra recorded with the five gratings show rather similar characteristics except for the relative height of the second-order diffraction peaks. The latter is quantified in Fig. 6.7(b), where the experimental values of $T_{2 / 1}$ for the gratings (G1G5) with different $\bar{\mu}$ ratio are plotted as black circles. The values $T_{2 / 1}$ are obtained by taking the ratio of the spectrally integrated counts of the first and second diffraction orders for the $39^{\text {th }}$ harmonic order $(20.25 \mathrm{~nm})$. This order is selected because it provides the highest signal in the $1^{\text {st }}$ and $2^{\text {nd }}$ diffraction order compared to the other harmonic orders. We now compare, as a first step, the experimental values of $T_{2 / 1}$ with the simple Fraunhofer theory for a one-dimensional grating as given in Sect. 6.2. For this case we take Eq. 6.4 with $m=2$, which is plotted in Fig. 6.7(b) as the dashed curve. It can be seen that one of the experimental data points matches well with theory but that the other data deviate noticeably. To investigate whether fabrication imperfection is the cause for deviation, we have recalculated the ratio $T_{2 / 1}$, however, by taking into account the distribution of $\bar{\mu}$-values that are found in the fabricated gratings. For a quantification we have used a Gaussian distribution as weighting factor for Eq. 6.4. The fabrication error averaged efficiency ratio is then given by

$$
T_{2 / 1}(\bar{\mu})=\int_{0}^{1}\left(\frac{1}{\sigma \sqrt{2 \pi}} e^{-\frac{(\mu-\bar{\mu})}{2 \sigma^{2}}}\right)\left(\frac{\sin (2 \pi \mu)}{2 \sin (\pi \mu)}\right)^{2} d \mu,
$$

where the first bracket in the integral represents the Gaussian distribution function and the second bracket is taken from Eq. 6.4 for $m=2$. The dotted curve in Fig. 6.7(b) shows a calculation of $T_{2 / 1} v s . \bar{\mu}$ using Eq. 6.7 with $\sigma=0.04$ which is the 
standard deviation obtained from the SEM images averaged over the five available gratings. We observe that the dotted curve yields a slightly higher $T_{2 / 1}$ ratio as compared to the dashed curve, yet, having taken into account the experimental fabrication error via an averaging does not well explain the experimental $T_{2 / 1}$ values. We believe that the poor match of both the curves obtained with Eqs. 6.4 and 6.7 is due to the approximation of the gratings being one-dimensional, i.e., having a thickness of zero in the longitudinal direction. To take the finite thickness of the gratings into account, i.e., that the grating lines have a finite thickness and consist of three different material layers $\left(\mathrm{Si}_{3} \mathrm{~N}_{4} 200 \mathrm{~nm}, \mathrm{Au} 70 \mathrm{~nm}\right.$, and $\left.\mathrm{Cr} 12 \mathrm{~nm}\right)$, we have applied a Rigorous Coupled Wave Analysis (RCWA) method [139] to calculate the grating diffraction efficiencies. The RCWA is a semi-analytical method which determines the $1^{\text {st }}$ and $2^{\text {nd }}$ order diffraction efficiencies by solving Maxwell's equation in Fourier space for the incident beam which propagates through each grating line material. The calculated efficiency, $T_{2 / 1}$, is plotted as a function of $\bar{\mu}$ as the solid curve in Fig. 6.7(b). It can be seen that this solid curve shows a much better agreement with the measurement values than was obtained with Eqs. (6.4) and (6.7). We note, although $T_{2 / 1}$ is weakly wavelength dependent, that there is no significant change (less than $0.5 \%$ ) within the finite spectral bandwidth of the considered harmonic. We assume that the remaining discrepancies are due to an unknown tiltangle of the incident beam $[140,141]$ or due to a slightly trapezoidal profile [142] that may have been caused in fabrication. The analysis and control of the actual grating cross sectional geometry as well as the tilt angle would require further investigation which is beyond the scope of this paper. Nevertheless, the shape of the RCWA theoretical curve reveals that the $T_{2 / 1}$ does not vary very strongly as the $\bar{\mu}$ ratio deviates from the ideal value of 0.5 . An according experimental verification might therefore require the fabrication of gratings with a wider range of $\bar{\mu}$ ratios. We note that the measured $T_{2 / 1}$ ratios for our gratings are considerably lower than what have been achieved with other high-density transmission gratings based on a different fabrication method [143]. Thus, the UV-NIL method used in the fabrication of our gratings appears to deliver a greater accuracy in the space-to-period ratio compared to other methods.

The measured spectra enable another important investigation, which is the comparison of the experimental spectral resolution provided by the grating spectrometer, $\Delta \lambda_{\mathrm{ex}}$, with the theoretically expected resolution, $\Delta \lambda_{\mathrm{th}}$, according to Eq. 6.6. The evaluation of the equation requires some assumption on the diameter, $\Delta S$, of the high-harmonic source area because a direct measurement is difficult. We estimate a source diameter of $\Delta S=35 \mu \mathrm{m}$, using a divergence measurement (beam diameter of $7 \mathrm{~mm}$ at a distance of $R_{s}=9 \mathrm{~m}$ ) and assuming that the high-harmonic radiation propagates as a Gaussian beam. We note that this assumption describes diffraction limited beam propagation and therefore yields a lower limit for the source diameter. Using $\Delta S=35 \mu \mathrm{m}$ and inserting the source and detector distances and the size of the aperture used in our setup $\left(R_{S}=9 \mathrm{~m}, R=120 \mathrm{~mm}\right.$, and $A=100$ 
$\mu \mathrm{m}, p=26 \mu \mathrm{m}$, respectively) we obtain $\Delta \lambda_{t h}=0.09 \mathrm{~nm}$ for the range between 17.5 and $40.5 \mathrm{~nm}$ wavelength.

Retrieving the experimental spectral resolution, $\lambda_{e x}$, from the high-harmonic spectrum in Fig. 6.7(a) requires deconvolving from the spectral width, $w_{\mathrm{q}}$, of a single maximum in Fig. 6.7(a) the spectral bandwidth of the corresponding harmonic radiation, $\Delta \lambda_{q}$. The strongest peak in Fig. 6.7(a), which belongs to the $37^{\text {th }}$ harmonic near $\lambda=21 \mathrm{~nm}$, shows a FWHM of $w_{37}=0.13 \mathrm{~nm}$ based on a Gaussian fit. For determining the smallest possible spectral bandwidth of the $37^{\text {th }}$ harmonic we apply a Fourier argument. The maximum possible duration of emission at a high-harmonic frequency is that of the drive laser pulse, however, the real limit lies at some shorter duration, for instance a factor of 0.7 shorter was observed in [144]. Using the drive laser and focusing parameters given above, with the peak intensity of $2.6 \times 10^{14}$ $\mathrm{W} / \mathrm{cm}^{2}$ and assuming a Gaussian temporal profile for the pulse duration of $39 \mathrm{fs}$ (FWHM), we estimate the duration of the $37^{\text {th }}$ harmonic with the following considerations.

The duration corresponds to the time interval of the drive laser pulse from the earliest moment of emission until phase matching is lost due to a critical level of ionization. The emission starts when the drive leaser reaches the cutoff intensity [34] calculated to be $2.0 \times 10^{14} \mathrm{~W} / \mathrm{cm}^{2}$, which is reached at $11 \mathrm{fs}$ before the peak intensity. It shows that the generation process is not terminated by ionization because the critical ionization fraction [54] of $0.19 \%$ for $\mathrm{Ne}$ gas is not reached in our case (our ADK model calculations [67] yield an ionization of only about $0.05 \%$ ). From this we conclude that the emission of the $37^{\text {th }}$ harmonic terminates only when the drive intensity drops below the cutoff intensity again, which is at $11 \mathrm{fs}$ after peak intensity. This yields an estimated pulse duration of the harmonic, $\tau_{37}=22 \mathrm{fs}$. Assuming a Gaussian pulse shape, we obtain a Fourier limited spectral bandwidth of the $37^{\text {th }}$ harmonic of $\Delta \lambda_{q}=0.03 \mathrm{~nm}$. This is a lower-limit value because any chirp or shorter duration of the harmonic would yield bigger values.

The deconvolution, assuming Gaussian line shapes then yields an upper value for the experimental resolution of $\lambda_{\text {ex }}<0.13 \mathrm{~nm}$. This value compares well with theoretically predicted value of $0.09 \mathrm{~nm}$, when taking into account the remaining uncertainty in the source diameter and $\mathrm{HH}$ radiation bandwidth as named above.

To characterize the spectral resolution of our grating spectrometer also in combination with XUV sources of direct relevance for XUV lithography we used a second source which is based on a Xenon plasma generated by a discharge [145]. The setup is analogous to Fig 1(a), with $\Delta S=570 \mu \mathrm{m}, A=25 \mu \mathrm{m}, p=13.5 \mu \mathrm{m}, R=$ $60 \mathrm{~mm}$ and $R_{s}=1.5 \mathrm{~m}$. Figure 6.8 shows a spectrum of the plasma source measured with our spectrometer over a range from $10 \mathrm{~nm}$ to $17 \mathrm{~nm}$. Compared to the highharmonic source, the spectrum exhibits a higher complexity with partly overlapping lines, but the advantage is the much stronger average output power at $13.5 \mathrm{~nm}$. The 
spectral features that we selected for an evaluation are the two peaks at $16.15 \mathrm{~nm}$ and $16.5 \mathrm{~nm}$ (Xe IX 4d-5p transitions [145]) because these two peaks are almost non-overlapping. Using a decomposition into two Gaussian peaks [see inset in Fig. 6.8], we obtain a FWHM of about $\mathrm{w}_{\mathrm{Xel}}=0.21 \mathrm{~nm}$ for the first peak at $16.15 \mathrm{~nm}$ and $\mathrm{w}_{\text {Xе2 }}=0.24 \mathrm{~nm}$ for the second peak at $16.5 \mathrm{~nm}$. To obtain the experimental spectral resolution, $\lambda_{e x}$, we select the narrower peak, $\mathrm{w}_{\mathrm{Xel}}$ for deconvolution with the spectral bandwidth of the corresponding Xenon radiation, $\Delta \lambda_{X e 1}$. The latter value was obtained from [145] as $\Delta \lambda_{X e 1} \geq 0.13 \mathrm{~nm}$. The deconvolution gives an upper value for the experimental resolution of $\lambda_{\mathrm{ex}}<0.17 \mathrm{~nm}$. For comparison we insert into Eq. 6.6 the experimental parameters and obtain $\Delta \lambda_{\mathrm{th}}=0.11 \mathrm{~nm}$ which is well consistent with the experimental resolution.

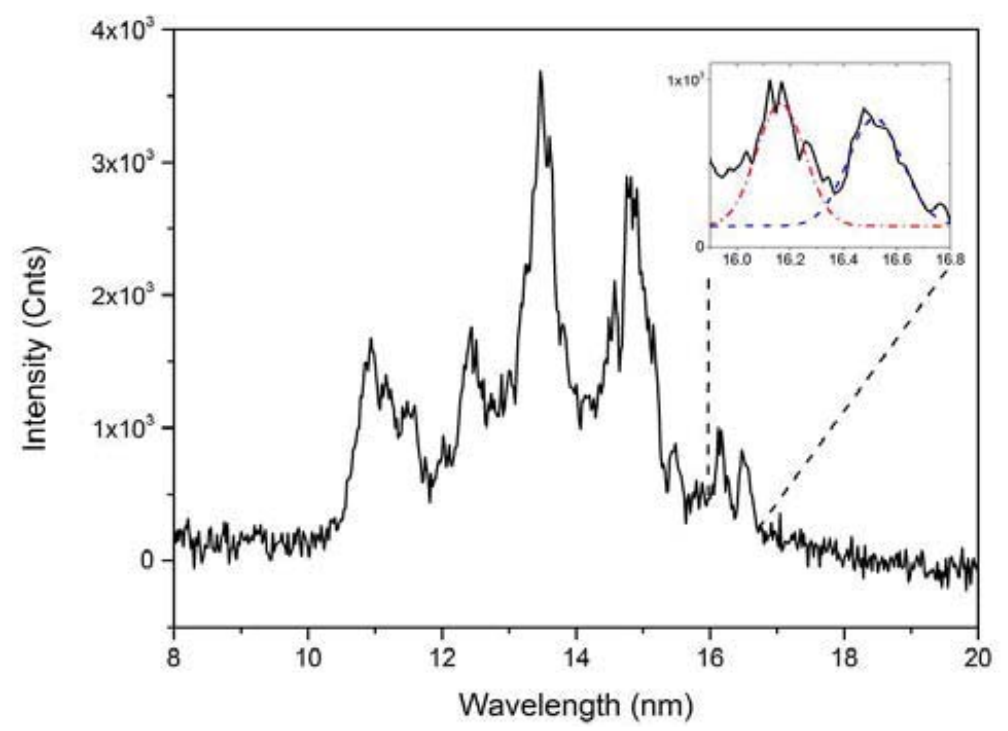

Figure 6.8: Xenon discharge plasma spectrum measured with a 10,000 lines $\mathrm{mm}^{-1}$ grating. The inset in Fig. 6.8 indicates the decomposition of the peaks corresponding to the $4 \mathrm{~d}-5 \mathrm{p}$ transition features for Xenon.

In summary of the resolution measurements, using as an XUV source both HHG and a Xe discharge plasma, we find a spectral resolution that is close to the theoretically possible value. From this we conclude that the UV-NIL based fabrication method demonstrated here indeed provides high-density XUV gratings with superior quality. Furthermore, the XUV source based on HHG has shown to be an excellent source for optical characterization of such gratings for its unique features, namely, the well-separated odd harmonics and the narrow-bandwidth of the individual harmonic order. 


\subsection{Conclusions}

We have fabricated high-line-density transmission gratings for the XUV range using a nano-imprint lithography (NIL) method. The fabrication process is robust and reproducibly delivers high line-density gratings. SEM inspection shows space-toperiod ratios in the range of 0.48 to 0.62 close to the intended ratio of 0.5 and a high degree of reproducibility, in the order of $4 \mathrm{~nm}$ for the width of gratings lines. The quality of the transmission grating was optically characterized by measuring emission spectra from a HHG-based and a plasma-based XUV source. The results show that the measured ratios of the second to first order diffraction efficiency, $T_{2 / 1}$, are as low as desired, in the range of 0.1 to 0.15 , as compared to the almost fourtimes higher values around 0.37 that were reported for other gratings [143]. The optical characterization using the HHG source has shown a grating resolution with an upper-limit of $0.13 \mathrm{~nm}$ which matches the theoretically expected value of 0.09 $\mathrm{nm}$. In summary, the fabrication method applied here proves to be very suitable for providing high quality, high-density transmission gratings for applications in the XUV range. 


\section{Summary and conclusions}

Generating intense radiation in the wavelength band of the extreme ultraviolet (XUV, 100 to $10 \mathrm{~nm}$ ) remains a very challenging goal in today's scientific research. The main reason is due to the lack of suitable laser gain and optical materials that are suitable for the realization of highly reflecting cavity mirrors in that range of short wavelengths. There are a number of fundamentally different approaches to generate radiation in the XUV band, namely, via laser-produced plasma sources (LPP), electron synchrotrons, free-electron lasers (FELs), and via nonlinear optical conversion, specifically high-order harmonic generation (HHG). The suitability of such sources for a particular application depends not only on the range of peak brightness values and available wavelength ranges, but also on other parameters that quantify the quality of the radiation, such as spectral bandwidths, wavelength coverage, wavelength tunability, beam pointing stability, spatial and temporal coherence, pulse-to-pulse stability, repetition rate, divergence and power scalability.

HHG being the subject of the investigation in this thesis gives fully coherent beams in both the temporal and spatial domains. The HHG source is suitable for applications such as lens-less diffractive imaging, where spatially coherent beams are required. In addition, pulse-to-pulse stability is important to have for achieving maximum resolution and optimum utilization of the dynamical range [44] in diffraction imaging. Other examples, where it is of central importance to achieve pulse-to-pulse stability, include increasing the measurement precision for absolute, nonlinear ionization cross sections [58, 59]. Meanwhile, the excellent coherence in both spatial and temporal domain with the possibility of wide wavelength coverage and tunability made HHG source a promising candidate for seeding FELs in order to achieve fully coherent FEL output. The latter is chosen as the prototype application for discussion of the properties of the HHG which is to be well characterized because it shows how stringent a certain properties are required to be. For seeding, it is required not only that the $\mathrm{HH}$ output power is high enough to overcome the FEL shot noise power, but it is also desired that the generated $\mathrm{HH}$ themselves be sufficiently stable from shot to shot and can be tuned for overlapping the gain profile and match the temporal amplification window of an FEL to be injection seeded. It is widely expected that, for increasing the pointing stability and also to provide a longer interaction length, it is promising to make use of waveguiding of the drive laser pulses in a capillary that is filled with the gas to be irradiated. 
We investigated what is to our knowledge the first single-shot analysis of the output beam properties and stability in waveguided high-harmonic generation (HHG). We experimentally characterize the strength of various types of fluctuations in the high-harmonic output, i.e., fluctuations in beam shape, beam pointing, and $\mathrm{HH}$ pulse energy vs. the gas pressure and drive laser pulse energy. The experiments are carried out in a thin waveguiding capillary of standard radius $(75 \mu \mathrm{m}$ inner radius and $37 \mathrm{~mm}$ interaction length) filled with Ar gas, which yields a maximum $\mathrm{HH}$ pulse energy of $1.2 \mathrm{~nJ}$ in the range between $29 \mathrm{~nm}$ and $52 \mathrm{~nm}$ (the $15^{\text {th }}$ and $27^{\text {th }}$ harmonic of a Ti:Sapphire laser) at a drive pulse energy of $1 \mathrm{~mJ}$ at $40 \mathrm{fs}$ drive pulse duration. The maximum available energy for an individual harmonic (the $21^{\text {st }}$ ) is up to $0.4 \mathrm{~nJ}$. At these conditions, we measure the following beam parameters: an energy jitter of $18 \%$, a divergence of $1.5 \mathrm{mrad}(x)$ and $1.6 \mathrm{mrad}(y)$, and a beam pointing fluctuation of $6 \%(x)$ and $17 \%(y)$.

For regular (direct) seeding of a FEL, in which the FEL output wavelength, $\lambda_{\text {FEL, }}$ is the same as the seed laser wavelength, $\lambda_{0}$, the seed power is required to be above approximately a hundred-times [146] the shot noise, $\mathrm{P}_{\mathrm{SN}}$, [146] where $\mathrm{P}_{\mathrm{SN}}$ scales inversely with $\lambda_{0}$. As an example (sFLASH), in ref.[147], $\mathrm{P}_{\text {shot }}$ is calculated to be 10 $\mathrm{W}$ for $\lambda_{0}=13 \mathrm{~nm}$ and therefore seed power of $1 \mathrm{~kW}$ would be required for seeding. To achieve FEL output at shorter wavelength, the scheme named High Gain Harmonic Generation (HGHG) can be applied [30] where seeding is performed at an $\mathrm{n}^{\text {th }}$ sub-harmonic (i.e. longer) wavelength than what the FEL is to generate, $\lambda_{F E L}=$ $\frac{\lambda_{0}}{n}$. However, in this approach, the required seed power becomes much higher, above $100 \times n^{2} \times \mathrm{P}_{\mathrm{SN}}$. So far HGHG as shown to work experimentally with up to the $8^{\text {th }}$ subharmonic $(\mathrm{n}=8)$, to generate an FEL wavelength of $\lambda_{\mathrm{FEL}}=32.5 \mathrm{~nm}$ (FERMI FEL-1) [30] with a seeding wavelength of $\lambda_{0}=260 \mathrm{~nm}$. In this case, a minimum output power of about $60 \mathrm{~kW}$ was required for successful HGHG seeding.

The maximum available energy for individual harmonic $\left(21^{\text {st }}\right)$ is up to 0.4 $\mathrm{nJ} \pm 0.07 \mathrm{~nJ}$, using an estimated pulse duration of $40 \mathrm{fs}$ corresponds to an output power of about $10 \mathrm{~kW} \pm 2 \mathrm{~kW}$. It can be seen that this is yet around a factor of six less than required for HGHG seeding ( $\gtrsim 60 \mathrm{~kW})$. For both regular seeding and HGHG seeding, it is important for the seed beam to overlap transversely with the electron beam. A maximum acceptable offset of $20 \mu \mathrm{m}$ keep the FEL power reduction to below 5\% [62]. The pointing fluctuations of harmonic beams of $6 \%$ $(x)$ and $17 \%(y)$ corresponding to offset of $90 \mu \mathrm{rad}(\mathrm{x})$ and $272 \mu \mathrm{rad}(\mathrm{y}) \operatorname{are}$ however above the maximum acceptable offset.

To investigate the origin of the fluctuations in the harmonic beams, we carried out single-shot correlation measurements where we observed a weak correlation between drive laser pointing or energy fluctuations with pointing or energy fluctuations in the $\mathrm{HH}$ output. We attribute the observed effects to ionizationinduced nonlinear mode mixing and spectral broadening of the drive laser inside the capillary waveguide. Our investigations show that in waveguided HHG it is essential 
to have a very stable drive laser beam with very good beam quality and at the same time apply appropriate pressure and drive laser pulse energy for achieving the best compromise between a high beam stability and a maximum output pulse energy.

For seeding of FELs but also in general, it is desired to achieve higher harmonic output pulse energy without compromising the harmonic beam quality. HHG in standard capillaries, where the diameters are small (typically $150 \mu \mathrm{m}$ diameter) suffers from several fundamental limitations. First, the high-harmonic output pulse energies are limited by ionization-induced phase mismatching when the applied drive laser pulse energy is beyond typically a few hundred $\mu \mathrm{J}$. Second, the drive laser propagation becomes subject to complicated nonlinear propagation effects that may reduce the degree of control over the spectral output properties, and that these effects may turn out to increase the output in certain cases, i.e., via self-compression. The latter shows up as multimodal drive laser propagation and nonlinear mode coupling caused by ionization-induced defocusing. A promising way to circumvent such limitations and still maintain spatial and spectral control would be HHG in a capillary with significantly increased cross section, such that multiple mJ-levels of drive laser pulse energies can be applied without inducing undesired nonlinear pulse propagation dynamics.

Such upscaling of the drive energy has to involve an understanding of the main effects of the experimental parameters on the harmonic output energy, which requires a basic modeling of $\mathrm{HHG}$ in capillaries with increased diameter and a careful characterization of harmonic generation in an experiment. We carried out a theoretical modeling based on spatio-temporal phase-matching which also taking reabsorption of high-harmonics into account, with the model we predict the build-up of high-harmonic pulse energy in an Ar-filled capillary over a wider range of parameters, specifically the drive laser pulse energy, the gas pressure, the capillary diameter and the interaction length. With the model, we calculated the relative yield of high-harmonic energy when we scale up the radius of the capillary with the drive energy.

Experimentally, we generated high-harmonic radiation using an Ar-filled capillary of a large inner diameter $(508 \mu \mathrm{m})$ together with increased drive laser pulse energy, up to $6 \mathrm{~mJ}$. To our knowledge, this is the first experimental demonstration of high-harmonic generation using a waveguide with such a wide inner radius. We characterize the harmonic output as a function of gas pressures and two interaction lengths, $37 \mathrm{~mm}$ and $220 \mathrm{~mm}$. Then we make a comparison between the theoretical and experimental values of the high-harmonic energy as a function of gas pressures for the thick capillary with wide diameter $(508 \mu \mathrm{m})$ using a drive energy of $6.0 \mathrm{~mJ}$, and the thin capillary with standard diameter $(150 \mu \mathrm{m})$ for drive energies in the range of $0.6 \mathrm{~mJ}$ to $1.1 \mathrm{~mJ}$. We find the theoretical values to be matching well with the experimental values for a certain range of lower gas pressure in thick capillaries and lower intensities in the thin capillary. As expected, the developed model can be used to predict parameters that provide a maximum $\mathrm{HH}$ output as long as the degree 
of ionization is kept low such that no multimode propagation of the drive laser within the interaction length has to be taken into account.

We measured a maximum $\mathrm{HH}$ pulse energy of $4 \mathrm{~nJ}$ in the range between $27 \mathrm{~nm}$ and $46 \mathrm{~nm}$ (the $17^{\text {th }}$ and $29^{\text {th }}$ harmonic of a Ti:Sapphire laser) at a drive pulse energy of $6 \mathrm{~mJ}$ at $40 \mathrm{fs}$ drive pulse duration with $37 \mathrm{~mm}$ interaction length. This corresponds to a factor of 3.3 enhancement comparing to using a standard capillary with small inner diameter $(150 \mu \mathrm{m}, 1.2 \mathrm{~nJ})$. The maximum available energy for an individual harmonic (the $21^{\text {st }}$ ) is up to $0.7 \mathrm{~nJ}$. This corresponds to a maximum output power of $17.5 \mathrm{~kW}$ achieved after upscaling. Although HGHG seeding of FEL is still beyond reach, it appears that a wide capillary is better suited for application with higher output requirements, certainly for direct seeding.

It is possible to generate a higher harmonic output in a wide capillary, but in comparison to a standard capillary, it is still open as to what extent the harmonic output can be controlled. An important example of such control is drive laser pulse shaping. For instance, in a wide capillary where the waveguiding induced dispersion is weaker; phase matching occurs at lower gas pressure, which might impose limitations on the spectral control via drive laser shaping because such technique involves a critical timing and sizing of ionization-induced drive laser blue shift. It is therefore important to perform an experimental investigation of the spectral control of HHG in wide-diameter capillaries for identifying the effectiveness of drive laser pulse shaping.

Applying elevated drive energy and using a wide capillary for HHG, with experimental parameters much different from the standard capillary, we have investigated a spectral control of HHG as a function of gas pressure and drive laser pulse duration. The latter is via applying a chirp on the drive laser beam. We found that also, in a wide capillary wavelength tuning of high-harmonic generation is possible. The relative wavelength tuning of the harmonics, $\delta \lambda_{\mathrm{q}} / \lambda_{\mathrm{q}}$, ranged from 0 to -0.0075 via increment in gas pressures and from -0.0025 to +0.015 following the sign of the chirp. We found that the wavelength shift is based not only on ionization induced blueshift as reported for standard capillary, but that other mechanisms (nonadiabatic effects and dependence on drive laser's chirp sign) have a significant contribution as well. The results shows that in spite of rather different operational parameters, the wavelength tuning of high-harmonics in such capillaries can be as effective as in a standard capillary which renders wide-diameter capillary HHG of interest for injection tuning of FELs. Wider tuning might be obtainable with tunable drive laser pulses, such as under investigation as based on parametric amplification [148].

Finally, we demonstrated an application of HHG based on its superior spatial quality and well-known spectral distribution, which is a characterization of nanostructures. We applied the high-harmonic radiation for characterization of freestanding, high-line-density gratings with up to 10,000 lines per $\mathrm{mm}(100 \mathrm{~nm}$ grating period with about $50 \mathrm{~nm}$ wide lines) utilized for an XUV spectrometer. To 
demonstrate the possible advantage of characterization with $\mathrm{HH}$ radiation, the XUV optical properties of the transmission grating were characterized with our HHGbased and a plasma-based XUV source. Using the HHG source, the results showed that the measured ratios of the second to first order diffraction efficiency, $T_{2 / 1}$, are as low as desired, in the range of 0.1 to 0.15 , as compared to the almost four-time higher values around 0.37 that were reported for other gratings. The characterization of the spectral resolution has shown a grating resolution with an upper-limit of 0.13 $\mathrm{nm}$ which matches the theoretically expected value of $0.09 \mathrm{~nm}$. Using a second source which is based on a Xenon plasma generated by a discharge, the optical characterization gives an upper value for the grating resolution of $0.17 \mathrm{~nm}$ which is well consistent with the theoretically expected value of $0.11 \mathrm{~nm}$.

The XUV source based on HHG has shown to be an excellent source for the optical characterization of such gratings for its unique features, namely, the wellseparated odd harmonics and the narrow-bandwidth of the individual harmonic order. Furthermore, the XUV beams for waveguided high-harmonic generation are spatially coherent which is well-suited for determining the diffraction efficiency of the gratings. Compared to the high-harmonic source, the plasma source produces XUV beam with low spatial coherence and its spectrum of exhibits a higher complexity with partly overlapping lines, but the advantage is the much stronger average output power at $13.5 \mathrm{~nm}$ which is of direct relevance for XUV lithography.

In summary, we have thoroughly investigated a number of intrinsic properties of high-harmonic generation in a waveguided geometry. We conclude that using waveguides allows for spatial and spectral control of high-harmonic radiation. Our studies have also shown that waveguided high-harmonic generation is particularly suited for producing radiation at XUV wavelengths with excellent beam coherence and unique spectral features. Future work, focused on using higher drive laser energy and larger waveguide diameters offer the potential of achieving higher XUV output energy. 


\section{Appendix A}

This appendix shows the calculation for the angle of the spherical mirror, $\mathrm{M}_{\mathrm{f}}$, described in Chap.4 which compensates the astigmatism present in the drive laser beam. The drive laser beam is treated as Gaussian beam where its propagation and transformation are calculated based on paraxial ABCD matrix theory. We begin by defining q-parameter and beam parameters for a Gaussian beam. The q-parameter as a function of distance, $\mathrm{z}$, is defined by the following equation:

$$
q(z)=\frac{1}{\frac{1}{R(z)}-i \frac{\lambda}{\pi w(z)^{2}}},
$$

where $R(z)$ is the radius of curvature, given as

$$
R(z)=z\left[1+\frac{z_{R}^{2}}{z^{2}}\right],
$$

where $z_{R}$ is the Rayleigh length, expressed as

$$
z_{R}=\pi w_{0},
$$

and where $w_{0}$ is the beam waist at the position where the radius of curvature is infinity. The evolution of the beam waist during propagation in free space is described by

$$
w(z)=\sqrt{M^{2}} w_{0} \sqrt{1+\frac{z^{2}}{z_{R}{ }^{2}}},
$$

where $M^{2}$ is the M-squared value.

For the calculation that will follow, we first need to determine the q-parameter of our drive laser at the laser exit. In order to do so, we send the drive laser beam through a lens with a focusing length, $f_{\text {lens }}=1000 \mathrm{~mm}$ which was placed at a distance from the laser exit, $z_{\text {laser }}=1070 \mathrm{~mm}$. In horizontal $(\mathrm{x})$ and vertical $(\mathrm{y})$ plane, we determine the distance from the lens to the focus to be $z_{\text {lens } x}=99.0$ $\mathrm{cm} ; z_{\text {lensy }}=101.6 \mathrm{~cm}$ and beam waist to be $w_{0 x}=55 \mu \mathrm{m} ; w_{0 y}=71 \mu \mathrm{m}$. Applying Eq.A3, we obtained the q-parameter at the focus to be 


$$
q_{0}=\left(\begin{array}{l}
i z_{R x} \\
i z_{R y}
\end{array}\right)=\left(\begin{array}{l}
19.80 i \\
11.88 i
\end{array}\right) \mathrm{mm}
$$

To determine the q-parameter of the drive laser beam at the laser exit, we back propagate the drive laser beam. The q-parameter just before the lens is

$$
q_{\text {lens } 1}=\left(\begin{array}{l}
q_{\text {lens } 1 x} \\
q_{\text {lens } 1 y}
\end{array}\right)=q_{0}+\left(\begin{array}{c}
z_{\text {lens }} \\
z_{\text {lensy }}
\end{array}\right)=\left(\begin{array}{r}
20.40+40.25 i \\
-40.22+29.91 i
\end{array}\right) \mathrm{m}
$$

The q-parameter just after the lens is then

$$
q_{\text {lens } 2}=\left(\begin{array}{l}
\frac{q_{\text {lens } 1 x}}{\frac{-q_{\text {lens } 1 x}}{f_{\text {lens }}}+1} \\
\frac{q_{\text {lens } 1 y}}{\frac{-q_{\text {lens } 1 y}}{f_{\text {lens }}}+1}
\end{array}\right)=\left(\begin{array}{r}
19.33+40.24 i \\
-41.29+29.91 i
\end{array}\right) \mathrm{m}
$$

Finally, we obtain the q-parameter at the laser exit to be

$$
q_{\text {exit } 1}=q_{\text {lens } 2}+z_{\text {laser }}=\left(\begin{array}{c}
20.4+40.24 i \\
-40.22+29.91 i
\end{array}\right) \mathrm{m} \text {. }
$$

To propagate the drive laser beam from the laser exit we have to invert the sign for the real part of the q-parameter, thus the q-parameter at the laser exit becomes

$$
q_{\text {exit } 2}=\left(\begin{array}{c}
-20.4+40.24 i \\
40.22+29.91 i
\end{array}\right) \mathrm{m}
$$

Knowing the q-parameter at the laser exit, we can now calculate the tilt angle required by the $\mathrm{M}_{\mathrm{f}}$ mirror to compensate the astigmatism of the drive laser beam at the focus. For mode matching the drive laser beam to the lowest order waveguiding capillary mode, the $M_{f}$ mirror has a radius of curvature of $R_{\text {mirror }}=5 \mathrm{~m}$ and is placed at a distance from the laser exit of $\mathrm{z}_{\text {mirror }}=2 \mathrm{~m}$. The q-parameter of the drive laser beam just before the $\mathrm{M}_{\mathrm{f}}$ mirror is calculated to be

$$
q_{\text {mirror } 1}=\left(\begin{array}{l}
q_{\text {mirror } 1 x} \\
q_{\text {mirror } 1 y}
\end{array}\right)=q_{\text {exit } 2}+z_{\text {mirror }}=\left(\begin{array}{c}
-18.40016+40.24 i \\
-40.22+29.91 i
\end{array}\right) \mathrm{m}
$$

The q-parameter of the drive laser beam just after the $\mathrm{M}_{\mathrm{f}}$ mirror is calculated to be 


$$
q_{\text {mirror } 2}=\left(\begin{array}{c}
\frac{q_{\text {mirror } 1 x}}{\frac{2 \cos \theta q_{\text {mirror } 1 x}}{R_{\text {mirror } 1 x}}+1} \\
\frac{q_{\text {mirror } 1 y}}{\frac{2 q_{\text {mirror } 1 y}}{R_{\text {mirror } 1 y} \cos \theta}+1}
\end{array}\right),
$$

where $\theta$ is the tilt angle of the $\mathrm{M}_{\mathrm{f}}$ mirror. We need to choose $\theta$ such that the real parts of $\mathrm{q}_{\text {mirror2 }}$ are made equal. In this case the two waists (at horizontal and vertical plane) coincide at the same z-position which indicates that the beam is free of astigmatism. At $\theta=14.2$ degree, the q-parameter becomes

$$
q_{\text {mirror } 2}=\left(\begin{array}{c}
-2.51+0.13 i \\
-2.52+0.071 i
\end{array}\right) \mathrm{m}
$$

Thus, we have found the tilt angle of $\mathrm{M}_{\mathrm{f}}$ mirror which compensates the astigmatism in the drive laser beam at the focus to be 14.2 degree. 


\section{References}

1. Maiman, T.H., Stimulated Optical Radiation in Ruby. Nature, 1960. 187(4736): p. 493-494.

2. $\quad$ Pereira, D., et al., A Review of Optically Pumped Far-Infrared Laser Lines from Methanol Isotopes. International Journal of Infrared and Millimeter Waves, 1994. 15(1): p. 1-44.

3. Siegel, P.H., Terahertz Pioneers A Series of Interviews with Significant Contributors to Terahertz Science and Technology. Ieee Transactions on Terahertz Science and Technology, 2015. 5(3): p. 325-325.

4. Daido, H., Review of soft $x$-ray laser researches and developments. Reports on Progress in Physics, 2002. 65(10): p. 1513-1576.

5. $\quad$ Boyd, R.W., Nonlinear Optics2003: Acad. Press.

6. Chapman, H.N., Disruptive photon technologies for chemical dynamics. Faraday Discussions, 2014. 171(0): p. 525-543.

7. Zhavoronkov, N., et al., Microfocus $\mathrm{Cu}$ K-alpha source for femtosecond $x$ ray science. Optics Letters, 2005. 30(13): p. 1737-1739.

8. Jackson, A., Commissioning and Performance of the Advanced LightSource. Proceedings of the 1993 Particle Accelerator Conference, Vols 1-5, 1993: p. 1432-1435.

9. Revol, J.-L., et al., Performance and upgrade of the ESRF light source. Proceedings of IPAC2011, San Sebastián, Spain, 2011: p. THPC009.

10. Feldhaus, J., FLASH-the first soft x-ray free electron laser (FEL) user facility. Journal of Physics B-Atomic Molecular and Optical Physics, 2010. 43(19).

11. Brinkmann, R., The European XFEL Project. Proceedings of FEL 2006, BESY, Berlin, Germany, 2006: p. MOBAU03.

12. Emma, P., et al., First lasing and operation of an angstrom-wavelength freeelectron laser. Nature Photonics, 2010. 4(9): p. 641-647.

13. Young, F.C., Emission of 1-115 Kev Bremsstrahlung from Laser-Produced Plasmas. Bulletin of the American Physical Society, 1976. 21(9): p. 10831083.

14. Sher, M.H., et al., Saturation of the Xe-Iii 109-Nm Laser Using TravelingWave Laser-Produced-Plasma Excitation. Optics Letters, 1987. 12(11): p. 891-893.

15. Nicolosi, P., E. Jannitti, and G. Tondello, Soft-X-Ray Emission of Continua from Laser-Produced Plasmas. Applied Physics B-Photophysics and Laser Chemistry, 1981. 26(2): p. 117-124.

16. Luther, B.M., et al., Saturated high-repetition-rate 18.9-nm tabletop laser in nickellike molybdenum. Optics Letters, 2005. 30(2): p. 165-167. 
17. Jansson, P.A.C., et al., Liquid-tin-jet laser-plasma extreme ultraviolet generation. Applied Physics Letters, 2004. 84(13): p. 2256-2258.

18. Kubiak, G.D., et al., Debris-free EUVL sources based on gas jets. OSA trends in Optics and Photonics, 1996. 4: p. 66-71.

19. Ueno, Y., et al., Debris-Free EUV Source using a through-hole tin target. Proceedings of SPIE Emerging Lithographic Technologies VII 2003. 5037: p. 750-758.

20. Niimi, G., et al., Experimental evaluation of stopping power of high-energy ions from a laser-produced plasma by a magnetic field. Proceedings of the Society of Photo-Optical Instrumentation Engineers 2003. 5037: p. 370-377.

21. Feigl, T., et al., Enhanced reflectivity and stability of high-temperature LPP collector mirrors. Proceedings of Advances in X-Ray/EUV Optics and Components III, 2008. 7077: p. 1-8.

22. Bilderback, D.H., P. Elleaume, and E. Weckert, Review of third and next generation synchrotron light sources. Journal of Physics B-Atomic Molecular and Optical Physics, 2005. 38(9): p. S773-S797.

23. Acremann, Y., et al., Time-resolved imaging of spin transfer switching: Beyond the macrospin concept. Physical Review Letters, 2006. 96(21).

24. Macklin, J.J., et al., Imaging and time-resolved spectroscopy of single molecules at an interface. Science, 1996. 272(5259): p. 255-258.

25. Chapman, H.N., et al., Femtosecond X-ray protein nanocrystallography. Nature, 2011. 470(7332): p. 73-U81.

26. Young, L., et al., Femtosecond electronic response of atoms to ultra-intense $X$-rays. Nature, 2010. 466(7302): p. 56-U66.

27. Glover, T.E., et al., X-ray and optical wave mixing. Nature, 2012. 488(7413): p. 603-608.

28. Kupper, J., et al., X-Ray Diffraction from Isolated and Strongly Aligned Gas-Phase Molecules with a Free-Electron Laser. Physical Review Letters, 2014. 112(8): p. 083002-1-6.

29. Doyuran, A., et al., Characterization of a high-gain harmonic-generation free-electron laser at saturation. Physical Review Letters, 2001. 86(26): p. 5902-5905.

30. Allaria, E., et al., Highly coherent and stable pulses from the FERMI seeded free-electron laser in the extreme ultraviolet. Nature Photonics, 2012. 6(10): p. 699-704.

31. Mcpherson, A., et al., Studies of Multiphoton Production of Vacuum Ultraviolet-Radiation in the Rare-Gases. Journal of the Optical Society of America B-Optical Physics, 1987. 4(4): p. 595-601.

32. Ferray, M., et al., Multiple-Harmonic Conversion of 1064-Nm Radiation in Rare-Gases. Journal of Physics B-Atomic Molecular and Optical Physics, 1988. 21(3): p. L31-L35.

33. Bloembergen, N., Nonlinear optics1996: World Scientific. 
34. Corkum, P.B., Plasma Perspective on Strong-Field Multiphoton Ionization. Physical Review Letters, 1993. 71(13): p. 1994-1997.

35. Paul, P.M., et al., Observation of a train of attosecond pulses from high harmonic generation. Science, 2001. 292(5522): p. 1689-1692.

36. Mairesse, Y., et al., Attosecond synchronization of high-harmonic soft xrays. Science, 2003. 302(5650): p. 1540-1543.

37. Corkum, P.B. and F. Krausz, Attosecond science. Nature Physics, 2007. 3(6): p. 381-387.

38. Feng, X.M., et al., Generation of Isolated Attosecond Pulses with 20 to 28 Femtosecond Lasers. Physical Review Letters, 2009. 103(18): p. 183901-14.

39. Sola, I.J., et al., Controlling attosecond electron dynamics by phasestabilized polarization gating. Nature Physics, 2006. 2(5): p. 319-322.

40. Fohlisch, A., et al., Direct observation of electron dynamics in the attosecond domain. Nature, 2005. 436(7049): p. 373-376.

41. Niikura, H., et al., Sub-laser-cycle electron pulses for probing molecular dynamics. Nature, 2002. 417(6892): p. 917-922.

42. Antoine, P., A. LHuillier, and M. Lewenstein, Attosecond pulse trains using high-order harmonics. Physical Review Letters, 1996. 77(7): p. 1234-1237.

43. Bellini, M., et al., Temporal coherence of ultrashort high-order harmonic pulses. Physical Review Letters, 1998. 81(2): p. 297-300.

44. Sandberg, R.L., et al., Lensless diffractive imaging using tabletop coherent high-harmonic soft-x-ray beams. Physical Review Letters, 2007. 99(9).

45. Balcou, P., et al., High-Order Harmonic-Generation in Rare-Gases - a New Source in Photoionization Spectroscopy. Zeitschrift Fur Physik D-Atoms Molecules and Clusters, 1995. 34(2): p. 107-110.

46. Larsson, J., et al., 2-Color Time-Resolved Spectroscopy of Helium Using High-Order Harmonics. Journal of Physics B-Atomic Molecular and Optical Physics, 1995. 28(3): p. L53-L58.

47. Descamps, D., et al., Extreme ultraviolet interferometry measurements with high-order harmonics. Optics Letters, 2000. 25(2): p. 135-137.

48. Froud, C.A., et al., Soft-x-ray wavelength shift induced by ionization effects in a capillary. Optics Letters, 2006. 31(3): p. 374-376.

49. Reitze, D.H., et al., Enhancement of high-order harmonic generation at tuned wavelengths through adaptive control. Optics Letters, 2004. 29(1): p. 86-88.

50. Maltezopoulos, T., et al., A high-harmonic generation source for seeding a free-electron laser at $38 \mathrm{~nm}$. Applied Physics B-Lasers and Optics, 2014. 115(1): p. 45-54.

51. Lee, J., D.R. Carlson, and R.J. Jones, Optimizing intracavity high harmonic generation for XUV fs frequency combs. Opt Express, 2011. 19(23): p. 23315-23326. 
52. Ravasio, A., et al., Single-Shot Diffractive Imaging with a Table-Top Femtosecond Soft X-Ray Laser-Harmonics Source. Physical Review Letters, 2009. 103(2): p. 028104-1-5.

53. Takahashi, E., et al., Generation of highly coherent submicrojoule soft $x$ rays by high-order harmonics. Physical Review A, 2002. 66(2): p. 021802$1-4$.

54. Durfee, C.G., et al., Phase matching of high-order harmonics in hollow waveguides. Physical Review Letters, 1999. 83(11): p. 2187-2190.

55. Popmintchev, T., et al., Phase matching of high harmonic generation in the soft and hard X-ray regions of the spectrum. Proceedings of the National Academy of Sciences of the United States of America, 2009. 106(26): p. 10516-10521.

56. Tamaki, Y., et al., Phase-matched high-order-harmonic generation in a gasfilled hollow fiber. Physical Review A, 1999. 59(5): p. 4041-4044.

57. Butcher, T.J., et al., Bright extreme-ultraviolet high-order-harmonic radiation from optimized pulse compression in short hollow waveguides. Physical Review A, 2013. 87(4): p. 43822.

58. Hasegawa, H., et al., Multiphoton ionization of He by using intense highorder harmonics in the soft-x-ray region. Physical Review A, 2005. 71(2): p. 023407-1-5.

59. Nabekawa, Y., et al., Production of doubly charged helium ions by twophoton absorption of an intense sub-10-fs soft $x$-ray pulse at $42 \mathrm{eV}$ photon energy. Physical Review Letters, 2005. 94(4): p. 043001-1-4.

60. Labat, M., et al., High-Gain Harmonic-Generation Free-Electron Laser Seeded by Harmonics Generated in Gas. Physical Review Letters, 2011. 107(22): p. 224801-1-5.

61. Togashi, T., et al., Extreme ultraviolet free electron laser seeded with highorder harmonic of Ti:sapphire laser. Optics Express, 2011. 19(1): p. 317324.

62. Azima, A., et al., Tolerance studies on the high harmonic laser seeding at FLASH Proceedings of FEL 2008 p. 235.

63. Spitzenpfeil, R., et al., Enhancing the brilliance of high-harmonic generation. Applied Physics A, 2009. 96(1): p. 69-81.

64. L.V.Keldysh, Ionization in the field of a strong electromagnetic wave. Soviet Physics Jetp-Ussr, 1965. 20(1307).

65. Lompre, L.A., et al., High-Order Harmonic-Generation in Xenon - Intensity and Propagation Effects. Journal of the Optical Society of America BOptical Physics, 1990. 7(5): p. 754-761.

66. L'Huillier, A., K.J. Schafer, and K.C. Kulander, Higher-order harmonic generation in xenon at $1064 \mathrm{~nm}$ : The role of phase matching. Physical Review Letters, 1991. 66(17): p. 2200-2203. 
67. M.V. Ammosov, N.B.D., and V.P. Krainov, Tunnel ionization of complex atoms and of atomic ions in an alternating electromagnetic field. Soviet Physics Jetp-Ussr, 1986. 64(6): p. 1191.

68. Augst, S., et al., Laser Ionization of Noble-Gases by Coulomb-Barrier Suppression. Journal of the Optical Society of America B-Optical Physics, 1991. 8(4): p. 858-867.

69. Krause, J.L., K.J. Schafer, and K.C. Kulander, High-Order HarmonicGeneration from Atoms and Ions in the High-Intensity Regime. Physical Review Letters, 1992. 68(24): p. 3535-3538.

70. Lhuillier, A., et al., High-Order Harmonic-Generation Cutoff. Physical Review A, 1993. 48(5): p. R3433-R3436.

71. Budil, K.S., et al., Influence of Ellipticity on Harmonic-Generation. Physical Review A, 1993. 48(5): p. R3437-R3440.

72. Gaarde, M.B., et al., Spatiotemporal separation of high harmonic radiation into two quantum path components. Physical Review A, 1999. 59(2): p. 1367-1373.

73. Salieres, P., A. Lhuillier, and M. Lewenstein, Coherence Control of HighOrder Harmonics. Physical Review Letters, 1995. 74(19): p. 3776-3779.

74. Lewenstein, M., P. Salieres, and A. Lhuillier, Phase of the Atomic Polarization in High-Order Harmonic-Generation. Physical Review A, 1995. 52(6): p. 4747-4754.

75. Chang, Z., et al., Temporal phase control of soft-x-ray harmonic emission. Physical Review A, 1998. 58(1): p. R30-R33.

76. Marcatili, E.A.J. and R.A. Schmeltzer, Hollow Metallic Dielectric Waveguides for Long Distance Optical Transmission Lasers. Bell System Technical Journal, 1964. 43(4p2): p. 1783-1809.

77. Nubling, R.K. and J.A. Harrington, Launch conditions and mode coupling in hollow-glass waveguides. Optical Engineering, 1998. 37(9): p. 2454-2458.

78. Wahlstrom, C.G., et al., High-Order Harmonic-Generation in Rare-Gases with an Intense Short-Pulse Laser. Physical Review A, 1993. 48(6): p. 4709-4720.

79. Kazamias, S., et al., Global optimization of high harmonic generation. Physical Review Letters, 2003. 90(19): p. 193901.

80. Sutherland, J.R., et al., High harmonic generation in a semi-infinite gas cell. Opt Express, 2004. 12(19): p. 4430-4436.

81. Pfeifer, T., et al., Spatial control of high-harmonic generation in hollow fibers. Optics Letters, 2005. 30(12): p. 1497-1499.

82. Powell, F.R., et al., Thin-Film Filter Performance for Extreme Ultraviolet and X-Ray Applications. Optical Engineering, 1990. 29(6): p. 614-624.

83. Sommerer, G., H. Rottke, and W. Sandner, Enhanced efficiency in highorder harmonic generation using sub-50-fs laser pulses. Laser Physics, 1999. 9(1): p. 430-432. 
84. Rudawski, P., et al., A high-flux high-order harmonic source. Review of Scientific Instruments, 2013. 84(7): p. 073103-1-7.

85. Burge, R.E., et al., Time dependence of the spatial coherence of the 23.6and 23.2-nm radiation from the germanium soft-x-ray laser. Journal of the Optical Society of America B-Optical Physics, 1998. 15(5): p. 1620-1626.

86. Bartels, R.A., et al., Generation of spatially coherent light at extreme ultraviolet wavelengths. Science, 2002. 297(5580): p. 376-378.

87. Erny, C., et al., Metrology of high-order harmonics for free-electron laser seeding. New Journal of Physics, 2011. 13: p. 073035-1-20.

88. Leitner, T., et al., Shot-to-shot and average absolute photon flux measurements of a femtosecond laser high-order harmonic photon source. New Journal of Physics, 2011. 13: p. 093003-1-11.

89. Nisoli, M., et al., Effects of carrier-envelope phase differences of fewoptical-cycle light pulses in single-shot high-order-harmonic spectra. Physical Review Letters, 2003. 91(21): p. 213905-1-4.

90. Schnurer, M., et al., Guiding and high-harmonic generation of sub-10-fs pulses in hollow-core fibers at 10(15) W/cm(2). Applied Physics B-Lasers and Optics, 1998. 67(2): p. 263-266.

91. Pfeifer, T., et al., Controlling the spectral shape of coherent soft X-rays. Applied Physics B-Lasers and Optics, 2005. 80(3): p. 277-280.

92. Bartels, R., et al., Shaped-pulse optimization of coherent emission of highharmonic soft X-rays. Nature, 2000. 406(6792): p. 164-166.

93. Ardana-Lamas, F., et al., Spectral characterization of fully phase-matched high harmonics generated in a hollow waveguide for free-electron laser seeding. New Journal of Physics, 2013. 15: p. 073040-1-10.

94. Wagner, N.L., et al., Self-compression of ultrashort pulses through ionization-induced spatiotemporal reshaping. Physical Review Letters, 2004. 93(17): p. 173902-1-4.

95. Anderson, P.N., et al., High-energy laser-pulse self-compression in short gas-filled fibers. Physical Review A, 2014. 89(1): p. 013819-1-10.

96. Geskus, D., Design \& Construction of Regenerative Amplifier and Compressor for Chirped Pulse Amplification. M.Sc. dissertation, University of Twente, Dept. of Science \& Technology, Laser Physics \& Non-Linear Optics, , 2006.

97. Siegman, A.E., Lasers1986: University Science Books.

98. Goh, S.J., et al., Fabrication and characterization of free-standing, highline-density transmission gratings for the vacuum UV to soft $X$-ray range. Opt Express, 2015. 23(4): p. 4421-4434.

99. Flettner, A., et al., Ellipticity dependence of atomic and molecular high harmonic generation. European Physical Journal D, 2002. 21(1): p. 115119.

100. The Center for X-Ray Optics website: [ www.cxro.lbl.gov ]. 
101. Rae, S.C., Ionization-Induced Defocusing of Intense Laser-Pulses in HighPressure Gases. Optics Communications, 1993. 97(1-2): p. 25-28.

102. Skobelev, S.A., et al., Ionization self-compression of intense femtosecond pulses propagating through gas-filled dielectric capillaries. Jetp Letters, 2009. 89(11): p. 540-546.

103. Rundquist, A., et al., Phase-matched generation of coherent soft X-rays. Science, 1998. 280(5368): p. 1412-1415.

104. Constant, E., et al., Optimizing high harmonic generation in absorbing gases: Model and experiment. Physical Review Letters, 1999. 82(8): p. 1668-1671.

105. Brichta, J.P., et al., Comparison and real-time monitoring of high-order harmonic generation in different sources. Physical Review A, 2009. 79(3).

106. Encyclopedia of Measurement and Statistics Thousand Oaks, CA: SAGE Publications, Inc.

107. Bartels, R., et al., Attosecond time-scale feedback control of coherent X-ray generation. Chemical Physics, 2001. 267(1-3): p. 277-289.

108. Eden, J.G., High-order harmonic generation and other intense optical fieldmatter interactions: review of recent experimental and theoretical advances. Progress in Quantum Electronics, 2004. 28(3-4): p. 197-246.

109. Bellini, M., C. Corsi, and M.C. Gambino, Neutral depletion and beam defocusing in harmonic generation from strongly ionized media. Physical Review A, 2001. 64(2): p. 023411-1-10.

110. T. J. Butcher, e.a., Bright extreme-ultraviolet high-order-harmonic radiation from optimized pulse compression in short hollow waveguides. Physical Review A, 2013. 87(4): p. p. 43822-1-4.

111. Ditmire, T., et al., Spatial coherence measurement of soft $x$-ray radiation produced by high order harmonic generation. Physical Review Letters, 1996. 77(23): p. 4756-4759.

112. Zhang, X.S., et al., Quantum-path control in high-order harmonic generation at high photon energies. New Journal of Physics, 2008. 10: p. 025021-1-9.

113. Lhuillier, A., K.J. Schafer, and K.C. Kulander, Theoretical Aspects of Intense Field Harmonic-Generation. Journal of Physics B-Atomic Molecular and Optical Physics, 1991. 24(15): p. 3315-3341.

114. Lhuillier, A., K.J. Schafer, and K.C. Kulander, High-Order HarmonicGeneration in Xenon at 1064-Nm - the Role of Phase Matching. Physical Review Letters, 1991. 66(17): p. 2200-2203.

115. Zhang, J., Z.H. Lu, and L.J. Wang, Precision refractive index measurements of air, N-2, O-2, Ar, and CO2 with a frequency comb. Appl Opt, 2008. 47(17): p. 3143-3151.

116. National Institute of Standards and Technology website: [http://physics.nist.gov/ffast]. 
117. Rogers, E.T.F., et al., Spatiotemporal phase-matching in capillary highharmonic generation. Journal of the Optical Society of America B-Optical Physics, 2012. 29(4): p. 806-812.

118. Chapman, R.T., et al., Modal effects on pump-pulse propagation in an Arfilled capillary. Opt Express, 2010. 18(12): p. 13279-13284.

119. Goh, S.J., et al., Single-shot fluctuations in waveguided high-harmonic generation. Opt Express, 2015. 23(19): p. 24888-902.

120. Tournois, P., Acousto-optic programmable dispersive filter for adaptive compensation of group delay time dispersion in laser systems. Optics Communications, 1997. 140(4-6): p. 245-249.

121. Wang, G.L., et al., Influence of gas pressure on high-order-harmonic generation of Ar and Ne. Physical Review A, 2011. 84(5): p. 053404-1-9.

122. Shin, H.J., et al., Nonadiabatic blueshift of high-order harmonics from $\mathrm{Ar}$ and Ne atoms in an intense femtosecond laser field. Physical Review A, 2001. 63(5): p. 053407-1-9.

123. Chen, M.C., et al., Bright, coherent, ultrafast soft X-ray harmonics spanning the water window from a tabletop light source. Phys Rev Lett, 2010. 105(17): p. 173901.

124. Zhou, J., et al., Enhanced high-harmonic generation using $25 f_{s}$ laser pulses. Physical Review Letters, 1996. 76(5): p. 752-755.

125. Carlisle, J.A., et al., Probing the Graphite Band-Structure with Resonant Soft-X-Ray Fluorescence. Physical Review Letters, 1995. 74(7): p. 12341237.

126. Wagner, C. and N. Harned, EUV LITHOGRAPHY Lithography gets extreme. Nature Photonics, 2010. 4(1): p. 24-26.

127. Bakshi, V., EUV Sources for Lithography2006: Society of Photo Optical.

128. Ullrich, J., A. Rudenko, and R. Moshammer, Free-Electron Lasers: New Avenues in Molecular Physics and Photochemistry. Annual Review of Physical Chemistry, Vol 63, 2012. 63: p. 635-660.

129. Wilhelm, K., et al., Observations of the sun at vacuum-ultraviolet wavelengths from space. Part I: Concepts and instrumentation. Space Science Reviews, 2004. 111(3-4): p. 415-480.

130. de Bruijn, R., et al., Absorption of EUV in laser plasmas generated on xenon gas jets. Journal of Quantitative Spectroscopy \& Radiative Transfer, 2003. 81(1-4): p. 97-105.

131. Word, M.J., I. Adesida, and P.R. Berger, Nanometer-period gratings in hydrogen silsesquioxane fabricated by electron beam lithography. Journal of Vacuum Science \& Technology B, 2003. 21(6): p. L12-L15.

132. Savas, T.A., et al., Large-area achromatic interferometric lithography for $100 \mathrm{~nm}$ period gratings and grids. Journal of Vacuum Science \& Technology B, 1996. 14(6): p. 4167-4170. 
133. McMullin, D.R., et al., Extreme-ultraviolet efficiency measurements of freestanding transmission gratings. Appl Opt, 2004. 43(19): p. 3797-3801.

134. Vratzov, B., et al., Large scale ultraviolet-based nanoimprint lithography. Journal of Vacuum Science \& Technology B, 2003. 21(6): p. 2760-2764.

135. Schnopper, H.W., et al., Diffraction Grating Transmission Efficiencies for Xuv and Soft X-Rays. Appl Opt, 1977. 16(4): p. 1088-1091.

136. Born, M. and E. Wolf, Principles of optics : electromagnetic theory of propagation, interference and diffraction of light. 6th ed1980, Oxford; New York: Pergamon Press. xxvii, 808 p.

137. Sailaja, S., et al., A simple XUV transmission grating spectrograph with subangstrom resolution for laser-plasma interaction studies. Measurement Science \& Technology, 1998. 9(9): p. 1462-1468.

138. Palmer, C., Diffraction Grating Handbook. 6th ed: Newport Corporation). Chap. 8.

139. RSoft DiffractMOD, "RSoft Design Group." http://www.rsoftdesign.com.

140. Ceglio, N.M., et al., Demonstration of Guided-Wave Phenomena at Extreme-Ultraviolet and Soft-X-Ray Wavelengths. Optics Letters, 1988. 13(4): p. 267-269.

141. Markert, T.H., et al., Modeling the diffraction efficiencies of the AXAF high energy transmission gratings. Euv, X-Ray, and Gamma-Ray Instrumentation for Astronomy Vi, 1995. 2518: p. 424-437.

142. Reichardt, G. and F. Schafers, Laminar versus trapezoidal grating profiles: AFM-measurements and efficiency simulations. Gratings and Grating Monochromators for Synchrotron Radiation, 1997. 3150: p. 121-129.

143. Desaute, P., et al., Characterization of a high resolution transmission grating. Optics Communications, 2000. 173(1-6): p. 37-43.

144. Bouhal, A., et al., Cross-correlation measurement of femtosecond noncollinear high-order harmonics. Journal of the Optical Society of America B-Optical Physics, 1997. 14(4): p. 950-956.

145. Kieft, E.R., et al., Time-resolved pinhole camera imaging and extreme ultraviolet spectrometry on a hollow cathode discharge in xenon. Physical Review E, 2003. 68(5): p. 056403.

146. Reiche, S., Overview of seeding methods for FELs.

147. Lambert, G., et al., An optimized $\mathrm{kHz}$ two-colour high harmonic source for seeding free-electron lasers and plasma-based soft x-ray lasers. New Journal of Physics, 2009. 11: p. 083033-1-17.

148. Hoppner, H., et al., An optical parametric chirped-pulse amplifier for seeding high repetition rate free-electron lasers. New Journal of Physics, 2015. 17: p. 053020-1-12. 


\section{List of publications}

\section{Journals}

S.J. Goh, H.J.M. Bastiaens, B. Vratzov, Q. Huang, F. Bijkerk, and K.J. Boller, Fabrication and characterization of free-standing, high-line-density transmission gratings for the vacuum UV to soft X-ray range, Optics Express, 23(4): p. 4421$4434(2015)$

S.J. Goh, Y. Tao, P.J.M van der Slot, H.J.M. Bastiaens, S. G. Biedron, M.B. Danailov, S.V. Milton, J. Herek, K. J. Boller, Single-shot fluctuations in waveguided high-harmonic generation, Optics Express, 23(19): p. 24888-24902 (2015)

S.J. Goh, Y. Tao, P.J.M van der Slot, H.J.M. Bastiaens, S. G. Biedron, M.B. Danailov, S.V. Milton, J. Herek, K. J. Boller, Spectral control of high-harmonic generation via drive laser shaping in a wide-diameter capillary, in preparation

\section{Conference proceedings}

S.J. Goh, Y. Tao, P.J.M van der Slot, H.J.M. Bastiaens, S. G. Biedron, M.B. Danailov, S.V. Milton, J. Herek, K. J. Boller, High-harmonic source for seeding of FERMI@Elettra, Proceedings of FEL 2010, Malmö, Sweden, TUPB04: p.262-265 (2010)

\section{Oral presentations}

S.J. Goh, J. Reinink, Y. Tao, P.J.M van der Slot, H.J.M. Bastiaens, S. G. Biedron, M.B. Danailov, S.V. Milton, J. Herek, K. J. Boller, Coherent control of highharmonic generation in a large-volume capillary for seeding of free-electron lasers, Oral presentation, $26^{\text {th }}$ Symposium Plasma Physics \& Radiation Technology, Lunteren, The Netherlands, 12-13 March 2014

S.J. Goh, Y. Tao, P.J.M van der Slot, H.J.M. Bastiaens, S. G. Biedron, M.B. Danailov, S.V. Milton, J. Herek, K. J. Boller, High-harmonic generation in a largevolume capillary for seeding of free-electron lasers, Oral presentation, Conference on High Intensity Laser and Attosecond Science in Israel (CHILI), Tel-Aviv, Israel, 2-4 December 2013

S.J. Goh, Y. Tao, P.J.M van der Slot, H.J.M. Bastiaens, S. G. Biedron, M.B. Danailov, S.V. Milton, J. Herek, K. J. Boller, Characterization of high-harmonic beam generated in a gas-filled capillary for seeding of free-electron lasers, Oral presentation, Deutsche Physikalische Gesellschaft Frühjahrstagung (DPGFrühjahrstagung Sektion AMOP), Hannover, 18-22 March 2013 


\section{Poster presentations}

S.J. Goh, J. Reinink, Y. Tao, P.J.M van der Slot, H.J.M. Bastiaens, S. G. Biedron, M.B. Danailov, S.V. Milton, J. Herek, K. J. Boller, Scaling of high-harmonic generation in a gas-filled capillary, Poster presentation, $37^{\text {th }}$ Annual Meeting of the Division Atomic, Molecular and Optical Physics (AMO), Lunteren, The Netherlands, 1-2 October 2013

S.J. Goh, Y. Tao, P.J.M van der Slot, H.J.M. Bastiaens, S. G. Biedron, M.B. Danailov, S.V. Milton, J. Herek, K. J. Boller, Characterization of a gas-filled capillary-based high-harmonic source for seeding of free-electron lasers, Poster presentation, 25th Symposium Plasma Physics \& Radiation Technology, Lunteren, 5-6 March 2013

S.J. Goh, Y. Tao, P.J.M van der Slot, H.J.M. Bastiaens, S. G. Biedron, M.B. Danailov, S.V. Milton, J. Herek, K. J. Boller, Characterization of high-harmonic beams for seeding of free-electron lasers, Poster presentation, 36th Annual Meeting of the Division Atomic, Molecular and Optical Physics (AMO), Lunteren, The Netherlands, 9-10 October 2012

S.J. Goh, Y. Tao, P.J.M van der Slot, H.J.M. Bastiaens, S. G. Biedron, M.B. Danailov, S.V. Milton, J. Herek, K. J. Boller, Stability study of high-harmonic generation in a capillary for seeding of free-electron lasers, Poster presentation, $35^{\text {th }}$ Annual Meeting of the Division Atomic Molecular and Optical Physics (AMO), Lunteren, The Netherlands, 11-12 October 2011

S.J. Goh, Y. Tao, P.J.M van der Slot, H.J.M. Bastiaens, S. G. Biedron, M.B. Danailov, S.V. Milton, J. Herek, K. J. Boller, A tunable, soft x-ray high order harmonic source for seeding of free-electron lasers, Poster presentation, $23^{\text {rd }} \mathrm{NNV}$ Plasma Physics \& Radiation Technology Symposium, Lunteren, The Netherlands, 15-16 March 2011

S.J. Goh, Y. Tao, P.J.M van der Slot, H.J.M. Bastiaens, S. G. Biedron, M.B. Danailov, S.V. Milton, J. Herek, K. J. Boller, High-harmonic sources for seeding of FERMI@Elettra, Poster presentation,Physics@FOM Veldhoven 2011, 18-19 January 2011

S.J. Goh, Y. Tao, P.J.M van der Slot, H.J.M. Bastiaens, S. G. Biedron, M.B. Danailov, S.V. Milton, J. Herek, K. J. Boller, High-harmonic Generation in GasFilled Capillary Waveguide for seeding of FERMI@Elettra, Poster presentation, Mesa+ Annual Meeting 2010, 14 September, 2010 


\section{Acknowledgments}

This Ph.D. thesis is accomplished not solely on my own, but through the efforts and support of many people I met over these years. Here, I would like to express my sincere gratitude to them. First, I like to recognize Klaus Boller, my promotor, for his continuous support in my Ph.D. research and for his patience, motivation, and immense knowledge. Next, I would like to express my deep gratitude to my copromotor, Bert Bastiaens for his guidance in the laboratory as well as the help I received in many other aspects of my Ph.D. research. I also like to extend my gratitude to Peter van der Slot for his guidance and advice on various subjects encountered during my research.

I like to thank all the other members of Laser Physics and Nonlinear Optics group, Caterina, Chris, Jonathon, Jörn, Kasper, Marco, Muharrem, Ruud, Robert, Thomas, Tom, Yin, Youwen and Ingrid. I have had a nice time with you all during the coffee breaks and group outings. Further thanks go to Gerard, Johan and Fred for the help I received in the lab. I also like to extend my appreciation to the members of the COPS group, for lending me lab equipment and Dan from OS group, for sharing his laser time with me in the beginning of my Ph.D.

I would like to thank Boris and QiuShi from XUV Industrial Focus Group for our collaboration in the publication of transmission grating paper. And a special thanks is extended to Miltcho for his help in testing the breakdown of my laser over the workshop in FERMI@Elettra. To my graduation committee members, Helmut Zacharias, Jennifer Herek, Fred Bijker and Carsten Fallnich, thank you for reading my thesis and providing me valuable feedback. Further thanks go to Simon for proofreading my thesis.

The next people I like to thank are the close friends I made here, Rong, Lei Lei, Altaf, Yolanda, Nan and Tawfiq. You guys have been great companies and I will always cherish our friendship. I also like to thank my besties back home, Meng Yee, Pooi Gee and Saw Hoon for your encouragement this whole time, xoxo! Last but not least, I am thankful to my family members who have been supportive in my pursuit of the Ph.D. I hope I have made you all proud! 